\title{
Abstracts from the 2017 American College of Medical Toxicology (ACMT) Annual Scientific Meeting
}

\begin{abstract}
These are the abstracts of the 2017 American College of Medical Toxicology (ACMT) Annual Scientific Meeting. Included here are 120 abstracts that will be presented in March 2017, including research studies from around the globe and the ToxIC collaboration, clinically significant case reports describing new toxicologic phenomena, and encore presentations from other scientific meetings.
\end{abstract}

\begin{abstract}
Keywords Abstracts · Annual Scientific Meeting · Toxicology Investigators Consortium · Medical Toxicology Foundation · Pediatric Environmental Health Specialty Units
\end{abstract}

Correspondence American College of Medical Toxicology (ACMT) 10645 N. Tatum Blvd Phoenix, AZ info@acmt.net

\section{Introduction}

The American College of Medical Toxicology (ACMT) received 144 eligible research abstracts for consideration for presentation at the 2017 Annual Scientific Meeting (ASM), including 72 research studies and 72 case reports. Each abstract was reviewed in a blinded fashion by at least four medical toxicologists. Each abstract was independently scored based on the clinical question, data source, analytic method, results/conclusion, and clarity of presentation. A total of 120 abstracts were accepted, for an overall acceptance rate of $83 \%$. The acceptance rate was $98 \%$ for research studies and $68 \%$ for case reports. This work would not be possible without the hard work and diligence of our abstract reviewers: Vik Bebarta, Katie Boyle, Diane Calello, Stephanie Carreiro, Jon Cole, Kirk Cumpston, Kristin Engebretsen, Yaron Finkelstein, David Jang, Louise Kao, Ken Katz, Russ Kerns, Eric Lavonas, Michael Levine, Gerry Maloney, Mark Mycyk, Anne Riederer, Dan Rusyniak, Sam Stellpflug, Richard Wang, Brandon Wills, and Luke Yip. Even more important is the contribution of the ACMT staff. Lizzy Nguyen led the process, with significant assistance from Tara Frutkin, Tricia Steffey, and Paul Wax. Congratulations to all the researchers whose work will be presented in Puerto Rico. The 2017 Annual Scientific Meeting promises to be fantastic. We look forward to seeing you there.

Sam Stellpflug, MD, FACMT, Abstract Review Chair

Russ Kerns, MD, FACMT, Chair, ACMT Research Committee

\section{Original Research: Platform Sessions}

\section{A Novel Oral Fluid Assay for the Detection of Fentanyl After Suspected Heroin Overdose}

Matthew Griswold ${ }^{1}$, Peter Chai ${ }^{1}$, Brittany Chapman ${ }^{1}$, Melissa Friscia ${ }^{2}$, Alexander Krotulski ${ }^{2}$, Edward Boyer ${ }^{1}$, Barry Logan ${ }^{2}$, Kavita Babu ${ }^{1}$

${ }^{1}$ University of Massachusetts School of Medicine, Worcester, MA, USA

${ }^{2}$ Center for Forensic Science Research and Education, Willow Grove, PA, USA

Background: The adulteration of heroin with clandestine fentanyl is one suspected etiology for the striking increase in heroin-related overdose deaths. While drug testing in urine is both validated and commonplace, oral fluid testing is preferable in specific environments (e.g., methadone clinics). In this study, we compared an oral fluid swab with gold standard urine testing for the detection of fentanyl in individuals presenting after heroin overdose.

Hypothesis: We hypothesized that detection of fentanyl in oral fluid would be equivalent to validated urine drug testing methods.

Methods: Adult heroin users presenting to an urban, tertiary care ED for care after overdose were eligible for enrollment if they had received naloxone (bystander or EMS) and were able to provide verbal consent. After consent, subjects were interviewed about pharmaceutical fentanyl use, and a urine specimen was collected. Next, a Quantisal swab (Immunalysis; Pomona, CA) was placed in the subject's mouth until indicator changed or $10 \mathrm{~min}$ elapsed. Samples were kept refrigerated, then shipped to NMS Labs for analysis via Liquid Chromatography Time-Of-Flight Mass Spectrometry. This study was approved by the Institutional Review Board.

Results: Between 09/01/16 and 11/04/16, 20 adult heroin users who presented after overdose were enrolled in a convenience sample. Average age was $31 ; 50 \%$ participants identified as female. All 20 urine samples were identified as containing fentanyl, while 18/20 oral fluid samples were positive for fentanyl, indicating a $90 \%$ agreement rate. Acetylfentanyl was identified in $4 / 20$ oral swab specimens, but only 2 corresponding urine specimens.

Discussion: Participants successfully tolerated the oral swabs. For the detection of fentanyl, oral fluid and urine results agreed in $90 \%$ of paired specimens. Acetylfentanyl was identified in $4 / 20$ cases (20\%) using oral swabs, but only paired $2 / 20$ cases by urine drug testing. Time since exposure and persistence in urine might contribute to cases in which oral fluid and urine testing failed to agree. Limitations of this study include the small sample size.

Conclusion: Bedside oral fluid swabs offer a feasible and accurate method for detection of clandestine fentanyls. Further study is required to determine the sensitivity and specificity of this methodology.

\section{Mitochondrial Dysfunction in Patients With Carbon Monoxide} Poisoning Treated With Hyperbaric Oxygen

David Jang, Matthew Kelly, David Lambert, Kevin Hardy, Frances Shofer, David Eckmann

University of Pennsylvania, Philadelphia, PA, USA

Background: Carbon monoxide (CO) poisoning is the leading cause of poisoning mortality and morbidity in the United States. Carboxyhemoglogin $(\mathrm{COHb})$ levels are not predictive of severity or prognosis of exposure. At this time, the measurement of mitochondrial respiration may serve as a potential biomarker in $\mathrm{CO}$ poisoning and treatment. This is a preliminary study enrolling patients with confirmed $\mathrm{CO}$ poisoning treated with hyperbaric oxygen (HBO).

Methods: With informed consent, we prospectively studied 6 patients $>18$ years of age having confirmed carbon monoxide exposure treated with $\mathrm{HBO}$ and 6 volunteers undergoing $\mathrm{HBO}$ as controls. A single venous blood sample was collected from each subject before and after $\mathrm{HBO}$. Peripheral blood mononuclear cells (PBMCs) were placed in a 2-mL 
chamber at a final concentration of 3-4 $310^{6}$ cells $/ \mathrm{mL}$. Measurement of oxygen consumption were performed at $37{ }^{\circ} \mathrm{C}$ in a high-resolution oxygraph (Oxygraph-2k Oroboros Instruments, Innsbruck, Austria). Oxygen flux (in pmol $\mathrm{O}_{2} / \mathrm{s} / 10^{6}$ cells), which is directly proportional to oxygen consumption, was recorded continuously using DatLab software 6. Basal, ATP-linked, residual oxygen consumption (ROX), and maximal mitochondrial oxygen consumption rates along with specific complexlinked activity (in pmol/s/10 $/ 10^{6}$ cells) were measured in permeabilized PBMCs using a standard series of substrates, inhibitor, and uncoupler injections. Cell isolation and all metabolic measurements were performed within $2 \mathrm{~h}$.

Results: The range of $\mathrm{COHb}$ was $15-62 \%$ for patients with $\mathrm{CO}$ poisoning. There were differences in respiration before and after $\mathrm{HBO}$ in the $\mathrm{CO}$ group with a general increase in respiration: routine respiration $9.2 \mathrm{pmol}$ $\mathrm{O}_{2} / \mathrm{s} / 10^{6}$ cells; $95 \%$ CI 6.1 to $13.2, p<0.0001$; proton leak $0.83 \mathrm{pmol} \mathrm{O}_{2} /$ $\mathrm{s} / 10^{6}$ cells; $95 \% \mathrm{CI}-0.23$ to $1.9, p=0.157$; maximal respiration $26.7 \mathrm{pmol} \mathrm{O}_{2} / \mathrm{s} / 10^{6}$ cells; $95 \%$ CI 18.3 to $28.5, p<0.0001$; $\mathrm{ROX}$ $0.22 \mathrm{pmol} \mathrm{O}_{2} / \mathrm{s} / 10^{6}$ cells; $95 \% \mathrm{CI}-0.58$ to $1.31, p=0.557$. There were no differences in the control group before and after HBO.

Conclusions: The key parameters of mitochondrial respiration were significantly lower in CO patients when compared to the control group with a significant increase in key parameters of mitochondrial respiration after HBO. This pilot clinical study demonstrates that HBO treatment may result in improvement in mitochondrial respiration.

\section{Acute Adverse Events Associated With The Administration of FabAV Within The North American Snakebite Registry}

Kurt Kleinschmidt $^{1}$, Anne-Michelle Ruha ${ }^{2}$, Paul Wax ${ }^{1,4}$, Jeffrey Brent ${ }^{3}$, Sharan Campleman ${ }^{4}$, On Behalf of the Toxicology Investigators Consortium NASBR

${ }^{1}$ University of Texas Southwestern Medical Center, Dallas, TX, USA

${ }^{2}$ Center for Toxicology and Pharmacology Education and Research,

University of Arizona College of Medicine, Phoenix, AZ, USA

${ }^{3}$ University of Colorado, School of Medicine, Aurora, CO, USA

${ }^{4}$ American College of Medical Toxicology, Phoenix, AZ, USA

Background: Most data relative to hypersensitivity-related, acute adverse events (AEs) associated with FabAV (BTG International Inc., West Conshohocken, PA; $\mathrm{CroFab}($ ) $)$ are retrospective. Prospective data are limited. The prevalence of treatment approaches to FabAV-related AEs is also poorly described. ACMT's North American Snakebite Registry (NASBR) is a prospective database established in 2013. One component collected by the NASBR is FabAV-related data on AE's.

Research Questions: The primary objective is to characterize the prevalence of hypersensitivity-related, acute AEs associated with FabAV administration. A secondary objective is to describe the treatment used for these AEs.

Methods: All cases prospectively reported to the NASBR between 1/ 2013 and 12/2015 were reviewed. Descriptive statistics were used.

Results: Three hundred sixty-eight patients received $\geq 1$ vial of FabAV. Among these, $149(40.2 \%)$ were children $<18$ years of age. AEs occurred in five $(2.3 \%)$ of the adults and in four $(2.7 \%)$ of the children $(2.4 \%$ total). The five different adults totaled $13 \mathrm{AEs}$ and the four different children totaled eight AEs. The most common AEs for adults occurred at $0.9 \%$ each for three different AEs: rash, hypotension, and bronchospasm. The most common AE for children was rash. Eight of nine with an $\mathrm{AE}$ received specific treatment consisting of various combinations of antihistamines $(N=6)$, steroids $(N=2)$, epinephrine $(N=2)$, slowed infusion $(N=2)$, or stopped infusion $(N=1)$. Fifteen of the 368 patients received prophylaxis medications prior to FabAV administration; none had an AE.

Discussion: Previously published AE prevalence with FabAV has varied much. In three prospective studies, eight (14\%) of 57 patients had AEs. In retrospective series, there are 480 adult patients of whom $22(4.6 \%)$ had AEs, and there are 118 children patients of whom 7 (5.9\%) had AEs (combined-598 total patients with 29 AEs [4.8\%]). Our 2.4\% AE prevalence is lower than that reported in previous prospective or retrospective studies. Patients responded well to treatments of the AEs and only one patient had the FabAV infusion stopped completely. Serum sickness was excluded.

Conclusion: Our large prospective study indicates that acute hypersensitivity AEs occurred in only $2.4 \%$ of patients, which is less frequent than in prior studies.

\section{This research was performed in collab- oration with the ACMT Toxicology Investigators Consortium. \\ Toxicology Investigators CONSORTIUM}

\section{The Epidemiologic and Clinical Characteristics of Snakebites in the North American Snakebite Registry}

Anne-Michelle Ruha ${ }^{1}$, Kurt Kleinschmidt ${ }^{2}$, Spencer Greene ${ }^{3}$, Meghan B Spyres $^{4}$, Paul Wax ${ }^{2}$, Jeffrey Brent ${ }^{5}$, Angela Padilla-Jones ${ }^{1}$, Sharan Campleman ${ }^{6}$, On Behalf of the Toxicology Investigators Consortium NASBR

${ }^{I}$ Center for Toxicology and Pharmacology Education and Research, University of Arizona College of Medicine - Phoenix, Phoenix, Arzona, USA

${ }^{2}$ University of Texas Southwestern Medical Center, Dallas, Texas, USA

${ }^{3}$ Baylor College of Medicine, Houston, Texas, USA

${ }^{4}$ University of Southern California, Los Angeles, California, USA

${ }^{5}$ University of Colorado, Aurora, Colorado, USA

${ }^{6}$ American College of Medical Toxicology, Phoenix, Arizona, USA

Background: ACMT established the North American Snakebite Registry (NASBR) in 2013 to enhance knowledge of snake envenomation in humans.

Research Question: What are the epidemiologic and clinical characteristics of snakebites in the NASBR?

Methods: All cases prospectively reported to the NASBR between 1/ 2013 and 12/2015 were reviewed. Descriptive statistics were used.

Results: Fourteen sites from 10 states contributed 450 cases. Ninetynine percent were due to native species and $>99 \%$ of these pit vipers. Rattlesnakes accounted for $58 \%$, copperheads $29 \%$, cottonmouths $3 \%$, and unidentified pit vipers $9 \%$. Sixty-nine percent of bites occurred in men and $42 \%$ in children $\leq 18$ years. Lower extremities were affected in $54 \%$, but the most common site was a finger (32\%). Intentional interactions led to $19 \%$ of bites. Native pit viper-related $(n=442)$ local tissue effects included swelling in $96 \%$, ecchymosis in $62 \%$, and erythema in $39 \%$. Systemic effects were vomiting (before opioids) in $7.2 \%$, minor bleeding in $6.3 \%$, and neurotoxicity in $5.2 \%$. Hypotension occurred in 15 patients $(3.4 \%)$ and 2 were intubated. Hematologic effects included thrombocytopenia $(10.6 \%)$, hypofibrinogenemia $(11.8 \%)$, and coagulopathy (14.0\%). Treatment included CroFab ${ }^{\circledR}$ antivenom in $84.7 \%$ of patients with a pit viper bite. Maintenance doses were used in $30.3 \%$ of these. $\geq 1$ dose of antibiotics was given to 34 patients $(7.7 \%)$, with 2 reported confirmed infections. Debridement of bullae accounted for $69 \%$ of procedures. There were 6 fasciotomies, with 2 having elevated intracompartmental pressure documented. Length of stay was $<48 \mathrm{~h}$ in $78.3 \%$. Fourteen patients were readmitted after discharge, with 8 reporting late hematologic toxicity (1 with late bleeding). Six were retreated with antivenom. See Tables for more details.

Discussion: The NASBR is a national registry of detailed case information regarding snakebite. Four hundred fifty cases were amassed in 3 years. General demographics were similar to those reported in national poison and injury databases. However, more detailed and informative 
clinical information that may guide public health interventions and management of this condition was gained. The NASBR is a powerful tool for the study of snake envenomation.

\section{This research was performed in collab- (2) X oration with the ACMT Toxicology \\ Toxicology Investigators Investigators Consortium.}

\section{Prognostic Utility of Initial Lactate for ED Drug Overdose Fatality: A Validation Cohort \\ Randy Cheung ${ }^{1}$, Robert Hoffman ${ }^{2}$, David Vlahov ${ }^{3}$, Alex Manini ${ }^{4}$ \\ ${ }^{1}$ State University of New York, Buffalo, NY, USA \\ ${ }^{2}$ NYU School of Medicine, New York, NY, USA \\ ${ }^{3}$ University of California, San Francisco, CA, USA \\ ${ }^{4}$ Icahn School of Medicine at Mount Sinai, New York, NY, USA}

Background: We previously demonstrated that the initial ED lactate had prognostic utility for in-hospital mortality from acute drug poisoning.

Research Question: To validate the prognostic utility of initial lactate for drug overdose fatality in ED patients.

Methods: This was an observational, prospective, cohort study over 5 years at two urban teaching hospitals. Subjects were consecutive adult (>18 years) ED acute drug overdose patients; we excluded children, prehospital cardiac arrest, alternative diagnoses, non-drug overdose, and missing data. Demographics, history, vitals, and drug exposures were obtained from medical records using standardized data abstraction. Initial lactate was drawn as part of clinical care by ED clinicians; the primary outcome was inpatient fatality, and the secondary outcome was occurrence of shock (vasopressor requirement). Receiver operating characteristics (ROC) were plotted using SPSSv22 to determine optimal lactate cutpoint (point that maximizes sensitivity + specificity), along with test characteristics (sensitivity/specificity), area under the curve (AUC), odds ratios (OR), and 95\% confidence intervals (CI).

Results: Out of 3739 patients screened, 2333 met exclusion criteria (1487 missing lactate, 376 children, 278 missing outcomes, 141 alternate diagnoses, 37 non-drugs, 14 prehospital arrests), leaving 1406 patients for analysis (56\% female, mean age 43.1 years), of whom 54 patients had shock (3.9\%) and 24 died $(1.7 \%)$. Mean initial lactate $(\mathrm{mmol} / \mathrm{L})$ was $8.1 \pm 5.6$ for fatalities and $2.4 \pm 6.7$ for survivors $(p<0.001)$. The AUC for prediction of fatality was 0.85 (CI $0.73-0.95$ ). The optimal lactate cutpoint for fatality was $5.0 \mathrm{mmol} / \mathrm{L}$ (OR 34.2, CI 13.7-84.2, 70.8\% sensitive, 93.3\% specific) and the occurrence of either shock or death was $2.7 \mathrm{mmol} / \mathrm{L}$ (OR 7.9, CI 4.5-13.9). Initial lactate under $2.0 \mathrm{mmol} / \mathrm{L}$ had $99.5 \%$ negative predictive value (CI 98.8-99.9). Drug classes for which initial lactate had the highest utility for prediction of fatality were as follows: salicylates (AUC $=0.98$, cutpoint $=6.0)$, sympathomimetics $($ AUC $=0.98$, cutpoint $=7.8)$, acetaminophen $(\mathrm{AUC}=0.98$, cutpoint $=10.0)$, opioids $(\mathrm{AUC}=0.97$, cutpoint $=3.1)$, digoxin $(\mathrm{AUC}=0.92$, cutpoint $=2.4)$, anti-convulsants (AUC $=0.91$, cutpoint $=3.0$ ); lactate had lowest utility for beta-/Ca-channel blockers $(\mathrm{AUC}=0.73$, cutpoint $=7.1)$, diuretics $(\mathrm{AUC}=0.55$, cutpoint $=1.1$, and ACE inhibitors $(\mathrm{AUC}=0.16$, cutpoint $=0.9)$.

Discussion: The highest prognostic utility was for salicylates, sympathomimetics, and acetaminophen.

Conclusion: Lactate should be used as a biomarker for early decisionmaking in ED patients with acute drug overdose.

\section{Utilizing a Machine Based Learning Algorithm to Predict Mortality in Acetaminophen Overdose}

Nathan Menke ${ }^{2}$, Anthony Pizon ${ }^{1}$, Marie Menke ${ }^{1}$

${ }^{T} U P M C$, Pittsburgh, PA, USA

${ }^{2}$ VAPHS, Pittsburgh, PA, USA
Background: The decision to transplant in cases of acetaminophen (APAP)-induced acute liver failure (ALF) requires early, accurate identification of patients with the highest likelihood of mortality. The accuracy of current clinical decision tools is limited.

Hypothesis: We hypothesize that a machine based learning algorithm (MBLA) will more accurately identify the clinical outcomes of APAPinduced ALF patients as compared to the most commonly utilized clinical decision tool (Kings College Criteria (KCC)).

Methods: Our study is a retrospective cohort. We compared the accuracy of a MBLA (support vector machine) and the KCC to predict clinical outcomes; patients were classified as survivors or non-survivors. The study sample is comprised of a cohort of patients admitted between July 2004 and June 2014 to the transplant ICU at an academic, tertiary care center. Study variables $(\mathrm{pH}, \mathrm{PT}$, creatinine, and encephalopathy stage) were obtained at two time points: (1) time of admission and (2) $24 \mathrm{~h}$. Inclusion criteria were APAP toxicity and ALF (defined as acute liver injury and coagulopathy) within 1 week of the overdose. Exclusion criteria were history of previous kidney/renal disease, prisoner, pregnant, or patient received a transplant. Statistical analysis was performed using $\mathrm{R}$ version 2.12.1.

Results: Complete cases were found for 40 patients (16 deaths). The KCC, at arrival, classified $26 / 40(65 \%$; $95 \%$ CI $48-79 \%)$ cases correctly. The KCC, at $24 \mathrm{~h}$, classified 29/40 (73\%; 95\% CI of 48-79\%) cases correctly. At $24 \mathrm{~h}$, the MBLA classified the outcome of 39/40 (98\%; $95 \%$ CI of $85-100 \%$ ) cases correctly (one death was misidentified as a survivor). The MBLA was significantly more accurate than the KCC at arrival and $24 \mathrm{~h}$ ( $p$ values $<0.001$ and 0.004 , respectively).

Discussion: The MBLA significantly outperformed the KCC in predicting the outcomes of patients that developed ALF from APAP. The ability to accurately identify patients at high risk for mortality at $24 \mathrm{~h}$ would allow earlier listing for transplantation. A larger, independent data set is required to validate the results of this study.

Conclusion: MBLAs may provide a useful tool to assist clinical decisionmaking for determining the need for hepatic transplantation in cases of APAP toxicity.

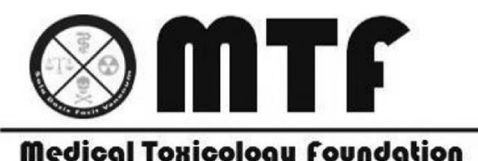

This research was supported by the 2014 MTF Innovative Research and Teaching Award.

medical Toricology foundation

7. Carbon Monoxide Concentrations Induced by Cigarette Smoking Outdoors in Typical Smokers

N George, J Schimmel, S Suner, JB Hack

Brown University / Rhode Island Hospital, Providence, RI, USA

Background: Non-invasive screening of carboxyhemoglobin saturation (SpCO) in the emergency department (ED) to detect occult exposures is increasingly common. The threshold for non-toxic SpCO levels in nonsmokers is $<2 \%$ and for smokers up to $9 \%$. The literature supporting this baseline SpCO among smokers is inadequate, and the impact of active smoking on SpCO concentration is unclear.

Research Question: The primary objective was to characterize baseline $\mathrm{SpCO}$ in a cohort of typical smokers in outdoor public spaces. Secondary objectives were to explore how SpCO changes during and after smoking and to compare SpCO levels between smokers and non-smokers.

Methods: This was a prospective cohort study in two outdoor urban areas in the United States, in a convenience sample of adult smokers enrolled consecutively. SpCO concentrations were assessed non-invasively before, during, and after cigarette smoking with a pulse $\mathrm{CO}$ oximeter. Analyses include descriptive statistics, two-sample $t$ test, sensitivity analysis, and correlations. 
Results: Eighty-five smokers had mean $\mathrm{SpCO}$ concentrations before, during, and after smoking of 2.7 (SD 2.6), 2.7 (SD 2.5), and 3.1 (SD 2.9), respectively. Fifteen controls had SpCO 1.3 (SD 1.3). There was a significant difference in baseline SpCO between the two groups of 1.4 (95\% CI for difference $0.6-2.2 ; P<0.01, t$ test). There was correlation among individual smokers' SpCO levels before and after smoking ( $r$ 0.8; $P<0.01$, Pearson coefficient), but no clear trend. There was no relationship between time since last cigarette and $\mathrm{SpCO}$ (P $=$ NS, Pearson coefficient). Only $20 \%$ of smokers reached an $\mathrm{SpCO}>5 \%$.

Discussion: Cigarette smokers had a mean peak SpCO of $3.1 \%$-far below the generally accepted "normal" of up to $9 \%$. In contrast to the expected pattern, there was not a significant trend in SpCO during active smoking.

Conclusion: Our findings suggest that while some smokers have $\mathrm{SpCO}$ concentrations $>5 \%$, most do not. Elevated levels in smokers should not routinely be accepted as normal and $\mathrm{CO}$ exposure from another source should be considered.

\section{Toxicology Consultation - Successful Reimbursement for Telemedicine Services}

Timothy Wiegand, Peter Crane, Ken Conner, Mike Kamali, Rosali Wratni, Tracey Loveland, Ron Montante, Marilyn Reif

University of Rochester Medical Center, Rochester, NY, USA

Background: Providing patient care through telemedicine is a costeffective way of performing toxicology consultation when direct bedside care is not feasible. It is primarily performed using interactive audio and video telecommunication, which facilitates real-time interaction between the toxicologist and the patient. While telemedicine has been utilized informally for years in various types of medical practices, it has been only recently that opportunities for billing and reimbursement have become available. On January 1, 2016, the New York Telehealth Coverage Law prohibited commercial insurers from excluding telehealth services from coverage when the service would otherwise have been covered if delivered in-person. This effectively allowed for telemedicine billing and reimbursement in New York State, although not all insurance carriers immediately adopted payment and reimbursement practices.

Research Question: What is the reimbursement profile of medical toxicology consultations performed via telemedicine?

Methods: Retrospective review of billing records related to medical toxicology consultations performed using telemedicine. Consultations took place after approval of the New York Telehealth Coverage Law on 1/1/ 2016 over a 4-month period and occurred when bedside consultation was not feasible. Telehealth consultation was performed using secure accounts on password-protected tablet computers utilizing Zoom Meeting® platform for connection that allowed direct audio/video conferencing between the medical toxicologist and patient. The primary purpose of our telemedicine consultations was for clearance purposes or to assess and provide recommendations regarding diagnosis and treatment or to instruct on antidote use.

Results: Sixteen toxicology encounters utilizing three different CPT codes for initial inpatient telehealth consultation were performed. Specific CPT codes included G0425 (Focused) 30 min (\#13), G0426 (Detailed) $50 \mathrm{~min}$ (\#2), and G0427 (Comprehensive) 70 min (\#1). 9/16 $(56 \%)$ of the encounters were successfully billed. Reimbursement ranged from $\$ 42.73$ to $\$ 217.72$ with average of $\$ 105.22$ and totaled $\$ 939.39$ overall.

Conclusion: This data describes the first successfully billed telehealth consultations in medical toxicology. Although there is variation of coverage and reimbursement by state, telemedicine encounters represent an opportunity to enhance productivity, improve efficiency, and generate revenue from toxicology practice while continuing to provide excellent patient care for toxicologic encounters.
9. Pseudoephedrine to Methamphetamine: Comparison of Percent Conversion between Abuse-Deterrent and Non Abuse-Deterrent Formulations

Brandon Presley $^{1}$, Bob Bianchi ${ }^{2}$, John Coleman ${ }^{3}$, Fran Diamond ${ }^{1}$, Gerry McNally ${ }^{4}$.

${ }^{1}$ NMS Labs, Willow Grove, PA, USA

${ }^{2}$ Prescription Drug Research Center, Bradenton FL, USA

${ }^{3}$ Prescription Drug Research Center LLC, Clifton, VA, USA

${ }^{4}$ Johnson \& Johnson Consumer Inc., Fort Washington, PA, USA

Background: Clandestine chemists ("cooks") have demonstrated the ability to convert commercially available pseudoephedrine (PSE) to methamphetamine. Some PSE formulations have abuse-deterrent properties that manufacturers claim limit or prevent conversion to methamphetamine. These studies evaluated commercially available PSE products for ease of extraction and conversion to methamphetamine using fundamental "kitchen chemistry" techniques.

Methods: These experimental methodology studies compared 2 marketed PSE products with abuse-deterrent properties (PSE-A and PSE-B) to a comparator product without abuse-deterrent properties (PSE-C). Extractability was evaluated using 8 readily available tools, 2 common solvents, 2 extraction temperatures, and unstirred versus stirred reaction conditions. Eight solvents were compared for their ability to extract PSE from manipulated, solubilized tablets. The one-pot conversion method commonly used in clandestine settings was slightly modified to determine impact on methamphetamine yield. Quantities and addition times of the chemicals used were varied; reaction duration was varied; the procedure was scaled down and conducted in a controlled environment to reduce risk. Chemicals and equipment were purchased from local retail stores. PSE and methamphetamine were quantified using high performance liquid chromatography-tandem mass spectrometry (HPLC-MS/ MS). Standard calculations determined quantities of PSE and methamphetamine recovered and converted, respectively. Raw data including the calculated means and percentages are reported.

Results: A rotating blade coffee grinder effectively reduced particle size. Dissolution rates of ground tablets and physical characteristics varied by product; PSE-A was most resistant to dissolution while PSE-B and PSE-C were comparable and able to be dissolved completely, demonstrating no dissolution-deterrent properties. Conditions of the one-pot conversion method were modified to increase the yield of methamphetamine. The modified process resulted in $43-47 \%$ conversion of extracted PSE-A, 46 $60 \%$ of extracted PSE-B, and 49-66\% of extracted PSE-C.

Discussion: Experimental conditions resulting in conversion of approximately $50 \%$ of abuse-deterrent and non-abuse deterrent PSE products to methamphetamine were identified using a slightly modified standard clandestine process.

Conclusion: This series of methodological evaluations confirmed that "cooks" can produce methamphetamine readily and with a yield that is similar from PSE products with and without abuse-deterrent technology.

10. Role of Lipid Emulsion in Management of Organophosphate Compound Poisoning

Ashish Bhalla, Bharath Chhabria, Nusrat Shafiq, Susheel Kumar, Navneet Sharma

PGIMER, Chandigarh, India

Objectives: To prospectively study the effects of $20 \%$ lipid emulsion (Intralipid) morbidity and mortality in organophosphate compound (OPC) poisoning.

Methodology: A prospective open label pilot study was carried out at a tertiary care hospital in north India. Forty patients were enrolled and managed with conventional treatment. None of the patients were given 2 PAM. In addition, $100 \mathrm{ml}$ of $20 \%$ intralipid was administered intravenously at admission. Patients were monitored till discharge or death. During the stay, 
vitals, hematologic and biochemical parameters, morbidity (duration of hospital stay, duration of ventilation), and mortality were noted. The results were compared with age and sex-matched historic controls.

Results: The cases comprised of $57.5 \%$ males and $42.5 \%$ females, with a mean age of 30.9 years. Baseline characteristics were comparable between the intervention and the control groups. The respiratory symptoms improved early and there was a statistically significant difference between cases and controls at 24 and $48 \mathrm{~h}(p=0.011,0.01$ chi-square). There was no change in hemodynamic parameters between the groups after the intervention ( $p=\mathrm{NS} t$ test). While both groups showed an equal incidence of fever initially, temperatures normalized earlier in the cases compared to controls ( $p=0.03$, chi-square). There was no significant change from baseline in hemodynamic, hematological, and biochemical profiles after administration of intralipid in intervention group. The complication rates were equal in both groups ( $p=\mathrm{NS}$, chi-square) except for ventilatorassociated pneumonia which was higher in historic controls $(p=0.04$, chi-square). The mean duration of mechanical ventilation was 1.9 days in cases versus 6.3 days in controls ( $p=0.001, t$ test) The duration of hospital stay was also markedly reduced in the intervention group with a mean of 4.8 days versus 10.63 days in controls ( $p=0.00, t$ test).

Conclusion: A significant reduction in morbidity (duration of hospital stay, mechanical ventilation) was noted in the intervention arm; however, there was no significant change in mortality. Comparison with historic controls was a significant limitation of this study.

\section{A New Drug Epidemic: Emergency Department Visits for Synthetic Cannabinoid (SC) Intoxication With Laboratory Analysis of SC Samples}

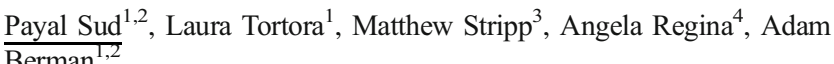

${ }^{1}$ Long Island Jewish Medical Center, Northwell Health, New Hyde Park, NY, USA

${ }^{2}$ North Shore University Hospital, Northwell Health, Manhasset, NY, USA ${ }^{3}$ Carolinas Medical Center, Carolinas Poison Center, Charlotte, NC, USA

${ }^{4}$ St. Barnabas, Bronx, NY, USA

Objectives: Use of synthetic cannabinoids (SC) has emerged as a new drug epidemic. Our emergency departments (EDs) received a surge of SC users presenting with lethargy and bradycardia, contrasting prior reports of SC-induced tachycardia and agitation. We aim to describe these novel presentations and characterize the compounds.

Methods: We present a case series of patients with SC intoxication who presented to our toxicology service covering two tertiary care EDs. A retrospective chart review recorded initial vital signs, chief complaint, and clinical course. Urine, blood, and xenobiotic samples were analyzed using gas chromatography/mass spectrometry. Spectra were compared against the SWGDRUG database and scored based on a reference comparison. Urine samples were compared to an expanded SC panel using liquid chromatography/mass spectrometry.

Results: Between 2/11/2015 and 6/23/2015, 141 visits were identified. Males comprised 139 visits (age range 21-68 years; median 35, interquartile range 20). Sixty-eight percent presented with lethargy or loss of consciousness. Hypotension (SBP $<90 \mathrm{mmHg}$ ) and bradycardia (HR $<60 \mathrm{bpm}$ ) were seen in 10 and $24 \%$ of visits, respectively. While most patients were discharged after observation, 3 were admitted to the ICU and 7 to telemetry. Admissions were for vital sign instability, bradycardia requiring pacing, prolonged sedation, and respiratory failure requiring mechanical ventilation. Laboratory analysis revealed SC in the XLR-11 family in 19/36 drug, 9/12 blood, and 23/31 urine samples. INACA family compounds were detected in 23/36 drug samples, 13/31 urine samples, but no blood samples. Others detected included PB-22 and several 5-fluorinated compounds. No JWH compounds, opiates, imidazoline receptor agonists, benzodiazepines, or other sedativehypnotics were detected.
Conclusion: Unlike their predecessors, novel SC may be associated with significant CNS depression and bradycardia. While prior reports indicated that SC mostly contained JWH compounds, none were detected in these samples. The most commonly identified compounds in this series were in the XLR-11 and INACA families. These tend to be full agonists at the cannabinoid receptor and are presumably more potent. The lack of other depressants suggests that the clinical findings are due to the combination of these compounds and not coingestants. SC intoxication should be considered for patients with undifferentiated psychomotor depression and bradycardia.

\section{Citalopram Ingestions: a Two-Year Retrospective Study of the Toxicology Investigators Consortium}

Tony Rianprakaisang ${ }^{1}$, Lynn Farrugia ${ }^{2}$, Sean Rhyee ${ }^{3}$, On Behalf of the Toxicology Investigators Consortium (ToxIC)

${ }^{1}$ University of Connecticut School of Medicine, Farmington, CT, USA

${ }^{2}$ Hartford Hospital, Hartford, CT, USA

${ }^{3}$ Saint Francis Hospital and Medical Center, Hartford, CT, USA

Introduction: The selective serotonin reuptake inhibitors (SSRIs) have an improved safety profile compared to older antidepressants (e.g., tricyclic antidepressants), though adverse effects including seizures can occur in overdose. Among the SSRIs, seizure risk is reportedly higher with citalopram (up to $13.1 \%$ ), even compared to escitalopram, the therapeutically active S-enantiomer. The Toxicology Investigators Consortium (ToxIC) database collects cases of toxicological exposures as reported by Medical Toxicologists who have evaluated patients at the bedside. Our study uses this database to further investigate the rate of seizures and risk factors for seizures after citalopram ingestion.

Methods: The ToxIC database was queried from January 1, 2013 through December 31, 2015. Cases involving citalopram exposure were considered for inclusion. Exclusion criteria were any cases involving co-ingestion of escitalopram, bupropion, or tramadol (the latter two agents are well known to also cause seizures). The primary outcome measured was incidence of seizures. Additional demographic and clinical features were analyzed for any increased association with the primary outcome: gender, tachycardia (HR >140), bradycardia (HR <50), hypertension (SBP >200), hypotension (SBP <80), QTc prolongation (QTc $>500 \mathrm{~ms}$ ), CNS depression, delirium, and neuromuscular excitability (e.g., tremor, myoclonus, hyperreflexia).

Results: Three hundred thirteen cases were identified, with 274 cases remaining after application of exclusion criteria. Seizures occurred in 36 cases $(13.1 \%)$. There was a trend towards higher seizure risk in women, but it was not statistically significant (OR $1.54,95 \%$ CI $0.69-3.41)$. Of the clinical features analyzed, the following had a significantly increased association with seizures: tachycardia (OR 2.65, 95\% CI 1.21-5.82) and QTc prolongation (OR 3.83, 95\% CI 1.66-8.85). The remaining clinical features did not carry an increased association with seizures: bradycardia (OR 2.24, 95\% CI 0.45-11.24), hypotension (OR 2.60, 95\% CI 0.89 27.59), hypertension (OR 1.07, 95\% CI 0.05-21.2), CNS depression (OR $0.89,95 \%$ CI $0.41-1.92$ ), delirium (OR $0.54,95 \%$ CI $0.12-2.36$ ), and neuromuscular excitability (OR $1.30,95 \%$ CI $0.58-2.92$ ).

Discussion: In this study, the overall incidence of seizures with citalopram was similar to prior studies. Patients with tachycardia and QTc prolongation had a significantly higher rate of seizures. Further investigation is required to further clarify additional risk factors, such as other co-ingestants.

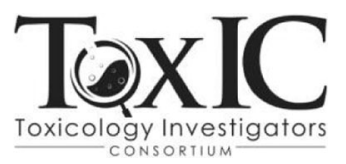

This research was performed in collaboration with the ACMT Toxicology Investigators Consortium. 


\section{Poster Presentations}

\section{Acute Nicotine Toxicity Following Dermal Exposure to E- cigarette Liquid}

\section{Elizabeth Moore}

Banner - University Medical Center Phoenix, Phoenix, AZ, USA

Background: Electric cigarettes are gaining popularity, and liquid nicotine preparations or "vape juice" have been sold in formulations as high as $100 \mathrm{mg} / \mathrm{ml}$ of nicotine. There have been increased reports of liquid nicotine exposures reported to poison centers and multiple case reports of death associated with vape liquid ingestion. Time to onset of symptoms after oral exposure to nicotine is usually short. We present a case of delayed onset of nicotine toxicity in a pediatric patient following dermal exposure.

Hypothesis: Dermal exposure to liquid nicotine products without adequate decontamination can cause acute systemic toxicity.

Methods: This is a case report of a 15-month-old girl who developed delayed symptoms of nicotine toxicity after exposure to a $3 \%$ nicotine liquid solution. The patient was found playing with several bottles of "vape juice" and had the liquid on her hands, face, and chest. She was initially asymptomatic at home, but presented to an outside emergency department upon recommendation from the poison control center. Three hours after initial exposure and $2 \mathrm{~h}$ after her arrival to the ED, she developed multiple episodes of non-bilious, non-bloody vomiting, followed by excessive salivation, diaphoresis, and labile mood. Initial laboratory studies were negative. Toxicology was contacted and recommended transfer and decontamination. She was decontaminated with soap and water, and symptoms slowly improved. Upon her arrival to the tertiary care hospital $5 \mathrm{~h}$ after initial exposure, she was tachycardic (HR 145-186 bpm) and hypertensive $(128 / 75 \mathrm{~mm} / \mathrm{Hg})$. Vomiting, diaphoresis, and salivation had resolved. She did have a faint erythematous rash over exposed skin and labile mood. Her symptoms continued to improve and she was discharged home the day after initial exposure.

Discussion: Acute nicotine toxicity often presents with nausea, vomiting, and excessive salivation and can include lethargy, respiratory depression, seizures, and dysrhythmias. Previous case reports have described systemic toxicity after exposure to nicotine patches. This case is similar and suggests that there may be significant and potentially delayed onset of toxicity associated with dermal exposure to liquid nicotine products.

Conclusion: Dermal exposure to liquid nicotine products may produce significant systemic toxicity with delayed onset.

\section{A Toddler's Toxic Taste: Bradycardia after Brushing with Brimonidine}

Abby Montague, Stacey Bangh, Jon Cole

Minnesota Poison Control System, Minneapolis, MN, USA

Background: Brimonidine is a central alpha-2 adrenergic agonist. Mirvaso $®$ (Brimonidine) Topical Gel $0.33 \%$ is used for the treatment of facial erythema (rosacea) in adults. Toxicity from therapeutic doses of optic formulations of brimonidine has been reported; however, reports of exposures to dermal products are rare.

Hypothesis: Accidental ingestions of Mirvaso® (Brimonidine) Topical Gel $0.33 \%$ cause significant toxicity in children.

Methods: This is a single-patient chart review from a regional poison center and bedside toxicology consultation.

Results: A 4-year-old boy accidently used Mirvaso® topical gel $0.33 \%$ to brush his teeth. The drug had been given to the child's parent in clinic as a free sample. He spat out the "toothpaste" and his parent estimated only a taste was ingested. He was asymptomatic after ingestion with no oral pallor and went to school. However, $45 \mathrm{~min}$ after exposure, he started having somnolence and appeared pale. The school nurse measured a blood pressure at 70/40 $\mathrm{mmHg}$ and called EMS. They delivered bag mask ventilation during transport. On arrival to the emergency department, he was pale, limp, with GCS of 14, and capillary refill delay. Vitals were as follows: heart rate 69 beats/min, blood pressure $76 / 48 \mathrm{mmHg}$, respiratory rate 18 breaths/min, and oxygen saturation $99 \%$ on $4 \mathrm{~L} / \mathrm{min}$ nasal cannula. He was given $10 \mathrm{mg}$ of IV naloxone with no response. Atropine $0.3 \mathrm{mg}$ IV was administered with a rise to 149 beats $/ \mathrm{min}$ in heart rate and BP rise to $119 / 53$ but equivocal change in mental status. He was transferred to a pediatric intensive care unit and continued to have bradycardia and somnolence that slowly improved. He did not require repeat medication and toxicity resolved approximately $12 \mathrm{~h}$ after ingestion.

Discussion: Exploratory ingestions and therapeutic doses of optic brimonidine products have led to systemic toxicity in young children. The dermal preparation has the same toxic potential and should be considered dangerous in even taste-size doses in children. Free samples given in clinic should be accompanied by clear safety instructions.

Conclusion: Very small ingestions of brimonidine $0.33 \%$ topical formulation can cause significant toxicity in children.

\section{Characterization of Suspected Synthetic Cannabinoid Intoxications in Washington, DC}

Maryann Mazer-Amirshahi ${ }^{1,2}$, Bradley Gelfand ${ }^{2}$, Chikarlo Leak ${ }^{3}$, Samantha Tolliver $^{3}$, Diane Sauter ${ }^{1,2}$

${ }^{1}$ MedStar Washington Hospital Center, Washington, DC, USA

${ }^{2}$ Georgetown University School of Medicine, Washington, DC, USA

${ }^{3}$ Office of the Chief Medical Examiner, Washington, DC, USA

Background: There has been a surge of synthetic cannabinoid (SC) exposures in recent years, with more severe outcomes. At the same time, the constituents of such preparations are subject to variability and are rarely confirmed.

Study Objective: To characterize reported SC exposures presenting to emergency departments (EDs) in Washington, DC.

Methods: Patients who presented to two academic EDs with reported SC exposure from July 2015 to July 2016 had blood and/or urine samples obtained at the discretion of the provider during the course of routine care. De-identified samples were sent to the medical examiner's office for toxicology screening and laboratory confirmation with gas chromatography-mass spectrometry (GC/MS).

Results: A total of 132 cases were submitted for analysis. Four samples were pending or inadequate for analysis. Of the 128 samples with results, $72(56.3 \%)$ were positive for a SC on GC/MS, with $40(55.6 \%)$ positive for a SC alone, and $32(44.4 \%)$ for a SC and another substance. The most common SC detected was AB-FUBINACA $(28,38.9 \%)$, followed by ADB-FUBINACA and AB-CHMINACA 3-methyl-butanoic acid (15, $20.8 \%$ each), ADB-CHMINACA (14, 19.4\%), and 5-fluoro-PB-22 (8, $11.1 \%)$. Twenty-eight $(21.9 \%)$ cases were negative for SCs but positive for another substance. The most common non-SC substances detected on toxicology screening were tetrahydrocannabinol $(33,25.8 \%)$, phencyclidine $(23,18.0 \%)$, cocaine $(14,10.9 \%)$, opioids $(13,10.2 \%)$, and amphetamine derivatives $(6,4.7 \%)$. Thirty-six percent of samples were negative for both SCs and other drugs on the toxicology screen.

Discussion: Our study was limited because we could not examine the clinical effects of specific SCs. In addition, our findings could be subject to bias as more severe presentations would be more likely to have blood and/or urine specimens obtained by the treating physician. Our study took place in two urban academic EDs in Washington, DC, and our results may not be generalizable to other populations or different regions.

Conclusion: The individual SCs detected in our study were different than compounds detected in earlier studies (such as JWH compounds), suggesting that there has been a change in constituents, possibly to avoid regulation. Additional prospective research is needed to determine the clinical implications of these findings. 
16. Analysis of Look-Alike/Sound-Alike Medication Errors Involving Opioid Analgesics

Maryann Mazer-Amirshahi ${ }^{1}$, Katrina $\mathrm{Smith}^{2}$, Erica Savage ${ }^{2}$, Grace Tran $^{2}$, Allen Fong ${ }^{2}$, A. Zach Hettinger ${ }^{2}$

${ }^{1}$ MedStar Washington Hospital Center, Washington, DC, USA

${ }^{2}$ MedStar Health, Washinton, DC, USA

Background: Opioid analgesics are high-risk medication with the potential for adverse drug events. Look-Alike/Sound-Alike (LA/SA) medication errors account for $25 \%$ of medication errors. We reviewed patient safety events related to LA/SA medication errors that occurred involving opioid analgesics.

Study Hypothesis: Medication errors involving opioid analgesics would account for a significant portion of LA/SA errors and these errors would be associated with severe adverse effects.

Methods: Patient safety event (PSE) data was reviewed using text mining and natural language processing algorithms to search for LA/SA-related medication errors. The PSE database contains roughly 90,000 incidents from self-reported near misses and harm events by frontline staff in an academic healthcare system. Each case was reviewed independently by two subject matter experts and categorized by stage in the medication process (documentation, ordering, dispensing, or administration). Patient outcomes were examined.

Results: A total of 282 incidents met inclusion criteria for a LA/SA error, of which $57(20.2 \%)$ involved an opioid analgesic. The most common type of LA/SA errors involved immediate (IR)/extended release (ER) substitutions $(41,71.9 \%)$. Of these IR/ER errors, oxycodone products were implicated in $87.8 \%$ (36) of cases. Other IR/ER errors included morphine (4) and tapentadol (1). Additional LA/SA error pairs included hydromorphone/morphine (8.9\%), oxycodone with acetaminophen/ hydrocodone with acetaminophen (8.9\%), codeine with acetaminophen/ oxycodone with acetaminophen $(3.6 \%)$, oxycodone/oxycodone and acetaminophen (3.6\%), and other (5.3\%). Error frequency by stage was $5.3 \%$ documentation, $52.6 \%$ ordering, $21.1 \%$ dispensing, and $21.1 \%$ administration. Thirty $(52.6 \%)$ of LA/SA opioid errors reached the patient. Of errors that reached the patient, there were two adverse events: one change in mental status that did not require intervention and one severe respiratory depression that caused temporary harm requiring intubation.

Discussion: While limited by a small retrospective dataset, there were common themes suggesting interventions to prevent LA/SA errors involving opioid analgesics. Emphasis should be placed on improving ordering platforms as well as distinguishing between IR and ER formulations.

Conclusion: LA/SA errors involving opioid analgesics were more frequently associated with oxycodone products, particularly IR/ER formulations and most commonly occurred in the ordering stage of the medication process. Severe adverse effects were rare, but potentially life threatening.

\section{Varying Contribution of Ethanol to Serum Osmolality in Acute Intoxication}

Ryan Marino ${ }^{1}$, Todd Phillips ${ }^{2}$, Anthony Pizon ${ }^{2}$.

${ }^{T}$ Department of Emergency Medicine, University of Pittsburgh Medical Center, Pittsburgh, PA, USA

${ }^{2}$ Division of Medical Toxicology, Department of Emergency Medicine, University of Pittsburgh Medical Center, Pittsburgh, PA, USA

Background: Ethanol is the most common cause of osmolal gap elevations; however, current formulas do not accurately reflect ethanol's contribution to the osmolal gap. Unexplained elevation in osmolal gap in acute ethanol intoxication presents a clinical dilemma for practitioners.

Hypothesis: Patients with acute ethanol intoxication will have varying elevations in the osmolal gap, and current formulas do not accurately reflect ethanol's contribution to the osmolal gap.
Methods: We conducted a retrospective chart review of 11 hospitals from 2010 to 2015 . We included patients with the following laboratory testing performed concurrently: detectable serum ethanol level, serum osmolality, negative toxic alcohol panel and serum sodium, BUN, and glucose levels. Measured osmolality using freezing-point depression was compared with calculated osmolality using the standard formula $(2 \times[\mathrm{Na} \mathrm{mOsm} / \mathrm{L}]+[\mathrm{BUN} \mathrm{mg} / \mathrm{dL}] / 2.8+[$ glucose $\mathrm{mg} / \mathrm{dL}] / 18+$ $[\mathrm{ETOH} \mathrm{mg} / \mathrm{dL}] / 4.6)$. Osmolal gap $>10 \mathrm{mOsm} / \mathrm{kg}$ was considered abnormal. We used a linear regression to generate more precise correction factors for ethanol's contribution to osmolality at specific ethanol levels. Results: Of 82 patients identified, 53 had an elevated osmolal gap. In a subgroup of patients with high ethanol levels ( $>300 \mathrm{mg} / \mathrm{dL})$, the osmolal gap was elevated in 14 of 15 cases. We calculated a new divisor for ethanol's contribution to the osmolality formula, 3.57, which reduced the proportion of falsely elevated osmolal gaps from 64.6 to $20.7 \%$. Furthermore, we found that the divisor for ethanol's effect on osmolality had statistically significant variability at differing ethanol levels ( $p=0.049$ ). When ethanol's contribution to osmolality was stratified based on serum ethanol level and variable divisors applied at quantiles of $100 \mathrm{mg} / \mathrm{dL}$ ( 2.67 for $0-100,3.27$ for $100-200,3.53$ for $200-300,3.72$ for $>300$ ), the proportion of falsely elevated osmolal gaps was further reduced to $15.8 \%$.

Discussion: Calculated osmolal gap is an important screening tool for toxic alcohol ingestions. Costly therapies are often initiated based on elevations of osmolal gap alone, which is frequently unreliable. These data show that different divisors for ethanol's contribution to osmolality are required depending on ethanol level to more accurately reflect serum osmolality and reduce unnecessary interventions.

Conclusion: Ethanol has a variable effect on the osmolal gap at different levels and most evident at severely elevated levels.

\section{The Usefulness of Leukocytosis in Identifying Infections in the Overdose Patient}

Brian Marcks ${ }^{1}$, Anthony Pizon ${ }^{2}$, Brian Suffoletto

${ }^{1}$ South Shore Hospital, South Weymouth, MA, USA

${ }^{2}$ University of Pittsburgh Medical Center, Pittsburgh, PA, USA

Introduction: Elevated white blood cell (WBC) counts are frequently used by clinicians as an indicator of infections, despite WBC elevations potentially stemming from acute stress responses. Patients admitted for acute toxidromes are often treated for co-infections due to arbitrary WBC count elevations.

Hypothesis: We hypothesize WBC counts are less useful to differentiate infected from non-infected overdose patients when toxicities involve substances that trigger an acute stress response.

Methods: This is a retrospective review of consecutive acute overdose patients admitted to a medical toxicology service between April 2013 and August 2014. Exclusions included non-drug ingestions (e.g., methemoglobinemia, caustic ingestions), post-cardiac arrest patients, outpatients, withdrawal conditions, and patients lacking laboratory studies. Patients were grouped into 12 possible drug overdose categories (ethanol, stimulant, insulin, acetaminophen, opioid, antihistamine, NSAID, antihypertensive, benzodiazepine, antidepressant, antipsychotic, toxic alcohol). Primary outcome was mean WBC count on initial presentation based upon the presence or absence of infection. All statistical comparisons were made using a two-tailed Student's $t$ test.

Results: Of 1102 charts reviewed, 533 met inclusion/exclusion criteria. Mean age was 38 years (range 13-90). Patients were 274 females (51.4\%). The most common overdose was ethanol $(95 / 533 ; 17.8 \%)$, opioids $(60 / 533 ; 11.3 \%)$, and antidepressants $(59 / 533 ; 11.1 \%)$. Sixty-one $(11.4 \%)$ patients were diagnosed with infection during their hospital stay. Aspiration pneumonia $(48 / 61 ; 78.7 \%)$ and UTI $(7 / 61 ; 11.5 \%)$ were the most frequent infection types. For all patients, mean WBC count was higher in infection group (13.83 $( \pm 6.78 \mathrm{SD})$ vs $9.91 \mathrm{k} / \mathrm{mm}^{3}( \pm 5.95 \mathrm{SD})$; $p<0.001)$. 
In subgroup analysis, the WBC count was statistically higher when infection was present in only three subgroups: ethanol, opioid, and benzodiazepine (all $p<0.05)$.

Discussion: Generally, overdose patients presenting with an infection have higher WBC counts. However, in subgroup analysis, only the ethanol, opioid, and benzodiazepine groups have higher WBC counts with infections present. We believe this may be related to the lack of an acute stress response in these toxidromes unlike other toxic ingestions (i.e., stimulants). Conclusion: Overdose patients with infections tend to have higher WBC counts. However, future studies should examine how different toxic substances alter the immunologic system, which could have implications for infectious risk.

\section{A Case of Adult Lead Toxicity in Southeast Michigan: It's Not Just the Water in Flint}

Eric Malone, Luke Bisoski, Andrew King, Cynthia Aaron Wayne State University / Children's Hospital of Michigan Regional Poison Control Center, Detroit, MI, USA

Background: Lead is a neurotoxic heavy metal with multiple potential sources of exposure. While lead-contaminated drinking water in Southeast Michigan has been widely publicized, symptomatic lead toxicity has been rare. We present a patient with symptomatic elevated blood lead levels secondary to drinking from an imported mug.

Hypothesis: Chelation therapy is beneficial in adult patients with symptomatic lead toxicity.

Case Report: A 22-year-old woman presented for evaluation of intermittent abdominal pain. Diagnostic evaluation was notable for a $\mathrm{Hgb}$ of 8.5, normal electrolytes, AST 69, and ALT 106. Abdominal CT showed colitis. She was treated and discharged but later referred to a tertiary care center when her serum lead level returned at $106 \mathrm{mcg} / \mathrm{dL}$. Because of recent regional environmental lead contamination, this was thought to be secondary to contaminated ground water. At repeat presentation, she was clinically well with no encephalopathy or abdominal pain. Additional history suggested an imported Mexican mug in which she microwave heated her daily coffee as the likely source. Notable repeat laboratories were $\mathrm{Hgb} 7.5$, MCV 70.7, and serum lead level of $>100 \mathrm{mcg} / \mathrm{dL}$. Given her anemia, childbearing age, and intention for additional children, she was chelated with intravenous $\mathrm{CaNa}_{2}$ EDTA and oral succimer. She was discharged home to complete the 19-day oral succimer regimen. Diagnostic studies obtained during hospitalization suggested chronic exposure: total porphyrins $1400 \mathrm{mcg} / \mathrm{dL}(<80 \mathrm{mcg} / \mathrm{dL})$, zincprotoporphyrin $1256 \mathrm{mcg} / \mathrm{dL}(<60 \mathrm{mcg} / \mathrm{dL})$, free protoporphyrin $144 \mathrm{mcg} / \mathrm{dL}(<20 \mathrm{mcg} / \mathrm{dL})$, and free erythrocyte protoporphyrin $439 \mathrm{mcg} / \mathrm{dL}(0-35 \mathrm{mcg} / \mathrm{dL})$. Public health agency investigation conducted with X-ray fluorescence spectrometry confirmed the mug as a source of exposure. Household members, including the patient's children, were negative for elevated lead levels.

Discussion: We present a case of adult lead toxicity secondary to drinking coffee from an imported mug, who was successfully treated with chelation therapy.

Conclusions: An open differential should be maintained until there is confirmation of a definitive source of lead exposure. Inpatient intravenous chelation therapy should be considered even in non-encephalopathic patients of childbearing age with elevated lead levels and signs of systemic toxicity.

\section{An Assessment of the Diagnostic Utility of Pediatric Methanol and Ethylene Glycol Levels (Why is it Always a Gatorade $\AA$ bottle?)}

Eric Malone ${ }^{1}$, Luke Bisoski ${ }^{1}$, Michael Smith ${ }^{2}$, Cynthia Aaron ${ }^{1}$, Andrew King ${ }^{1}$

${ }^{1}$ Wayne State University / Children's Hospital of Michigan Regional Poison Control Center, Detroit, MI, USA

${ }^{2}$ William Beaumont Hospital, Royal Oak, MI, USA
Background: Methanol and ethylene glycol ingestions in pediatric patients are commonly accidental and unintentional. These ingestions are typically a "sip" or "swallow." Although potential toxicity of methanol and ethylene glycol is well-known, clinical thresholds for diagnostic testing and treatment can vary widely among medical toxicologists. There is little supporting data in this age group to provide an accurate assessment of pre-test probability.

Hypothesis: Innocent pediatric ingestions of methanol and ethylene glycol are usually benign, infrequently actionable, and rarely require antidotal treatment.

Methods: This was a retrospective chart review of all pediatric patients age 12 or less in whom serum methanol or ethylene glycol levels were obtained from a single reference laboratory during a 4-year time period. Serum levels were categorized as treatable or non-treatable based on serum level alone.

Results: Throughout the reviewed time period, there were 32 serum methanol concentrations obtained from 29 unique patient encounters. There were 37 serum ethylene glycol concentrations obtained from 31 unique patient encounters. Three (3/32) methanol concentrations were positive and the highest was $9.8 \mathrm{mg} / \mathrm{dL}$. All three positive values were from a single patient hospitalization. One (1/37) ethylene glycol concentration was positive at a level of $18.6 \mathrm{mg} / \mathrm{dL}$. In total, none of the serum methanol or ethylene glycol concentrations was potentially toxic based on previously described treatment thresholds.

Discussion: Innocent pediatric exposures to ethylene glycol and methanol are unlikely to be due to large ingestions with the intention of selfharm or intoxication. Potential treatment options can be highly resource intensive. Data to allow appropriate risk stratification of these patients is not currently available, but our experience suggests that these exposures are rarely actionable.

Conclusions: This single center, retrospective chart review is consistent with the clinical observation that the majority of pediatric ethylene glycol and methanol exposures do not require treatment. Additional multi-center studies are required to confirm these findings and to develop standardized treatment guidelines for such exposures.

\section{Drug-Induced Liver Injury: It's Not All Acetaminophen}

Eric Malone, Andrew King, Cynthia Aaron

Wayne State University / Children's Hospital of Michigan Regional Poison Control Center, Detroit, MI, USA

Background: Amoxicillin (an aminopenicilin) is a widely prescribed antibiotic with a side effect profile that is generally considered to be benign. We present a case of a child with amoxicillin-induced hepatitis and jaundice which worsened with in-hospital administration of piperacillin (a ureidopenicillin) and improved with discontinuation of antibiotics and initiation of steroid therapy.

Hypothesis: Amoxicillin-induced liver injury can be exacerbated by intravenous piperacillin.

Case Report: A 4-year-old previously healthy male with a past history significant only for atopic reactions to multiple xenobiotics was treated with amoxicillin for an upper respiratory tract infection. Following cessation of therapy, he became jaundiced. He was referred to the emergency department of a pediatric tertiary care hospital. He was found to have significant transaminase elevations with an AST of $1458 \mathrm{U} / \mathrm{L}$ and ALT of $1373 \mathrm{U} / \mathrm{L}$. Total bilirubin and direct bilirubin were elevated at 4.2 and $2.4 \mathrm{mg} / \mathrm{dL}$, respectively. After transabdominal ultrasound was suspicious for acalculous cholecystitis, he was treated with intravenous piperacillin/ tazobactam. Serum transaminases rapidly increased to AST $>3500$ and to ALT $>2000$. Total and direct bilirubin peaked at 14.7 and $12.1 \mathrm{mg} / \mathrm{dL}$. INR was 1.3, PTT was $33.0 \mathrm{~s}$, and PT was $15.3 \mathrm{~s}$. Extensive evaluation, including MRCP, viral titers, and serum markers, for autoimmune diseases was non-diagnostic. Liver biopsy was suggestive of autoimmune or toxic causes. The patient's physical examination, aside from marked jaundice, was unremarkable. Penicillin-induced cholestatic hepatitis was 
suspected, piperacillin/tazobactam was discontinued, and intravenous steroids were started. All laboratory parameters rapidly improved and the patient was discharged home.

Discussion: Amoxicillin monotherapy is rarely implicated in liver injury with evidence of both hepatocellular damage and cholestasis. In this case, amoxicillin-induced liver injury and cholestasis worsened following an unintentional challenge with piperacillin, suggesting potential cross-reactivity. Conclusion: Amoxicillin monotherapy can result in significant transaminase elevations and hyperbilirubinemia. Idiopathic drug reactions should be considered in patients presenting with hepatitis, jaundice, and no other identifiable etiology. Other classes of penicillin antibiotics should be specifically avoided in patients with a history of amoxicillin-induced liver injury.

\section{Severe Thallium Toxicity Treated Successfully with Prussian Blue and CVVH}

Eric Malone $^{1}$, Luke Bisoski ${ }^{1}$, Lydia Baltarowich ${ }^{2}$, Cynthia Aaron ${ }^{1}$

${ }^{T}$ Wayne State University / Children's Hospital of Michigan Regional

Poison Control Center, Detroit, MI, USA

${ }^{2}$ Henry Ford Health System, Detroit, MI, USA

Background: Thallium is a transition metal implicated in intentional and unintentional toxicity secondary to historical medical uses, industrial uses, intentional poisoning, and accidental exposure. We present a patient with severe thallium toxicity who was treated successfully with multiple modalities including Prussian blue and continuous veno-venous hemofiltration $(\mathrm{CVVH})$ with eventual clinical improvement.

Case Report: A previously healthy 41-year-old man presented with severe, symmetric, progressive bilateral lower extremity sensorimotor neuropathy. He was diagnosed with Guillain-Barre syndrome, treated with intravenous immunoglobulin and steroids, and transferred to a rehabilitation facility. Subsequently, he developed progressively worsening encephalopathy and was readmitted. Comprehensive evaluation was nondiagnostic. After alopecia developed, thallium toxicity was suspected. A serum heavy metal test was obtained and was notable for a thallium level of $158 \mathrm{ng} / \mathrm{mL}(0-1 \mathrm{ng} / \mathrm{mL})$.

The patient was started on CVVH and received multi-dose activated charcoal until Prussian blue was obtained from the CDC. Both Prussian blue and CVVH were continued for 3 weeks. His inpatient hospital course was complicated by waxing and waning encephalopathy and severe bilateral lower extremity pain. Repeat thallium levels initially increased to $197 \mathrm{ng} / \mathrm{mL} 4$ days later following initiation of therapy. Serum thallium levels then trended down to $53 \mathrm{ng} / \mathrm{mL}$ after 1 week and $6 \mathrm{ng} / \mathrm{mL}$ after 3 weeks. Urine thallium levels declined from $>800 \mathrm{mcg} / \mathrm{L}$ $(0-1 \mathrm{mcg} / \mathrm{L})$ to $15 \mathrm{mcg} / \mathrm{L}$ over 3 weeks. The patient had significant improvement in neurologic symptoms and encephalopathy and was discharged to a rehabilitation center.

Discussion: Thallium toxicity should be suspected in patients with encephalopathy, painful neuropathy, and alopecia. Treatment consists of oral Prussian blue, which binds recirculating thallium in the GI tract, and CVVH, which enhances the elimination of thallium.

Conclusions: This case illustrates that aggressive treatment, even weeks after initial presentation, can decrease the thallium body burden and result in clinical improvement of neurologic toxicity.

\section{West African Bush Viper (Atheris chlorechis) Coagulopathy Treated Successfully with Polyvalent Antivenom}

Eric Malone $^{1}$, Bram Dolcourt ${ }^{1}$, Cynthia Aaron ${ }^{1}$, Bryan Judge ${ }^{2}$

${ }^{T}$ Wayne State University / Children's Hospital of Michigan Regional Poison Control Center, Detroit MI, USA

${ }^{2}$ Grand Rapids Medical Education Partners / Michigan State University Emergency Medicine Residency Program, Grand Rapids, MI, USA

Background: Atheris chlorechis is an arboreal species of bush viper endemic to portions of West Africa. The genus Atheris is phylogenetically diverse due to unique regional biogeographic characteristics. Cases of envenomation and successful treatment of coagulopathy have been inadequately described. We present a case of envenomation by A. chlorechis with a successful antivenom therapy.

Hypothesis: Polyvalent Echis antivenom is effective for the treatment of coagulopathy following envenomation by $A$. chlorechis.

Case Report: A previously healthy 32-year-old male amateur snake keeper experienced two bites in rapid succession from a pet West African bush viper to the right hand dorsal web space between the first and second digits. At presentation, there was pain and swelling at the envenomation sites. Laboratory values included INR of $1.0, \mathrm{Hgb}$ of 13.4 , and platelet count of 127,000 . Over $8 \mathrm{~h}$, the patient developed coagulopathy and progressive swelling of his right upper extremity. Platelet count and Hgb remained stable, but INR increased to 6.7 with fibrinogen of $341 \mathrm{mg} / \mathrm{dL}$. Antivenom was not immediately available, so he was treated with fresh frozen plasma. His coagulopathy continued to worsen with INR increasing to $>12$ and PTT $>120$ at approximately $18 \mathrm{~h}$ post-envenomation. He remained hemodynamically stable but experienced bleeding from his gums and puncture wounds.

Following treatment with polyvalent Echis antivenom, obtained from an out-of-state reptile house, there was improvement in the patient's coagulopathy. INR decreased to 2.4 and PTT decreased to 28 . Fibrin monomers remained elevated and platelet count decreased to 66,000 . Creatinine increased from a baseline of 0.8 to $3.1 \mathrm{mg} / \mathrm{dL}$. Following symptomatic improvement, the patient left against medical advice on hospital day 2 .

Discussion: Envemonations by A. chlorechis are poorly described. We present a case of coagulopathy treated successfully with polyvalent Echis antivenom.

Conclusion: Polyvalent Echis antivenom may be effective in ameliorating coagulopathy from envenomation by $A$. chlorechis.

\section{Neurotoxicity and Wound Infection Following Monocled Cobra} Envenomation in the US

Hannah Malashock $^{1,2}$, Anne-Michelle Ruha ${ }^{1,2}$, Kimberlie Graeme ${ }^{1,2}$, Eleanor Oakley ${ }^{1,2}$, Elizabeth Moore ${ }^{1,2}$, David Watts ${ }^{1,2}$

${ }^{1}$ Department of Medical Toxicology, Banner - University Medical Center Phoenix, Phoenix, AZ, USA

${ }^{2}$ Center for Toxicology \& Pharmacology Education and Research, University of Arizona College of Medicine - Phoenix, Phoenix, AZ, USA

Background: The monocled cobra (Naja kaouthia), native to South and Southeast Asia, is known to cause neurotoxicity and tissue necrosis. Standard treatment is administration of specific antivenom; however, such antivenom is not widely available in the US. We report clinical effects and response to treatment following a monocled cobra envenomation that occurred in the US where specific antivenom was not readily available.

Case Report: A 72-year-old man was bitten by a captive monocled cobra. He immediately developed nausea and vomiting and was transported to an ED by EMS. On arrival, he reported wound pain, abdominal pain, and fatigue, and shortly thereafter developed ptosis. The bite site revealed two $1-\mathrm{cm}$ dark lesions with surrounding erythema. Initial vital signs were unremarkable; however, over the next few hours, he became progressively more hypertensive and tachycardic with a blood pressure reaching 183/105 and heart rate of $117 \mathrm{bpm}$. Neurotoxicity progressed to bulbar paralysis and respiratory depression, and was unresponsive to four vials SAIMR Polyvalent antivenom and neostigmine $0.5 \mathrm{mg}$ IV. He was intubated, and descending paralysis continued despite a trial of two vials MENA Inoserp AV. Eight hours after the bite, speciesspecific Thai cobra AV was obtained via private air transport from an outof-state zoo. Within several hours of this antivenom, his neurotoxicity completely resolved and he was extubated. Wound erythema and swelling progressed to a size of $13 \times 23 \mathrm{~cm}$, and the initial darkened area became necrotic. Oral trimethoprim-sulfamethoxazole was started empirically about $40 \mathrm{~h}$ after the bite but at $48 \mathrm{~h}$, he developed a temperature of 
$38.6^{\circ} \mathrm{C}$ and IV antibiotics vancomycin, gentamicin, and piperacillintazobactam were started. Wound cultures grew Morganella morganii. Fever resolved, erythema improved, and he was discharged home with oral ciprofloxacin based on sensitivities.

Conclusion: Monocled cobra envenomations can cause severe neurotoxicity and wound infections associated with particular organisms, including M. morganii. While native snake envenomations are rarely associated with infection, cobra bites should be monitored closely for infection. Despite challenges in locating and transporting exotic antivenoms emergently within the US, it is important to obtain species-specific antivenom to effectively treat toxicity.

\section{A Comparison of Drug-Related and Other Cause Compartment Syndrome}

Alicia Lydecker ${ }^{1}$, Vincent $\mathrm{Kan}^{1}$, Louise Maranda ${ }^{1}$, Kavita Babu ${ }^{2}$, Jonathan Slutzman ${ }^{2}$

${ }^{1}$ University of Massachusetts Medical School, Worcester, MA, USA

${ }^{2}$ University of Massachusetts Medical Center, Worcester, MA, USA

Background: Traditionally seen after traumatic injury, compartment syndrome (CS) is increasingly being reported as a sequela of drug overdose. In this study, we compare drug-related CS to non-drug-related CS.

Hypothesis/Research Question: How do patients with drug-related CS differ in comparison to those without drug overdose?

Methods: This retrospective chart review included adult subjects who received a diagnosis of acute CS at a tertiary care medical center. Patients with orbital or abdominal CS were excluded. Drug exposure was identified by history or quantitative laboratory evidence of recent ethanol or drug use. Patients were further stratified into traumatic and atraumatic arms. Data were abstracted for demographics, etiology, laboratory values, patient outcomes, admission and discharge diagnoses, hospital length/cost of stay, and discharge medications. Results were analyzed using comparative statistics. This study received Institutional Review Board approval.

Results: Between 1/1/12 and 10/10/15, 117 cases met inclusion criteria. In patients with traumatic CS (75/117), there were no significant differences between the drug-related and non-drug-related arms. In patients with nontraumatic CS (42/117), 15 were drug-associated, including opioids $(60 \%)$, benzodiazepines (33\%), ethanol (33\%), antidepressants (20\%), antipsychotics (13\%), gabapentin (13\%), cocaine (13\%), and other $(27 \%)$. Patients with drug-associated nontraumatic CS were younger (41.1 vs 58.3 years, $p=.001)$; had higher peak CPKs $(52,733$ vs $19,775 \mathrm{U} / \mathrm{L}, p=.006$ ); higher frequency of rhabdomyolysis (100 vs $37 \%, p<0.001$ ); more acute renal failure ( 80 vs $44 \%, p=0.027$ ); and a higher incidence of hyperkalemia ( $67 \mathrm{vs} 26 \%, p=0.012$ ) than non-drugexposed counterparts. There were nonsignificant trends towards longer lengths of stay (20.2 vs 11.7 days) and higher costs of hospitalization $(\$ 51,651$ vs $\$ 42,906)$. Of the $111 / 117$ patients who survived, 106 were discharged with opioids

Discussion: Drug intoxication had no significant associations in patients with traumatic CS, perhaps because the intoxication was coincidental or a risk factor for injury. Patients with nontraumatic drug-related CS tended to have periods of "down" time, with more muscle necrosis and associated complications.

Conclusion: Drug intoxication or overdose is associated with higher morbidity in patients with nontraumatic CS.

\section{Assessment of Emergency Medicine Providers' Knowledge, Utilization and Prescribing of Naloxone in the Emergency Department}

Jennifer Love, Rachel Graves, David Jang, Frances Shofer, Jeanmarie Perrone

Perelman School of Medicine at the University of Pennsylvania, Department of Emergency Medicine, Philadelphia, PA, USA
Background: With the growing volume of opioid-related poisonings treated in the emergency department (ED), it is valuable to understand ED providers' knowledge and experience with naloxone prior to implementing a naloxone dispensing program. Previous studies have examined primary physicians' opinions on naloxone distribution and ED-based naloxone administration. However, few studies have examined ED providers' experience prescribing naloxone from the ED for outpatient use.

Research Questions: Do ED physicians prescribe naloxone from the ED? Are ED providers knowledgeable about extra-hospital naloxone? What knowledge gaps can be targeted to enhance additional harmreduction strategies for these patients?

Methods: A 4-point Likert scale 16-question survey was iteratively developed and piloted; it was subsequently distributed via email and in-person to a convenience sample of ED providers in a single, academic, urban ED in fall 2016. ED providers included attending physicians, ED residents, and nurses. Standard summary statistics and chi-square test were performed.

Results: Survey response rate was 57\% (86/150). Respondents were 50\% male, $31 \%$ attendings, $22 \%$ ED residents, and $47 \%$ nurses. Ninety-nine of providers had administered naloxone in the ED. Of 46 physicians, 43 were willing to prescribe naloxone to patients or family members at discharge, but only 3 physicians had previously prescribed naloxone. ED providers never or rarely asked patients about access to clean needles $(62 \%)$ or witnessing drug overdoses $(74 \%)$. Conversely, providers frequently asked about origins of their addiction $(62 \%)$ or past rehabilitation admissions (85\%). ED nurses were more likely than the physicians to always query patients about addiction history ( $31 \mathrm{vs} 4 \%, p=0.008$ ) and past drug overdoses (60 vs 39\%, $p=0.03$ ).

Discussion: ED providers have experience using naloxone in the ED but are more limited in naloxone prescribing at discharge and bedside harmreduction education. Provider knowledge gaps identified can augment harm reduction efforts by providing enhanced naloxone prescribing with resource information and by encouraging ED bedside provider-patient dialogue about risk behavior.

Conclusion: In the process of implementing an ED-based naloxone dispensing program, our survey of ED providers highlights opportunities to improve dialogue, resources, and naloxone information for patients with opioid addiction.

\section{Assessing Bleeding Risk In Pediatric Patients With Accidental Ingestion Of Novel Anticoagulant And Antiplatelet Medications}

Michael Levine $^{1}$, Anthony Pizon ${ }^{2}$, Michael Beuhler $^{3}$, Lee Cantrel ${ }^{4}$, Aaron Skolnik ${ }^{2}$, Meghan Spyres ${ }^{1}$, Fiona Garlich ${ }^{1}$, Dan Brooks ${ }^{5}$

${ }^{1}$ University of Southern California, Los Angeles, CA, USA

${ }^{2}$ University of Pittsburgh, Pittsburgh, PA, USA

${ }^{3}$ Carolinas Medical Center, Charlotte, NC, USA

${ }^{4}$ California Poison Control System, San Diego, CA, USA

${ }^{5}$ Banner University Medical Center Phoenix, Phoenix, AZ, USA

Background: In recent years, there has been an increase in the use of antiplatelet agents and novel anticoagulants. The natural exploratory nature of toddlers makes young children particularly at risk for accidental ingestions. There is little information on bleeding events and outcomes of children following exploratory ingestions of these agents.

Methods: This retrospective review of children age $<6$ years involves calls to seven poison control centers (PCC) in four states. A computerized search identified all calls from 2005 to 2014 involving the following drugs: apixaban, clopidogrel, dabigatran, edoxaban, prasugrel, rivaroxaban, and ticagrelor. Adult patients were excluded, as were all intentional ingestions. Thus, this study focused exclusively on accidental pediatric ingestions. Follow-up was defined a priori as at least one phone call occurring at least $4 \mathrm{~h}$ after the initial call.

Results: During the study, a total of 627 calls were identified. The median (interquartile range) age was $2(1.5-2)$ years. Males accounted for 383 $(61 \%)$ of calls. Follow-up was available for $284(46 \%)$ of calls. Among 
these 284 patients, the duration of follow-up was $<24 \mathrm{~h}$ in all (98.4\%) except 5 cases. The rates of bleeding and the corresponding number of cases are as follows: apixaban (0/7); clopidogrel (0/553); dabigatran $(0 / 19)$; prasugrel (0/17); rivaroxaban ( $0 / 27)$; and ticagrelor $(0 / 4)$. No cases of edoxaban were identified.

Limitations: The primary limitation is this study relied on retrospective PCC data with no confirmation of exposure and no direct patient evaluations. Furthermore, many patients had no follow-up and of those who did, the majority had less than $24 \mathrm{~h}$ of follow-up.

Conclusion: In this study of novel anticoagulants and antiplatelet agents, no cases of bleeding were identified among the 627 accidental pediatric ingestions.

\section{Assessing The Involvement Of A Medical Toxicologist In The Care Of Rattlesnake Envenomated Patients}

Michael Levine $^{1}$, Steve Offerman ${ }^{2}$, Rais Vohra $^{3}$, Brian Wolk ${ }^{4}$, Jeff Lapoint $^{3}$, Dan Quan ${ }^{6}$, Meghan Spyres ${ }^{1}$, Fiona Garlich ${ }^{1}$, Frank LoVecchio $^{7}$

${ }^{1}$ University of Southern California, Los Angeles, CA, USA

${ }^{2}$ Kaiser Northern California, Sacramento, CA, USA

${ }^{3}$ UCSF Fresno Medical Education Program, Fresno, CA, USA

${ }^{4}$ Loma Linda University School of Medicine, Loma Linda, CA, USA

${ }^{5}$ Southern California Permanente Medical Group, San Diego, CA, USA

${ }^{6}$ Maricopa Integrated Health System, Phoenix, AZ, USA

${ }^{7}$ Banner-University Medical Center Phoenix, Phoenix, AZ, USA

Background: Rattlesnake envenomations are common. The impact of a medical toxicologist in the care of a patient with a rattlesnake envenomation is unknown. The primary purpose of this study was to evaluate the impact of a toxicology service in the care of these patients.

Methods: This study compares the care of patients presenting with rattlesnake envenomation before and after a toxicology service was created at six different medical centers. Data were collected for the 2 years before and 2 years after the toxicology service was created. Each center started the toxicology service at different times (ranging from 2008 through 2013).

Results: A total of 390 subjects were identified (210 pre-toxicologist, 180 post-toxicologist). The median (IQR) age was 46 (24-47) years; $75.6 \%$ were male and $60.7 \%$ had upper extremity bites. There were no differences in demographics, bite characteristics, transfusion of blood products, or surgical intervention between the two groups. The mean number of vials of CroFab in the pre-group was 11.1 (95\% CI 10.1-12.1) compared to 13.6 (95\% CI $12.43-14.77$ ) in the post-group. However, the mean length of stay decreased significantly from 72.2 (95\% CI 65.7-78.6) h in the pre-group to 47.6 (95\% CI 44.1-51.3) $\mathrm{h}$ in the post-group. Readmission occurred in $24(11.4 \%)$ of the pre-group, compared with 26 $(14.4 \%)$ of the post-group $(p=0.44)$.

Conclusion: The establishment of a toxicology service was associated with slightly increased utilization of $\mathrm{CroFab}$ but at a significantly reduced length of stay. This decreased length of stay was not associated with an increased rate of readmission.

\section{Comparison Of Pediatric Vs. Adult Rattlesnake Envenomations: The ToxIC Experience}

Michael Levine ${ }^{1}$, Michelle Ruha ${ }^{2}$, Rais Vohra ${ }^{3}$, Brian Wolk ${ }^{4}$, Meghan Spyres $^{1}$, Jeffery Brent ${ }^{5}$, Paul Wax ${ }^{6}$, Sharan Campleman ${ }^{7}$, On Behalf of the Toxicology Investigators Consortium (ToxIC) NASBR

${ }^{1}$ University of Southern California, Los Angeles, CA, USA

${ }^{2}$ Banner-University Medical Center Phoenix, Phoenix, AZ, USA

${ }^{3}$ UCSF Fresno Medical Education Program, Fresno, CA, USA

${ }^{4}$ Loma Linda University School of Medicine, Loma Linda, CA, USA

${ }^{5}$ University of Colorado, Denver, CO, USA

${ }^{6}$ University of Texas Southwestern Medical Center, Dallas, TX, USA

${ }^{7}$ American College of Medical Toxicology, Phoenix, AZ, USA
Background: Little data objectively compares pediatric and adult patients after rattlesnake envenomation. The objective of this study is to utilize the North American Snake Bite Registry (NASBR), to compare envenomation severity and treatment characteristics between adult and pediatric patients.

Methods: The NASBR is a prospectively collected registry of patients evaluated by medical toxicologists following snakebite. This study compared pediatric (age $<18$ years) vs. adult patients who presented with a known or suspected rattlesnake bite between 1/1/2013 and 12/31/2015. Severity was compared between adult and pediatric patients with regard to the following parameters: hematologic toxicity (defined a priori as platelets $<120,000 / \mathrm{uL}$, fibrinogen $<170 \mathrm{mg} / \mathrm{dL}$, or PT $>15 \mathrm{~s}$ ), length of stay (in days), anaphylactoid reactions (defined a priori as hypotension and/or angioedema), and the total number of vials of antivenom administered. Gastrointestinal symptoms were defined as the presence of nausea/vomiting prior to opiates, or diarrhea. Independent associations were assessed via the chi-squared and Fisher exact test (as appropriate) for categorical variables. Medians with interquartile ranges were used to assess normally distributed ordinal data.

Results: A total of 256 (63 pediatric; 193 adult) patients were identified from 12 centers throughout the United States. Males accounted for $65 \%$ of pediatric and $78 \%$ of adult bites $(p=0.001)$. Upper extremity bites were more common in adult patients compared with pediatric patients (58 vs. $24 \% ; p=0.0001)$. There was no statistical difference in the prevalence of gastrointestinal symptoms (6.2 vs. 14.3\%), anaphylactoid reactions (6.7 vs. $4.8 \%)$, bleeding events (9.3 vs. $4.7 \%)$, or hematologic toxicity (44.6 vs. $52.4 \%$ ) between adult and pediatric patients, respectively. Comparing adult to pediatric patients, there was no difference in the median [IQR] length of stay (2 [1-2] vs. 2 [1-2]) or the mean total vials of antivenom during the index hospitalization (11.9 vs. 11.5), respectively. In this dataset, follow-up data was available for $73 \%$ of all cases with $27.7 \%$ indicating at least one instance of late hematologic toxicity (20.9 and $29.7 \%$ ( $p=$ NS) among pediatric and adult cases, respectively).

Conclusion: Pediatric patients are more likely to have lower extremity bites than adult patients. Toxicity and treatment are similar among pediatric and adult patients.

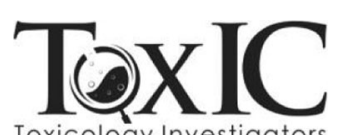

This research was performed in collaboration with the ACMT Toxicology Investigators Consortium.

Toxicology Investigators

\section{Acceptance Among Heroin Users of Advanced Technology in Studying Naloxone Distribution Programs}

Jeffrey Lai ${ }^{1}$, Peter Chai ${ }^{1}$, Katherine Boyle ${ }^{2,3}$, Edward Boyer ${ }^{1}$

${ }^{T}$ University of Massachusetts Medical School, Worcester, MA, USA

${ }^{2}$ Beth Israel Deaconess Medical Center, Boston, MA, USA

${ }^{3}$ Harvard Medical School, Boston, MA, USA

Objective: Heroin overdose deaths continue to rise, prompting implementation of "bystander" naloxone programs in community and emergency department (ED) settings. Rigorous data demonstrating the outcomes of naloxone rescue kits dispensed to heroin users are lacking. Advanced technology-based monitoring may help monitor efficacy of these programs. We sought to determine heroin users' acceptance of bystander naloxone kits and attitudes toward use of advanced technology to study the kits' community penetrance and geographic distribution patterns.

Methods: A convenience sample of 23 adults in the ED with a complaint related to heroin use completed a survey regarding naloxone distribution programs and familiarity with advanced technology such as low-energy Bluetooth (BLE) tracking beacons. Participants were asked about duration of heroin use, other substances used (excluding alcohol/tobacco), 
familiarity with naloxone, and acceptability of dispensing BLE-tagged "smart naloxone kits" to characterize effectiveness of naloxone distribution programs.

Results: Participants were $52 \%$ male, median age 32 years, median heroin use duration 4 years. Seventy-eight percent used other substances, including cocaine (61\%), benzodiazepines (30\%), and marijuana (22\%). Eightyseven percent had previously overdosed on heroin. Eighty-three percent knew someone who needed naloxone. Sixty-five percent had needed to administer naloxone personally. Sixty-five percent had used advanced technology (e.g., GPS, Bluetooth, smartphones) within the past month. Naloxone distribution programs were favorably received: $52 \%$ perfectly acceptable, $26 \%$ acceptable, and $21 \%$ slightly acceptable. BLE technology was widely accepted: $22 \%$ felt they were more likely to carry BLEtagged kits, while $70 \%$ felt BLE would not affect whether they carry the kits and $9 \%$ reported that BLE would deter them from using the kits.

Conclusion: Our data demonstrate that heroin users are accepting advanced technology deployment to study the efficacy of naloxone distribution programs. The readiness of individuals with substance use to accept BLE technology is important for the development of further iterations of advanced monitoring devices to detect and respond to opioid overdose.

\section{Survey of Emergency Department Physicians on Poison Center Consultation}

Ron Kirschner ${ }^{1,2}$, Russell Buzalko ${ }^{1}$, Karen Smith ${ }^{2}$

${ }^{T}$ University of Nebraska Medical Center, Omaha, NE, USA

${ }^{2}$ Nebraska Regional Poison Center, Omaha, NE, USA

Background: Poison center (PC) consultation can improve outcomes for patients with overdose or poisoning; however, emergency department physicians (EDPs) may be hesitant to contact the PC due to the perceived time requirement.

Research Question: How do EDPs view the efficiency of the PC telephone consultation process and what modifications would they recommend to improve the ED-PC interaction?

Methods: An electronic survey link was sent to the regional American College of Emergency Physicians chapter and distributed to members $(n=283)$ throughout one PC's service area. Providers were queried about their perceptions of the $\mathrm{PC}$ telephone consultation process and their suggestions for improvement.

Results: Fifty-eight EDPs completed the survey (20.5\% response rate). When asked whether they viewed the ED-PC consultation process as efficient, $86.2 \%$ responded "always" or "usually" efficient. Telephone hold times were perceived as always $(52.4 \%)$ or usually $(47.6 \%)$ reasonable. $74.1 \%$ of respondents thought the number of questions asked by the PC was "just right" while $25.9 \%$ felt it was "too many" or "far too many." Most (61.8\%) responded that the anticipated length of time to discuss a case was never a barrier to contacting the PC; however, $29.1 \%$ indicated it was sometimes or occasionally a barrier, and $9.1 \%$ indicated it was usually or always a barrier. When asked about alternative modes of communication for discussing cases, $96.3 \%$ of respondents preferred the telephone over fax, e-mail, or texting. However, for simply reporting a case when no recommendations were sought, communication preferences were more evenly distributed.

Discussion: The majority of respondents conveyed satisfaction with the PC telephone consultation process; however, some indicated that the time required to discuss a case with the PC was too long and sometimes a barrier to them calling. In addition, EDPs expressed interest in a variety of options to communicate with the PC about cases. This survey was sent to EDPs served by a single poison center; therefore, results may not be applicable nationwide.

Conclusion: Streamlining the ED-PC consultation process and exploring alternative communication methods such as e-mail or texting for less complex cases may reduce barriers to EDPs contacting the poison center.

\section{Bradycardia and Hypotension after $20 \mathrm{mg}$ of IV Olanzapine}

A. Mariah Kirsch ${ }^{1}$, Lisa Carlson ${ }^{1}$, David Plummer ${ }^{1}$, Jon Cole ${ }^{1,2}$

${ }^{T}$ Hennepin County Medical Center, Minneapolis, MN, USA

${ }^{2}$ Minnesota Poison Control System, Minneapolis, MN, USA

Background: Olanzapine is a second-generation antipsychotic available as an immediate-release formulation that is FDA approved for intramuscular use only. This same formulation of olanzapine, however, is increasingly being used intravenously (IV). The maximum studied IV dose in published literature is $10 \mathrm{mg}$; scant data are available on larger IV doses. Therapeutic doses of olanzapine have minimal cardiovascular effects. Postural hypotension from $\alpha_{1}$-adrenergic blockade with reflex tachycardia is well described; however, simultaneous bradycardia and hypotension are also reported.

Hypothesis: Large doses of IV olanzapine may result in bradycardia and hypotension.

Methods: This is a single patient chart review. A 25-year-old man presented to the emergency department acutely agitated after sustaining a stab wound to his abdomen. Initial vital signs were as follows: pulse 114 beats/min, blood pressure $154 / 83 \mathrm{mmHg}$, and pulse oximetry $100 \%$. His hospital stay was not complicated by hemorrhagic shock. His agitation was treated with $20 \mathrm{mg}$ of IV olanzapine given in two 10mg doses $8 \mathrm{~min}$ apart. He also received $10 \mathrm{mg}$ of IV haloperidol $18 \mathrm{~min}$ after the first olanzapine dose.

Results: The patient became sedate, hypotensive, and bradycardic approximately $20 \mathrm{~min}$ after the first olanzapine dose. Vital signs at that time were as follows: pulse 47 beats $/ \mathrm{min}$, blood pressure $86 / 38 \mathrm{mmHg}$, and pulse oximetry $92 \%$. An ECG confirmed sinus bradycardia. Labs revealed a serum potassium of $2.8 \mathrm{mEq} / \mathrm{L}$ and a blood ethanol concentration of $128 \mathrm{mg} / \mathrm{dL}$. Interventions included electrolyte replacement, $2 \mathrm{~L}$ of IV crystalloid, and supplemental oxygen. Vital signs normalized without additional interventions $1 \mathrm{~h}$ later.

Discussion: Reports of bradycardia accompanied by hypotension after administration of olanzapine have been reported after both therapeutic dosing and overdose, though rarely. No clear causative mechanism is known for this phenomenon, though sympatholysis has been posited. As IV use of olanzapine becomes more common, clinicians should be aware of and monitor for bradycardia as escalating doses are administered. As with previously reported cases of bradycardia associated with olanzapine, this patient responded well to supportive care.

Conclusion: Clinicians should monitor for bradycardia and hypotension after the administration of large doses of IV olanzapine.

\section{Stoned by the Philosopher: The Tragic Case of the Mercurious Neighbor}

Andrew King, Cynthia Aaron

Wayne State University / Children's Hospital of Michigan Regional Poison Control Center, Detroit, MI, USA

Background: In alchemy, the process of chrysopoeia is the act of transmuting a "base" metal (e.g., lead or mercury) into a "noble" metal (e.g., gold or silver). We report one attempt at mercury transmutation by an amateur alchemist that resulted in death and EPA-directed clean up.

Hypothesis: Attempted creation of gold from mercury can be lethal and results in severe collateral damage.

Methods: This is a single case report of a man who attempted to create gold by boiling mercury with calcium hydroxide. His parents found him dead in his condominium and tried to clean up the scene themselves. His official autopsy report deemed his cause of death as mercury toxicity and the manner of death accidental.

Results: $2 / 26$ units in his building were condemned; three additional units had elevated mercury levels. The EPA-directed remediation found extremely elevated ambient mercury concentrations throughout the decedent's residence, personal items, clothes, and car. Crews deconstructed the condominium unit down to the studs and base floors for adequate decontamination. 
Significantly elevated mercury concentrations were found in the adjacent unit that was also condemned and deconstructed to the studs. Officials theorized that the lack of a firewall and leakage between the shared heating pipes and holes around electrical outlets contributed to mercury diffusion. Fortunately, that dwelling was unoccupied. A sink two apartments away used during initial clean up required removal as hazardous waste. The dumpster used to throw away the decedent's belongings was contaminated and was sealed and transported as hazardous materials. His father's washing machine also required hazardous disposal.

Discussion: Elemental mercury inhalation can lead to severe toxicity and death. The potential for collateral contamination may be underappreciated especially since mercury can track into unaffected areas through contaminated clothing and shoes. This should be considered when caring for poisoned patients. Prompt involvement of public health officials is essential to evaluate the scene and encourage others to seek medical evaluation. Conclusion: The creation of elemental mercury in a condominium or apartment can permeate through walls potentially causing toxicity in adjoining residences. Attempts at cleaning by laypersons do more harm than good.

\section{Does Targeted Temperature Management Improve Hospital Survival For Drug-Overdose Related Cardiac Arrest?}

Sharaf Khan ${ }^{1}$, Chad Meyers ${ }^{2}$, Suzanne Bentley ${ }^{2}$, Yanoh Jalloh ${ }^{1}$, Alex Manini ${ }^{2}$

${ }^{T}$ The Icahn School of Medicine at Mount Sinai, New York, NY, USA

${ }^{2}$ The Icahn School of Medicine at Mount Sinai, Elmhurst Hospital Center, New York, NY, USA

Background: Drug overdose is a leading cause of out-of-hospital cardiac arrest $(\mathrm{OHCA})$ among young adults. There is limited data regarding the efficacy of targeted temperature management (TTM) in this population. Research Question: In emergency department (ED) patients, does TTM improve hospital survival from overdose-related cardiac arrest?

Methods: We performed a retrospective chart review of consecutive cardiac arrests presenting to an urban tertiary care hospital ED from 2011 to 2015. Based on prior literature, presumed overdose-related arrests were defined as those aged $\leq 50$ without an obvious traumatic, respiratory, or cardiovascular (e.g., STEMIs) etiology. TTM was carried out with a combination of the Arctic Sun device, refrigerated crystalloid/antipyretics (goal temperature $32-36{ }^{\circ} \mathrm{C}$ ), maintained for $24 \mathrm{~h}$. Variables included demographics, location (ED vs. OHCA), initial rhythm, ACLS medications (including 20\% fat emulsion), and initial return of spontaneous circulation (ROSC). The outcomes were in-hospital survival (primary), ED survival (secondary), and effects of ACLS medications (exploratory). Descriptive statistics, odds ratios (OR), 95\% confidence intervals (CI), and chi-squared were calculated with SPSSv20.

Results: Of 923 cardiac arrests in the study period, 195 cases were $\leq 50$ years, of whom there were 24 respiratory, 32 traumatic, and 5 cardiac etiologies, leaving $134(14.5 \%)$ patients for analysis (median age 41 years, $73.9 \%$ male, OHCA $89 \%$ ). The most common rhythm was asystole $(53.7 \%), 55$ patients $(41 \%)$ had ROSC, $35(26.1 \%)$ received TTM, and 14 survived to hospital discharge. TTM conferred increased odds of inhospital survival in the entire cohort (OR 9.5, CI 2.8-32.8, $p<0.001$ ) and in OHCA (OR 14.3, CI 2.9-72.0, $p<0.001$ ). In the ROSC subset, TTM conferred higher ED (OR 38.5, $p<0.001)$ and in-hospital survival (OR $1.6, p=\mathrm{NS})$. EMS calcium $(p=0.01)$ and atropine $(p=0.008)$ were associated with survival, while EMS dextrose was not (OR 0.2 , $p=0.032$ ). EMS naloxone had no association with $\mathrm{ED}$ (OR 1.6, $p=$ NS) or in-hospital survival (OR $0.7, p=\mathrm{NS}$ ). No patient receiving $20 \%$ fat emulsion survived $(0 / 4, p=\mathrm{NS})$.

Discussion: Interventions for OHCA with the best clinical effects were TTM, calcium, and atropine. Naloxone, dextrose, and 20\% fat emulsion had no benefit for the resuscitation of overdose-related cardiac arrest.

Conclusion: TTM was associated with improved in-hospital survival for overdose-related cardiac arrest.

\section{Loperamide-Induced Torsades de Pointes: A Case Series}

Kenneth Katz ${ }^{1}$, Robert Cannon ${ }^{1}$, Matthew Cook $^{1}$, Alexandra Amaducci ${ }^{1}$, Ryan Day ${ }^{1}$, Joshua Enyart ${ }^{1}$, Glenn Burket ${ }^{1}$, Jennifer Janssen ${ }^{2}$, Karl Williams ${ }^{2}$

${ }^{1}$ Lehigh Valley Health Network, Allentown, PA, USA

${ }^{2}$ Office of the Medical Examiner of Allegheny County, Pittsburgh, PA, USA

Background: Loperamide (Imodium ${ }^{\mathrm{TM}}$ ) is an antidiarrheal opioid acting at the $\mu$ receptor agonist in high concentrations. Due to the US opioid epidemic, loperamide abuse and ensuing life-threatening toxicity have surfaced. Hypothesis: The timing and duration of Torsades de Pointes (TdP) from loperamide toxicity is unpredictable and should be heeded with caution, even in asymptomatic patients and drug discontinuance.

Methods: Two patients presenting with life-threatening TdP requiring lengthy hospital admissions for confirmed loperamide toxicity are discussed. Patient data were retrieved from the hospital's EMR system.

Case \#1: A 28-year-old man with a history of depression and heroin abuse presented to the ED with shortness of breath and lightheadedness. He admitted to ingesting large amounts of loperamide daily. His initial ECG demonstrated sinus rhythm $67 \mathrm{bpm}$, right axis deviation, undetectable PR interval, QRS $168 \mathrm{~ms}$, and QTc $693 \mathrm{~ms}$. The patient was administered sodium bicarbonate and magnesium sulfate IV and admitted to the ICU. On HD 2, he developed multiple and persistent TdP. He was administered lidocaine and isoproterenol infusions, and a temporary, external pacemaker was placed for overdrive pacing. He was discharged in stable condition on HD 16 after all therapies were discontinued. Serum loperamide and desmethyl loperamide concentrations obtained on HD 1 measured 120 and $560 \mathrm{ng} / \mathrm{mL}$, respectively. Serum loperamide and desmethyl loperamide concentrations on HD 10 measured undetectable and $16 \mathrm{ng} / \mathrm{mL}$, respectively.

Case \#2: A 39-year-old woman with hepatitis C and a history of depression and IV drug abuse was transported to the ED after reported seizurelike activity. The patient experienced TdP in the ED and admitted to ingesting large amount of loperamide daily. An ECG demonstrated sinus rhythm $64 \mathrm{bpm}$, right axis deviation, PR interval $208 \mathrm{~ms}$, QRS interval $142 \mathrm{~ms}$, and QTc $687 \mathrm{~ms}$. She was administered IV magnesium, sodium bicarbonate, and, later, isoproterenol. After ICU admission, the patient experienced no further TdP and was discharged on HD 6. Loperamide and desmethyl loperamide concentrations on $\mathrm{HD} 4$ were undetectable and $27 \mathrm{ng} / \mathrm{mL}$, respectively.

Conclusion: These cases illustrate the extreme and persistent toxicity associated with loperamide abuse and need to treat ECG derangements aggressively, even in asymptomatic patients.

\section{2,4 Dinitrophenol Associated Hyperthermia Treated Successfully with Dantrolene}

Louise Kao, James Mowry

Indiana University, Indianapolis, IN, USA

Background: 2,4 Dinitrophenol (DNP) has well-known toxicity. Dantrolene has been successfully used in a single case report. We report another case of dantrolene use for DNP-associated hyperthermia.

Case Report: A 22-year-old previously healthy male was found confused and diaphoretic. Upon arrival to the emergency department, he related that $4 \mathrm{~h}$ prior he had taken a double dose of his bodybuilding supplements. Bottles provided included hydroxyzine, creatine, taurine, levothyroxine, and DNP.

On presentation, his initial vital signs were normal other than mild tachycardia at $113 \mathrm{bpm}$. He was confused, diaphoretic, and tremulous. The remainder of exam was unremarkable.

Initial laboratory evaluation were as follows: sodium $139 \mathrm{mmol} / \mathrm{L}$, potassium $4.1 \mathrm{mmol} / \mathrm{L}$, chloride $109 \mathrm{mmol} / \mathrm{L}, \mathrm{CO}_{2} 18 \mathrm{mmol} / \mathrm{L}$, BUN $34 \mathrm{mmol} / \mathrm{L}$, creatinine $2.11 \mathrm{mmol} / \mathrm{L}$, glucose $499 \mathrm{mg} / \mathrm{dL}$, TSH and T4 both in normal range, and creatine phosphokinase 456 units/L. 
Toxicology testing including ethanol, acetaminophen, aspirin, and urine drug screen containing amphetamine, methamphetamine, barb, benzo, cannabinoid, cocaine, opiate, and PCP were negative.

Eight hours after hospital arrival, he was increasingly disoriented with severe respiratory distress. His vital signs were T $38.6^{\circ} \mathrm{C}$; HR $113 \mathrm{bpm}$; BP 114/45; RR 63 breaths/min; and oxygen saturation $97 \%$ on $4 \mathrm{~L}$ of oxygen. He required intubation for respiratory failure. He was given dantrolene $180 \mathrm{mg}$ intravenously. Temperature reached a maximum of $40.0^{\circ} \mathrm{C}$. He received two additional doses of dantrolene over the next $10 \mathrm{~h}$ for a total of $7 \mathrm{mg} / \mathrm{kg}$. Hyperthermia resolved. He was extubated on hospital day 4 and discharged on hospital day 6 in good condition.

Discussion: DNP has had a rise in popularity due to internet availability. DNP causes uncoupling of oxidative phosphorylation and clinical effects include hyperthermia, tachycardia, diaphoresis, and in fatal cases cardiovascular collapse. Dantrolene has been successfully used in a single published case report to our knowledge. Dantrolene may ameliorate the hypermetabolic state in DNP toxicity by lessening excitation-contraction coupling in muscle cells and thus attenuate hyperthermia.

Conclusion: We present a case of DNP-associated hyperthermia, successfully treated with dantrolene in addition to good supportive measures. This case adds to the sparse existing published evidence for the use of dantrolene in DNP toxicity.

\section{The Blood Urea Nitrogen to Creatinine Ratio as a Novel Prognostic} Biomarker in Liver Failure Associated with Acetaminophen Toxicity

\section{Alexander Huh, Clifton Callaway, Anthony Pizon \\ University of Pittsburgh Medical Center, Pittsburgh, PA, USA}

Background: A common cause of acute liver failure (ALF) is acetaminophen (APAP) toxicity, and patients with severe ALF may require liver transplantation to avoid death. The Kings College Criteria has been developed for prognostication, but is fraught with many limitations, necessitating additional prognostic tools for a bad outcome in APAP toxicity. Studies have found that a compromised liver will result in decreased urea synthesis in the presence of acute kidney injury; therefore, we are investigating the value of the blood urea nitrogen to creatinine ratio (BUN/Cr) as a prognostic indicator of poor outcome in ALF after APAP toxicity.

Hypothesis: Patients who die or require liver transplantation from acetaminophen-induced hepatic toxicity will have significantly lower $\mathrm{BUN} / \mathrm{Cr}$ than patients whose livers spontaneously recover.

Methods: This is a retrospective study utilizing pharmacy records of $N$ acetylcysteine administration for APAP toxicity at a single academic quaternary care center from January 2005 to October 2012. A total of 230 patients were identified. Cases were excluded if the patient had preexisting liver or renal disease $(n=10)$ and if AST never exceeded $1000 \mathrm{IU} / \mathrm{L}(n=113)$. Patients were then divided based on outcome: death $(n=22)$, spontaneous survival $(n=73)$, or transplant $(n=12)$. BUN/Cr, along with other data (demographics and concurrent lab tests), was collected on a standardized data collection sheet. We compared individual laboratory values on each day between groups using ANOVA. We performed logistic regression to account for repeated measurements within subjects in order to test for independent associations of each variable with outcome.

Results: When comparing subjects with death or transplanted $(n=34)$ to subjects who survived ( $n=73)$, BUN/Cr did not differ (NS, ANOVA). However, $\mathrm{pH}(p<0.001$, ANOVA), phosphorus ( $p=0.012$, ANOVA), lactate ( $p<0.001$, ANOVA), and INR ( $p<0.001$, ANOVA) demonstrated significant difference between groups.

Discussion: There was a tendency for BUN/Cr to be lower in those who die or require transplantation; however, this was not statistically significant. We did observe changes in biomarkers previously found to indicate a poor outcome in APAP-induced ALF, identified in previous studies.

Conclusions: A lower BUN/Cr was not related to worse outcome for subjects with acute liver failure in setting of APAP toxicity.
38. A Retrospective Review of Epinephrine Exposures Reported to a Poison Center

Adrienne Hughes ${ }^{1}$, Matthew Valento ${ }^{1,2}$, Betty Chen ${ }^{1,2}$

${ }^{T}$ University of Washington Division of Emergency Medicine, Seattle, WA, USA

${ }^{2}$ Washington Poison Center, Seattle, WA, USA

Objectives: Epinephrine is a commonly used medication administered for life-threatening conditions. Unfortunately, drug delivery errors are common.

Methods: We performed a retrospective review of a poison center's database for cases of single-substance epinephrine exposures evaluated in a healthcare facility from 2006 to 2016. Data collected included age, context, clinical effects, interventions, and outcome.

Results: Fifty patients were identified with ages ranging from 8 months to 80 years. Unintentional exposure via auto-injectors occurred in 46 exposures. Three cases involved medical errors, and one case involved a suicide attempt. No therapy was required in $46 \%(n=23)$; there were no reported deaths.

In auto-injector exposures $(n=46)$, the most common injection location was the finger $(n=36,78 \%)$. Reported clinical effects were blanching/ pallor $(n=24,52 \%)$, puncture $(n=15,32.6 \%)$, pain $(n=9,19.5 \%)$, numbness $(n=8,17.3 \%)$, and tachycardia $(n=4,8.7 \%)$. No effects were reported in $19.5 \%(n=9)$. Warm soaks alone were used in $11(24 \%)$ cases. Pharmacologic treatment was used in $7(15 \%)$ cases including topical nitroglycerin $(n=3)$, local phentolamine injection $(n=3)$, and local bupivicaine injection $(n=1)$.

Three cases involved medical errors. (1) A 78-year-old man received $1 \mathrm{mg}$ of epinephrine intravenously for contrast-induced anaphylaxis; no systemic effects were reported. (2) An 8-month-old boy received 2 mg of IV epinephrine instead of inhaled epinephrine. He required intubation after developing tachycardia and agitation. (3) A 60-year-old woman received $3 \mathrm{mg}$ of IM epinephrine for an allergic reaction. She reported "jitteriness" but was later discharged home. A 28-year-old woman broke into a code cart and injected $1 \mathrm{mg}$ of epinephrine into her neck as a suicide attempt. She developed hypertension and tachycardia followed by hypotension, ischemic ECG changes, elevated troponin, pulmonary edema, and left ventricular dysfunction. She was admitted to the ICU and discharged 4 days later.

Conclusion: In our series, the majority of exposures occurred via autoinjector misadministration. Significant adverse effects were uncommon, and nearly half of all cases required no treatment. Despite being a highrisk medication, epinephrine dosing errors still occur, often in the setting of anaphylaxis. Significant adverse effects were more commonly reported following medical errors or intentional overdose.

39. Assessing Appropriate Pediatric Acetaminophen Dosing Given by Caregivers: A Cross-Sectional Study

\author{
Diane Hindman $^{1,2}$, Ingrid Anderson ${ }^{3,4}$, Haitham Haddad ${ }^{3,4}$ \\ ${ }^{T}$ Emory University, Atlanta, GA, USA \\ ${ }^{2}$ Georgia Poison Center, Atlanta, GA, USA \\ ${ }^{3}$ Case Western Reserve University, Cleveland, OH, USA \\ ${ }^{4}$ University Hospitals - Rainbow Babies and Children, Cleveland, $\mathrm{OH}$, \\ USA
}

Background: Acetaminophen is frequently given to children by wellmeaning caregivers. Dosing in children is fraught with challenges; previous study has identified that incorrect acetaminophen doses are highly likely. It remains the most commonly reported drug in accidental and intentional ingestions, and despite an effective antidote, the analgesic most often results in death. As an over-the-counter (OTC) medicine, it is widely available in numerous dosage formats and often disguised in combination products by brand marketers.

Hypothesis: More than $50 \%$ of caregivers inaccurately dose acetaminophen when treating pediatric fever. 
Methods: This cross-sectional observational study enrolled caregivers of children, from birth to 13 years old, who presented to an urban academic pediatric emergency department with fever between August 2015 and April 2016. Recruited subjects completed a standard questionnaire evaluating demographics of subjects and their children; subject understanding of acetaminophen dosing and hazards; and fever determination and management. The hospital's Institutional Review Board approved the study. Study data was entered and stored in the Research Electronic Data Capture (REDCap) database. Data was analyzed using descriptive statistics. No monetary funding support was received.

Results: One hundred forty-two subjects, predominantly young single black women from the local zip code, were enrolled. Their children were mainly black infants and toddlers younger than 3 years old. Overall reported home acetaminophen dosing ranged from 0.1 to $32 \mathrm{mg} / \mathrm{kg}$. Fortytwo percent were within the correct dosing range of $10-15 \mathrm{mg} / \mathrm{kg}$, while $46 \%$ were underdosed and $12 \%$ overdosed. $11.2 \%$ of caregivers could report the concentration of liquid acetaminophen administered. Less than half $(46.5 \%)$ of caregivers could identify serious risks of acetaminophen overdose.

Discussion: Despite OTC product labeling and educational efforts by healthcare providers, the majority of caregivers reported using safe, but often inappropriate and sometimes unsafe, acetaminophen dosing. Underdosing is unlikely to control fever and may lead to unnecessary healthcare visits. Overdosing, particularly if multiple doses are administered, may result in acetaminophen toxicity. Initiatives to improve health literacy and educate caregivers about safe and appropriate use of acetaminophen should continue.

Conclusions: In this single center observational study, less than $50 \%$ of caregivers reported providing appropriate acetaminophen doses when treating their children's fever.

\section{Analysis of Look-Alike/Sound-Alike Medication Errors in the Emergency Department}

Aaron Zach Hettinger, Katrina Smith, Allan Fong, Maryann MazerAmirshahi, Grace Tran, Erica Savage

MedStar Health, Washington, DC, USA

Background/Objective: Look-Alike/Sound-Alike (LA/SA) medication errors account for $25 \%$ of medication errors. We reviewed patient safety data related to LA/SA medication errors that occurred in the emergency department (ED) compared to other clinical areas in the healthcare system.

Study Hypothesis: ED LA/SA medication errors would account for a significant portion of errors and more severe outcomes than non-ED settings.

Methods: Patient safety event (PSE) data was reviewed using text mining and natural language processing algorithms to search for LA/SA-related medication errors. The PSE database contains roughly 90,000 incidents from self-reported near misses and harm events by frontline staff in an academic healthcare system. Each case was reviewed independently and categorized by stage in the medication-use system (documentation, ordering, dispensing, or administration) and error severity level (based on anticipated and actual patient outcomes). Comparisons between ED and non-ED datasets consisted of Fisher's exact test using a significance level of 0.05 .

Results: A total of 282 incidents met inclusion criteria for a LA/SA error, in which $37(13 \%)$ occurred in the ED. Error frequency by stage was $0 \%$ documentation, $22 \%$ ordering, $43 \%$ dispensing, and $5 \%$ administration in the ED versus $9,23,53$, and $15 \%$, respectively, in non-ED settings $(p=0.049)$. The most common LA/SA error pairs in the ED were oxycodone extended release-oxycodone immediate release $(11 \%)$, ceftriaxone-cefazolin (8\%), hydroxyzine-hydralazine $(8 \%)$, prednisoneprednisolone (8\%), and oxycodone-hydrocodone (5\%). In the ED, $49 \%$ of errors reached the patient versus $38 \%$ across the system (NS). Level of error severity did not differ between the ED which included $0 \%$ no effect,
$19 \%$ mild, $53 \%$ moderate, $11 \%$ severe, and $3 \%$ critical LA/SA errors versus $2,40,50,9$, and $0 \%$ in non-ED settings, respectively.

Discussion: While limited by a small retrospective dataset, the stages of LA/SA errors differed between the ED and non-ED settings. Our findings highlight the opportunity to improve medication processes during the administration and dispensing stages.

Conclusion: The nature of LA/SA errors differed in the ED compared to other clinical settings, necessitating further research to identify the contributing factors and develop mitigation strategies to improve medication administration and dispensing processes in the ED.

\section{Evaluation of Flumazenil Adverse Effects when Used in the Iatrogenic Benzodiazepine Delirious Patient: a retrospective study at one center}

Michelle Hieger $^{1,3}$, Kevin Maskell ${ }^{3,2}$, Brandon Wills ${ }^{3}$

${ }^{7}$ Wellspan Health, York Hospital, York, PA, USA

${ }^{2}$ Madigan Army Medical Center, Tacoma, WA, USA

${ }^{3}$ Virginia Commonwealth University, Richmond, VA, USA

Background: Since the introduction of flumazenil in 1987, there has been controversy regarding the potential risk in its administration.

Research Question: What is the incidence of adverse drug events when using flumazenil for patients with suspected iatrogenic benzodiazepine delirium?

Methods: This is a retrospective cross-sectional study over a 3-year period in a single academic center. Patients were included who were ordered both flumazenil and benzodiazepines during their hospitalization, had a diagnosis of delirium, and had toxicology service involvement. A single investigator abstracted all data. Adverse drug events were considered temporally associated with flumazenil use if they occurred within $1 \mathrm{~h}$ of administration. The Common Terminology Criteria for Adverse Events (CTCAE) v4.0 was used to assess the adverse events.

Results: Two hundred eighty-six of 501 patients met inclusion criteria: 189 males, 97 females. Benzodiazepines were administered for multiple conditions including benzodiazepine withdrawal, hepatic encephalopathy, and 43 with opiate overdose and/or withdrawal. Some had more than one diagnosis. Benzodiazepines were used chronically in 100 patients, 30 patients had a known seizure disorder or history of seizure, and 40 patients had a documented seizure during the hospitalization or just prior to arrival. Median total flumazenil dose was $1.5 \mathrm{mg}$ (IQR 0.5-3.5). Improved objective delirium parameters were identified in 242 patients after flumazenil, and 49 patients had no change. In intubated patients, $82 / 98$ were successfully extubated with flumazenil administration. Complications from flumazenil were noted in 10 patients. Of these, six were categorized grade 2 or grade 3 (moderate) on the CTCAE scale, including one self-limited seizure.

Discussion: This study was important to evaluate the use of flumazenil in patients who had suspected iatrogenic benzodiazepine delirium. This unique population all had toxicologist involvement in the flumazenil administration. Six patients $(2.1 \%)$ required intervention within an hour of flumazenil administration. In comparison, a naloxone review showed agitation/combativeness as an adverse drug effect in $7.9-15 \%$ of patients. Conclusion: Flumazenil can be safely administered in patients with suspected iatrogenic benzodiazepine delirium to evaluate for the cause of delirium and to assist in further treatment if needed. Flumazenil can also be used effectively in patients with iatrogenic benzodiazepine delirium to assist in extubation.

\section{Hydrogen Peroxide Exposures Reported to the Toxicology Investigators Consortium (ToxIC)}

Benjamin Hatten ${ }^{1,2}$, On Behalf of the Toxicology Investigators Consortium (ToxIC)

${ }^{1}$ Department of Emergency Medicine, University of Colorado School of Medicine, Aurora, CO, USA

${ }^{2}$ Rocky Mountain Poison and Drug Center, Denver, CO, USA 
Background: High concentration peroxide ingestions have been associated with embolic events and caustic injuries in case reports. However, little has been published regarding the epidemiology of peroxide ingestions.

Research Question: To compare the epidemiology of and treatment following household and high concentration peroxide exposures.

Methods: The Toxicology Investigators Consortium (ToxIC) Registry records all clinical consults by an international network of medical toxicologists in a standardized fashion. ToxIC was queried for all cases of household (less than or equal to $10 \%)$ or high concentration $(>10 \%)$ hydrogen peroxide ingestion or inhalation exposures. Household and high concentration exposures were compared with regard to demographics, associated signs and symptoms, evaluation, and treatment expressed with descriptive statistics, tests of proportions, chi-squared tests, and Woolf's adjusted odds ratio.

Results: Thirty-nine hydrogen peroxide exposures, 11 high concentration and 28 household, were reported to ToxIC from 2010 to 2016. Mean age of household exposures was 37 years vs 51 years for high concentration. In cases describing the amount ingested, the mean volume of high concentration peroxide was $52 \mathrm{ml}$. High concentration peroxide exposures were more likely to exhibit CNS toxicity (OR 15.4; 95\% CI 1.5-160.8) and GI/hepatic toxicity (OR 7.4; 95\% CI 1.1-49.2). Weakness/paralysis was reported in $2 / 11(18 \%$; $95 \%$ CI $2-52 \%)$ high concentration peroxide exposures and no household exposures. $5 / 11$ (45\%; 95\% CI 15-75\%) of high concentration peroxide exposures reported portal venous gas on abdominal CT. 7/11 high concentration and 1/28 household exposures were treated with hyperbaric oxygen therapy (OR 47.3; 95\% CI 4.5492.3). No deaths were reported.

Discussion: Exposures contained in the ToxIC registry are limited to direct consults by a medical toxicologist and may represent a more severe spectrum of disease compared to the total population of exposures. Effectiveness of treatment and long-term outcomes aside from death are not available from upper-level ToxIC data, limiting the evaluation of hyperbaric oxygen therapy as a therapy following peroxide ingestions.

Conclusion: In cases recorded in the ToxIC registry, high concentration peroxide exposures exhibit more severe toxicity and are more frequently treated with hyperbaric oxygen compared to household concentrations.

\section{$\neg 7$ This research was performed in collabo- ration with the ACMT Toxicology Investigators Consortium. \\ Toxicology Investigators consortium}

\section{A Case of Bupropion-Induced Non-Convulsive Status Epilepticus}

Stephen Harding ${ }^{1,2}$, Danny Lugassy ${ }^{1,2}$, Howard Greller ${ }^{3}$

${ }^{T}$ Division of Medical Toxicology, Ronald O. Perelman Department of Emergency Medicine, NYU School of Medicine, New York, NY, USA

${ }^{2}$ New York City Poison Control Center, New York, NY, USA

${ }^{3}$ Division of Medical Toxicology, Department of Emergency Medicine, SBH Health System - St Barnabas Hospital, Bronx, NY, USA

Case Report: A 32-year-old male with a history of depression and systemic lupus erythematosus presented to the emergency department after an intentional overdose of between 30 and 90 tablets (based on pill count) of his 300-mg bupropion XL. Prior to arrival, EMS reported two generalized tonic-clonic seizures, followed by another shortly after presentation, and he was subsequently intubated. Initial vital signs were BP 138/ $62 \mathrm{mmHg}$; HR 145 beats/min; RR 18/min (ventilator); and $\mathrm{O}_{2}$ 94\%. His initial EKG showed sinus tachycardia at 148 beats/min, with a QTc of $549 \mathrm{~ms}$. Gastric lavage was performed, without return of discrete pill fragments, and whole bowel irrigation with polyethylene glycol was initiated after administration of activated charcoal. He was noted to have fixed and dilated pupils, without motor activity. He was given a total of
$12 \mathrm{mg}$ of lorazepam and then placed on infusions of fentanyl and propofol. Neurology was consulted and a bedside EEG was performed which showed numerous bursts of generalized epileptiform activity and electrographic temporal seizures. He was given a $0.2-\mathrm{mg} / \mathrm{kg}$ loading dose of midazolam and $5 \mathrm{~g}$ of pyridoxine, with continued titration of the propofol, but no clinical change was observed. Phenobarbital loading was initiated and he was transferred to a tertiary care center. Upon arrival at the receiving hospital, he no longer exhibited seizure activity on EEG, although on physical exam his pupils continued to be fixed and dilated and he demonstrated no corneal or gag reflexes. Over the course of several days, he regained reflexes and began to make purposeful movements, without recurrent seizure activity. He had a prolonged hospital course, attributed to aspiration pneumonia. Five days post presentation, his serum bupropion concentration (drawn at time of transfer) returned at greater than $400 \mathrm{ng} / \mathrm{mL}$ (reference range $50 \mathrm{ng} / \mathrm{mL}$ ). Levels were not trended by the primary team.

Conclusions: We present a case of bupropion-related non-convulsive status epilepticus, associated with high serum concentrations refractory to several therapeutic interventions. Given the ubiquity of prescription bupropion for multiple indications, this concerning consequence of overdose should be included in the differential diagnosis of an unresponsive patient.

\section{A Novel Tool to Assess Impairment from Alcohol in Emergency Department Patients- the HII score}

\author{
Jason Hack ${ }^{1}$, Eric Goldlust ${ }^{2}$, Dennis Ferrante ${ }^{1}$, Natalija Farrell ${ }^{1}$, Brian \\ Zink \\ ${ }^{1}$ Brown University, Providence, RI, USA \\ ${ }^{2}$ Santa Clara Hospital, Kaiser Permanente, Santa Clara, CA, USA
}

Background: Emergency departments (EDs) care for over 35 million alcohol-impaired (AI) patients annually. There is no standard ED assessment of AI patients. We sought to evaluate a novel standardized assessment of AI-Hack's Impairment Index (HII score).

Methods: A retrospective chart review was performed for all AI patients seen in our busy urban ED over 24 months. Trained nurses evaluated AI patients with both "usual" and HII testing every $2 \mathrm{~h}$. Patients were stratified by frequency of visits for AI during this time: high ( $\geq 6$ ), medium (2-5), and low (1). Within each category, comparisons were made between HII scores, measured ethanol levels, and healthcare providers' (HCPs') general assessment of AI. Changes in HII scores over time were also evaluated.

Results: Eight thousand seventy-four visits from 3219 unique patients were eligible for study, including 7973 (98.7\%) with ethanol levels, 5061 (62.7\%) with complete HII scores, and 3646 (45.2\%) with HCP assessments. Correlations between HII scores and ethanol levels were poor (Pearson's $R^{2}=0.09,0.09$, and 0.17 for high-, medium-, and lowfrequency strata). HII scores were excellent at discriminating HCPs' assessment of AI, while ethanol levels were less effective. Omitting extrema, HII scores fell consistently by an average 0.062 points per hour, throughout patients' visits.

Conclusions: The HII score use applied a quantitative, objective assessment of alcohol impairment. HII scores were superior to ethanol levels as an objective clinical measure of impairment. The HII declines in a reasonably predictable manner over time, with serial evaluations corresponding well with HCP evaluations.

\section{User Self Report and Detection of Clandestine Fentanyl among Adult Heroin Users}

Matthew Griswold ${ }^{1}$, Peter Chai $^{1}$, Brittany Chapman ${ }^{1}$, Melissa Friscia ${ }^{2}$, Alexander Krotulski ${ }^{2}$, Edward Boyer ${ }^{1}$, Barry Logan ${ }^{2}$, Kavita Babu ${ }^{1}$

${ }^{1}$ University of Massachusetts School of Medicine, Worcester, MA, USA

${ }^{2}$ Center for Forensic Science Research and Education, Willow Grove, PA, USA

Background: The adulteration of heroin with clandestine fentanyl is one suspected etiology for the increase in heroin-related overdose deaths. 
Normally, an efficient method of assessing dimensions of drug use, selfreport may be inaccurate because heroin users are frequently unaware of the fentanyl adulteration. In this study, we compared heroin user selfreport with gold standard urine testing.

Research Question: Can heroin users accurately self-report fentanyl exposure in the setting of overdose?

Methods: Adult heroin users presenting to an urban, tertiary care ED after overdose were eligible for enrollment if they had received naloxone and were able to provide verbal consent in English. Subjects underwent a semi-structured interview and a urine specimen was collected. Results were evaluated using descriptive statistics. This study was approved by the Institutional Review Board.

Results: Between August and October 2016, 20 adult heroin users who presented after overdose were enrolled in a convenience sample; two subjects declined to participate. Average age was 31,50\% identified as female. Users reported injection heroin use (12/20), intranasal use (7/20), and both (1/20). Two subjects denied possible fentanyl exposure, while $6 /$ 20 remained uncertain. Suspicion of fentanyl exposure was reported by $12 / 20$ participants, with qualitative reports of change variations in the effects, texture, and color of the drug. No patients reported pharmaceutical fentanyl use or attempts to purchase fentanyl. Urine testing revealed the presence of fentanyl (or metabolites) in $20 / 20$ of cases, with an absence of heroin metabolites in $2 / 20$ cases.

Discussion: All enrolled heroin overdose individuals in this study had urine liquid chromatography time-of-flight mass spectrometry screens positive for fentanyl. Individual self-report of suspected fentanyl contamination did not correlate with urine testing. In this small sample, we found universal presence of fentanyl in heroin users presenting after an overdose requiring naloxone. Limitations of this pilot study include the small sample size, convenience sampling, and regional/temporal variation of heroin adulterants.

Conclusion: In this pilot study, the presence of fentanyl was detected in $100 \%$ of heroin overdose individuals presenting to the $\mathrm{ED}$ after receiving naloxone, with only $60 \%$ agreement between user self-report of fentanyl exposure and the identification of fentanyl.

\section{It's Just Math - Unless It's Toxic!}

Elisabeth Giblin, Michele Zell-Kanter Cook County Hospital, Chicago, IL, USA

Background: Dosing errors from erroneous pharmaceutical calculations result in morbidity and mortality.

Hypothesis: Emergency medicine (EM) residents perform poorly calculating correct doses.

Methods: A 6-question needs assessment survey of EM residents was conducted at a city-wide conference. Residents performed simple pharmaceutical calculations and were queried regarding resources they typically use.

Results: Fifty-one EM residents completed the survey; PGY1 $n=29$, PGY $2 n=14$, PGY $3 n=8$. The majority of PGY2 and PGY3 residents performed similarly having the correct answer on at least 4/6 questions. Only $52 \%$ of PGY1 responded correctly to at least $4 / 6$ questions. Sixtyfive percent of PGY1, 50\% of PGY2, and 75\% of PGY3 residents thought it was very important to correctly perform basic calculations. Google $\AA$ and Up To Date ${ }^{\circledR}$ were frequently used to assist with calculations; $45 \%$ of PGY1, $50 \%$ of PGY2, and $37 \%$ of PGY3 residents rely on smartphone apps. More than $70 \%$ of residents in all 3 years utilize clinical pharmacists (Pharm.D.) for calculating doses. Pharm.D. services were available at all times in the majority of survey responses.

Discussion: The trends from this survey are valuable despite the sample size. Residents performed poorly when calculating doses. This may contribute to patient morbidity and mortality. Internet resource use increased as year of training increased. Pharm.D. services are extensively utilized in all 3 years of training.

Conclusion: Residents and patient outcome would benefit from additional resident training in calculating correct dosages.
47. A Case of Intrathecal Digoxin Administration: Disaster in the Delivery Room

\author{
Eddie Garcia $^{1}$, Diane Calello ${ }^{1,2}$, Bruce Ruck ${ }^{1,2}$, Lewis Nelson ${ }^{1,2}$ \\ ${ }^{T}$ Rutgers NJMS, Newark, NJ, USA \\ ${ }^{2}$ New Jersey Poison Information and Education System, Newark, NJ, USA
}

Background: Digoxin is a cardioactive steroid with a narrow therapeutic index. Digoxin has an extensive historical involvement in medical errors but there is only one prior report of intrathecal digoxin administration.

Case: A 36-year-old woman undergoing a high-spinal block during a caesarian section for twin gestation had an inadvertent intrathecal administration of $375 \mathrm{mcg}$ of digoxin. The patient was unresponsive immediately after the procedure and was intubated without the need for sedation. The patient's mental status improved, and, by day 6 , the patient was extubated. Initially, the patient had no motor function below T10 but regained weak plantar and hip flexion by day 7 . Magnetic resonance imaging on day 7 showed neuronal injury from T3 to the conus medullaris consistent with ischemia, demyelination, or myelitis. Repeat MRI on day 10 showed injury only from T4 to T9. The serum digoxin level at $2 \mathrm{~h}$ was $0.8 \mathrm{ng} / \mathrm{mL}$ and trended downwards. She was given multiple doses of Digibind, starting on day 6.

A preliminary error investigation revealed a stocking error, as a vial of digoxin was placed where lidocaine should have been. The patient is still in rehabilitation with profound weakness in her lower extremities. Although her mental status is near baseline, she continues to have intermittent episodes of disorientation.

Discussion: Intrathecal digoxin administration resulted in severe paresis. Bagherpour et al. reported three cases of accidental intrathecal administration of digoxin presenting as paralysis, parasthesias, and hyporeflexia in the lower limbs. However, in all cases, symptoms resolved within $24 \mathrm{~h}$. They proposed that high levels of digoxin may non-selectively inhibit neuronal sodium channels causing temporary paralysis. In addition to this mechanism, we postulate that digoxin or one of the excipients in the preparation caused prolonged vasoconstriction, resulting in neuronal ischemia and more lasting symptomatology.

Conclusion: Severe neuronal injury may result from intrathecal digoxin exposure.

\section{Serial Nonsense? Clinical Characteristics and Trends in Cases With Serial Carboxyhemoglobins}

Ryan Gallagher ${ }^{1}$, Daniel Gallagher ${ }^{1}$, Rachel Castelli ${ }^{1}$, Nicholas Hosey ${ }^{1}$, Eric Macrae $^{1}$, Erin Lawson ${ }^{1}$, Erin Willard ${ }^{1}$, Evan Slanzcka ${ }^{1}$, Jeremy Hunter ${ }^{1}$, Mark Scott ${ }^{1}$, Stephen Thornton ${ }^{1,2}$

${ }^{1}$ University of Kansas Hospital Department of Emergency Medicine, Kansas City, KS, USA

${ }^{2}$ University of Kansas Hospital Department of Medical Toxicology, Kansas City, KS, USA

Background: Carboxyhemoglobin (COHgb) levels are commonly obtained when there is suspicion for carbon monoxide exposure though their true prognostic value remains controversial. For unclear reasons, serial $\mathrm{COHgb}$ levels are sometimes obtained despite the well-established halflife of $\mathrm{COHgb}$ with various degrees of oxygen supplementation. We sought to evaluate the clinical characteristics and trends when serial carboxyhemoglobin levels were obtained.

Methods: A retrospective review of all patients with at least one $\mathrm{COHgb}$ level between $4 / 2010$ and $4 / 2015$ at a quaternary care academic medical center was performed. All charts were individually reviewed and interrater reliability assured through training and evaluation of Kappa scores

Results: The search identified 624 cases. A total of 106 (17\%) of these cases had multiple carboxyhemoglobin levels obtained. The mean number of $\mathrm{COHgb}$ levels obtained was 2.6 (range 2-9). The average initial 
level was $8.9 \%$, subsequent levels averaged $2.8 \%$ at $353 \mathrm{~min}, 1.8 \%$ at $663 \mathrm{~min}$, and $1.1 \%$ at $1095 \mathrm{~min}$. The longest interval was $3807 \mathrm{~min}$ (2.6 days) to obtain a total of four levels. The serial $\mathrm{COHgb}$ group was more likely to be admitted to the ICU, have a burn injury, and receive hyperbaric oxygen therapy. No significant difference was found in age, gender, rate of smoking, initial COHgb level, or mortality. A total of four patients were identified as having a change in carboxyhemoglobin level from normal (defined as $<2 \%$ by the institution lab) to abnormal on serial levels. The largest interval increase was $1.9 \%$. All four patients were current smokers, none of their subsequent carboxyhemoglobin levels exceeded $4 \%$ at any time, and there were no deaths.

Conclusion: Ordering of serial carboxyhemoglobin levels occurred in $17 \%$ of cases of which four patients $(3.8 \%)$ were observed to increase from normal to abnormal carboxyhemoglobin levels based on the laboratory standard; however, the clinical significance of this increase is questionable. Despite being more likely to have a burn injury, be admitted in the ICU, and receive hyperbaric oxygen therapy, there was not a statistically significant difference in mortality. This study presents evidence that there is minimal value in obtaining serial carboxyhemoglobin levels and the practice should be discouraged.

\section{Accidental Flibanserin Poisoning in a Child Resulting in CNS Depression and Intubation for Airway Protection}

Benjamin Willenbring, Christian Lintner, Stacey Bangh, Jon Cole Minnesota Poison Control System, Minneapolis, MN, USA

Background: Flibanserin (Addyi ${ }^{\circledR}$ ) was approved in 2015 by the US Food and Drug Administration for the treatment of adult women with acquired generalized hypoactive sexual desire disorder. The approved dose is $100 \mathrm{mg}$ by mouth once daily. Flibanserin is known to cause CNS depression, hypotension, and syncope, and a Risk Evaluation and Mitigation Strategy (REMS) post market surveillance program to monitor these effects is in place. In vitro, flibanserin activates $5-\mathrm{HT}_{1 \mathrm{a}}$ and antagonizes 5- $\mathrm{HT}_{2 \mathrm{a}}, 5-\mathrm{HT}_{2 \mathrm{~b}}, 5-\mathrm{HT}_{2 \mathrm{c}}$, and $\mathrm{D}_{4}$ receptors. To our knowledge, no published case reports of flibanserin poisoning in children exist in the medical literature.

Hypothesis: Accidental flibanserin poisoning in children is associated with CNS depression.

Methods: This is a single-patient chart review. An otherwise healthy 2-year-old boy in his usual state of health was brought to the local emergency department after swallowing as many as seven 100 -mg tablets of flibanserin approximately $2 \mathrm{~h}$ prior to arrival. The mother reported the child was somnolent and ataxic at home after ingestion.

Results: In the emergency department, the child's pulse was 120 beats/min; his systolic blood pressure was $110 \mathrm{mmHg}$. Physical examination revealed spontaneous myoclonic movements and ataxia followed by lethargy. The boy tolerated a nasal airway. Prior to tertiary care center transfer, the child was intubated for worsening mental status (Glasgow Coma Scale 6). The child developed a fever to $100.8^{\circ} \mathrm{F}$ with radiographic evidence of pneumonia, concerning for aspiration. He was extubated on hospital day 3 and discharged home with no sequelae.

Discussion: The degree of CNS depression experienced in flibanserin poisoning is unknown as there are few, if any, published overdoses in the literature. This child developed evidence of aspiration pneumonia after lethargy suggesting the degree of CNS depression was clinically significant. The effects of flibanserin overdose in children are unknown; a minimum toxic dose is also unknown. This case demonstrates that even a small number of tablets is dangerous to a child.

Conclusion: Accidental flibanserin poisoning in children may result in serious CNS depression and subsequent intubation for airway protection.
50. The Expansion of Web-Based Learning in Medical Toxicology: The ACMT Experience

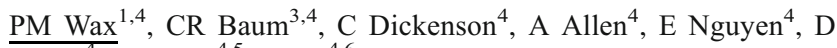
Turner $^{4}$, L Nelson ${ }^{4,5}, \mathrm{~S} \mathrm{Aks}^{4,6}$

${ }^{1}$ UT Southwestern Medical School, Dallas, TX, USA

${ }^{2}$ Yale University School of Medicine, New Haven, CT, USA

${ }^{3}$ University of Connecticut School of Medicine, Farmington, CT, USA

${ }^{4}$ American College of Medical Toxicology, Phoenix, AZ, USA

${ }^{5}$ Rutgers University, New Brunswick, NJ, USA

${ }^{6}$ University of Illinois, Chicago, IL, USA

Background: With the growing demand for online education, over the last few years, ACMT began offering web-based learning through several different modalities, including live and on-demand webinars, and on-demand eLearning modules. In 2011, ACMT established its monthly National Case Conference webinar series. Additional webinar series including the National Journal Club and ACMT Grand Rounds, and the 12-part DHSsponsored Chemical Agents of Opportunity series, were added to this online program in subsequent years. In 2014, ACMT began to co-manage the ATSDR/EPA funded Pediatric Environmental Health Specialty Unit (PEHSU) program. PEHSU produced webinars and eLearning modules have continued to expand ACMT's web-based offerings.

Hypothesis: Can the development of an online education program in medical toxicology engage health care professionals (HCPs) on an ongoing basis?

Methods: Since 2011, ACMT has tracked the number of webinar and eLearning module offerings per year and the number of HCPs who have availed themselves of this online education. The total number of call-in phone lines for each webinar have been tracked. Since in some cases there are multiple people participating from one phone line, beginning in 2014, ACMT began to poll each call-in line to assess the total number of listeners on each phone line. Webinars from both ACMT and PEHSU are also recorded and offered on-demand via the ACMT website or the PEHSU National Classroom (pehsunationalclassroom.org). Those accessing these on-demand webinars and eLearning modules in the PEHSU National Classroom have been tracked.

Results: ACMT began hosting 12 webinars, attended by at least 633 HCPs based on the number of call-in lines in 2011 and increased to 39 web-based activities (multiple webinar series and eLearning modules) with 4411 attendees by 2016 .

Conclusion: The development of an online learning program has facilitated additional and accessible education for HCPs throughout the past several years. ACMT has seen a substantial increase in the number of web-based learning activities and participants from 2011 to 2016.

\section{TOPEHSU

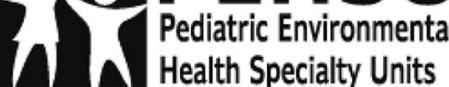

This presentation was supported by the ACMT Pediatric Environmental Health Specialty Unit (PEHSU) network. The ACMT PEHSU network is funded (in part) by the cooperative agreement FAIN: U61TS000238 from the Agency for Toxic Substances and Disease Registry (ATSDR).

51. Variation in Treatment Recommendations by US Poison Control Centers for Scorpion Envenomations

David Watts $^{1,2}$, A. Min Kang ${ }^{1,2}$, Daniel Brooks ${ }^{1,2}$

${ }^{I}$ Center for Toxicology, Pharmacology Education \& Research, University of Arizona College of Medicine, Phoenix, AZ, USA

${ }^{2}$ Banner - University Medical Center, Phoenix, AZ, USA 
Background: Scorpion stings accounted for more than 15,000 calls to US poison control centers (PCCs) in 2015. Numerous scorpion species are native to the southern US but the main one of medical concern is the Arizona bark scorpion (Centruroides sculpturatus). Although severe systemic effects are possible, local skin findings are rare. However, other native scorpion species can cause temporary local skin reactions. While retrospectively analyzing scorpion exposures reported to US PCCs, considerable variation in treatment recommendations for antibiotics and steroids were noted.

Methods: Scorpion envenomations reported to the American Association of Poison Control Centers' National Poison Data System from 2005 to 2015 were studied. A total of 185,402 records were analyzed for recommendations for antibiotics and steroids and were compared to reports of skin effects (e.g., edema and erythema). Only states with greater than 100 envenomations per year were included.

Results: There was wide variation in PCC recommendations for antibiotics and steroids. Arizona PCCs rarely recommended antibiotics $(0.1 \%)$ or steroids $(0.0 \%)$. In contrast, antibiotic recommendations in other states ranged from $0.6 \%$ (Georgia) to $44.9 \%$ (New Mexico) while steroid recommendations ranged from $0.1 \%$ (California) to $28.4 \%$ (Florida). Local dermal effects were rare in Arizona, with only $0.9 \%$ of calls recording edema or erythema. In other states, local effects were more prevalent: edema ranged from $5.5 \%$ (California) to $19.5 \%$ (Alabama) and erythema ranged from $6.6 \%$ (California) to $41.2 \%$ (Alabama).

Discussion: Scorpion envenomations reported to Arizona PCCs are rarely coded with local dermal effects and routinely do not result in recommendations for antibiotics or steroids. However, antibiotics and steroids are recommended more frequently in other states, where edema and erythema were also more common. There does not seem to be a strong correlation between these recommendations and clinical findings. Since local reactions to native scorpion stings are selflimited, it is unknown if these interventions are indicated or change patient outcome.

Conclusion: There is wide geographic variation in PCC recommendations for antibiotics and steroids following scorpion envenomation in the US. Further study may help elucidate the rationale for these recommendations.

52. Delayed Transition of Urine Drug Immunoassay from Negativity to Positivity in a Toddler with Exploratory Ingestion of Methadone

Parag Vora ${ }^{1}$, Ryan Surmaitis ${ }^{2,3}$, Joan Nolan ${ }^{3}$, Margueritte Pacholski ${ }^{3}$, Tracey Polsky ${ }^{1}$, Kevin Osterhoudt ${ }^{1,3}$

${ }^{T}$ The Children's Hospital of Philadelphia, Philadelphia, PA, USA

${ }^{2}$ Drexel University College of Medicine, Philadelphia, PA, USA

${ }^{3}$ The Poison Control Center at CHOP, Philadelphia, PA, USA

Background: Exploratory ingestion of prescription opioids can be dangerous to young children. Hospitalizations for opioid poisoning among children have increased over the past decade. There is uncertainty among emergency healthcare providers regarding the appropriate length of time to keep an asymptomatic young child under medical observation after a possible methadone ingestion, and there is uncertainty regarding the role of urine drug test results in decisionmaking.

Case Report: A 16-month-old boy gained access to his grandfather's pill bottle containing two $10-\mathrm{mg}$ methadone tablets and one lorazepam at 17:30. His mother found the lorazepam and one of the methadone tablets untouched, and one methadone table was swept "mostly intact" from his mouth. A urine sample was obtained from the boy at $7 \mathrm{~h} 20$ min after the exposure: it was qualitatively negative for methadone on the Vitros 5600 system (Ortho Clinical Diagnostics) at a threshold concentration of $150 \mathrm{ng} / \mathrm{mL}$ (quantitative value $112 \mathrm{ng} /$ $\mathrm{mL}$ ). The immunoassay was repeated using a urine sample collected $16 \mathrm{~h}$ post-exposure, and this sample tested qualitatively positive (quantitative value $361 \mathrm{ng} / \mathrm{mL}$ ). Presence of methadone in the urine was confirmed using gas chromatography/mass spectroscopy. The boy remained asymptomatic and well.

Discussion: In therapeutic doses ingested by adults, methadone has a bioavailability of $40-90 \%$ and peak plasma concentrations are reached between 1 and $7.5 \mathrm{~h}$. There is a concern that peak respiratory depression may occur later than peak analgesia. Methadone undergoes biotransformation and is eliminated by renal and fecal routes; the Vitros system tests for methadone in urine. We present a case demonstrating that a young child may have a negative urine drug immunoassay at $7 \mathrm{~h}$, using a reporting threshold of $150 \mathrm{ng} / \mathrm{mL}$, that will subsequently become positive. Once again, grandparent medication in containers bypassing child-resistant closures is noted as a risk factor for pediatric poisoning injury.

Conclusion: Poison control centers and emergency providers are cautioned against using urine methadone immunoassay results, with samples obtained within $7 \mathrm{~h}$ of ingestion, to "medically clear" exposed pediatric patients. The test performance characteristics, and optimal threshold values, in this setting merit further elucidation.

\section{Too Much NRG-3: Refractory Excited Delirium Associated with 2-(Methylamino)-1-(Naphthalen-2-yl)Pentan-1-one Use}

Stephen Thornton $^{1}$, Travis Langner ${ }^{2}$, Axel Adams ${ }^{3}$, Eric Vaughan ${ }^{5}$, Roy Gerona ${ }^{4}$.

${ }^{I}$ University of Kansas Hospital Poison Control Center, Kansas City, KS, USA ${ }^{2}$ University of Kansas School of Medicine, Department of Pediatrics, Division of Pediatric Critical Care, Kansas City, KS, USA

${ }^{3}$ University of California-San Francisco, School of Medicine, San Francisco, CA, USA, ${ }^{4}$ University of California -San Francisco, Division of Laboratory Medicine, San Francisco, CA, USA

${ }^{5}$ University of Kansas Hospital, Department of Emergency Medicine, Kansas City, KS, USA

Background: 2-(Methylamino)-1-(naphthalen-2-yl)pentan-1-one (NRG3 ) is a substituted cathinone that is advertised as a novel psychoactive substance (NPS). There is no published literature on its toxicity.

Hypothesis: NRG-3 use may be associated with severe excited delirium. Methods: This is a single patient chart review. A 15-year-old male with history of ADHD asthma became violent, vomited, and may have had a seizure after returning to his home from a store. Police were called and a conducted electrical weapon was deployed on him five times without effect. He was physically restrained and given a total of $10 \mathrm{mg}$ of midazolam IM by EMS without improvement. Upon arrival to the ED, he remained agitated and diaphoretic with pulse of $157 \mathrm{bpm}$, a blood pressure of $138 / 72 \mathrm{mmHg}$, and temperature of $37.2^{\circ} \mathrm{C}$. Over the next $5 \mathrm{~h}$, he was given haloperidol $10 \mathrm{mg}$ IV, ketamine $100 \mathrm{mg}$ IV, diphenhydramine $25 \mathrm{mg}$ IV, lorazepam $6 \mathrm{mg}$ IV, and phenobarbital $60 \mathrm{mg}$ IV but remained agitated. He was then intubated and sedated with propofol and fentanyl infusions. Laboratory evaluation was significant for serum creatinine of 1.28, CK of 12,279 U/L, and a UDS negative for amphetamines and cocaine. A CT head was negative. He was admitted and his vital signs normalized. He was extubated on hospital day 1 but remained anxious throughout his hospitalization. His creatinine and CK normalized over his 4-day hospitalization. He was discharged to an inpatient psych facility for management of depression.

Results: Serum and urine samples were obtained upon admission and analyzed by liquid chromatography time-of-flight mass spectrometry (TOF 6230, LC 1260, Agilent) using a library of 550 drugs including 285 novel psychoactive substances. NRG-3 was detected in urine and serum samples at concentrations of 109 and $10.2 \mathrm{ng} /$ $\mathrm{mL}$, respectively.

Discussion: Substituted cathinones are a large class of NPS associated with sometimes severe sympathomimetic effects. Human toxicity from NRG-3 has not previously been described. In this analytically confirmed case, it was associated with a refractory excited delirium. 
Conclusion: Health care providers should be aware of the potential for NRG-3 to cause severe excited delirium.

\section{Tryptamine Trauma! N,N-Dipropyltryptamine Associated Fall, Seizure and Rhabdomyolysis}

Stephen Thornton ${ }^{1}$, Travis Langner ${ }^{2}$, Roy Gerona ${ }^{3}$

${ }^{T}$ University of Kansas Hospital Poison Control Center, Kansas City, KS, USA

${ }^{2}$ University of Kansas School of Medicine, Department of Pediatrics, Division of Pediatric Critical Care, Kansas City, KS, USA

${ }^{3}$ University of California-San Francisco, Department of Laboratory Medicine, San Francisco, CA, USA

Background: N,N-Dipropyltryptamine is a potent hallucinogen whose use may be increasing but there is a paucity of literature regarding its toxicity.

Hypothesis: N,N-Dipropyltryptamine use may predispose patients to seizures and rhabdomyolysis.

Methods: This is a single patient chart review. A 17-year-old male with history of ADHD and opioid abuse was noted by family to be hallucinating and then became agitated and violent after smoking "marijuana." $\mathrm{He}$ had a physical altercation, fell, and struck his head. He began having seizure-like activity. EMS was called and administered midazolam $5 \mathrm{mg}$ IV which stopped the seizures. On arrival in the ED, he was unresponsive and endotracheally intubated. He had a pulse of $169 \mathrm{bpm}$, blood pressure of $128 / 59 \mathrm{mmHg}$, and temperature of $98.6^{\circ} \mathrm{F}$. He had multiple abrasions to his upper extremities and a scalp laceration. Laboratory evaluation showed an initial $\mathrm{pH}$ of 6.96 , leukocytosis of $25.6 \mathrm{~K} / \mathrm{UL}$, serum creatinine of 1.75 , and CK of $404 \mathrm{U} / \mathrm{L}$. He was admitted to the trauma intensive care unit. He was extubated $2 \mathrm{~h}$ later but became agitated and confused. This persisted for the next 4 days and required lorazepam and haloperidol along with dexmedetomidine infusion for sedation. He was found to be in rhabdomyolysis and his CK peaked at 83,000 U/L $48 \mathrm{~h}$ after admission and remained elevated upon discharge. He was discharged after 6 days and had a normal CK and creatinine 2 weeks later. Results: Admission serum and urine were obtained and analyzed by liquid chromatography time-of-flight mass spectrometry (TOF 6230, LC 1260, Agilent) using a library of 550 drugs including 285 novel psychoactive substances. N,N-Dipropyltryptamine was detected in both serum and urine at concentrations of 1740 and $971 \mathrm{ng} / \mathrm{mL}$, respectively. Discussion: N,N-Dipropyltryptamine is a rarely encountered tryptamine with no published human toxicity. Other tryptamines, such as 5-methoxyN,N-diisopropyltryptamine, have been reported to cause similar toxicity, including rhabdomyolysis. However, seizures have not previously been described but may be confounded by the traumatic head injury in this case. Conclusion: Health care providers should be aware of $\mathrm{N}, \mathrm{N}$ dipropyltryptamine's potential association with seizures and rhabdomyolysis.

\section{Highs in Stock? Inventory of Research Chemical Internet Vendors}

Stephen Thornton $^{1}$, Micheal Darracq ${ }^{3}$, Hallam Gugelmann ${ }^{2}$, Patil Armenian $^{3}$

${ }^{1}$ University of Kansas Hospital Poison Control Center, Kansas City, KS, USA

${ }^{2}$ Univerisity of California-San Francisco, Department of Emergency Medicine, San Francisco, CA, USA

${ }^{3}$ University of Californai-San Francisco-Fresno, Department of Emergency Medicine, Fresno, CA, USA

Background: Novel psychoactive substances (NPS) are commonly sold as research chemicals (RC) via the internet. There are few studies examining the inventory of these RC internet vendors (RCIVs).

Hypothesis: The inventory of RCIVs will be large and dynamic.

Methods: This was a prospective observational study. RCIVs were identified using a Google search term "Research Chemical Buy" performed
1 week prior to the study start date. The top 12 consecutive working sites were included in the study. Sites that had a broken URL, closed permanently, or were for body building compounds only were excluded. Inventory was taken of all single substance, non-brand name RCs and the sites re-inventoried every week for 12 weeks. Newly listed RCs and those that disappeared from the websites were documented. All RCs were classified into one of the following 13 NPS types: synthetic cathinone, synthetic cannabinoid, benzodiazepine, opioid, arylcyclohexylamine, tryptamine, LSD analogs, 2C, aminoindane, benzofuran, phenidate derivative, amphetamine, and other.

Results: During the study period, two sites became non-functional but were included in the final analysis. The mean number of RCs per site was 52 (range 10-141) for a total of 631 RCs. There was at least one example of all 13 NPS types identified. The two most common types of RC listed were synthetic cathinones and synthetic cannabinoids with a mean of 16 (range 0-39) and 14 (range 0-33) per site, respectively. The least common type of RC encountered was LSD analogs with total of four documented. A total of 68 new RCs were added during the 12 weeks. Synthetic cathinones were the most common newly listed RC $(n=17)$. A total of 20 RCs disappeared. Synthetic cannabinoids were the most common $\mathrm{RC}$ to disappear $(n=8)$.

Discussion: Distribution of NPS as RCs via the internet is a known challenge. This study demonstrates that a large inventory of RCs can be found online with fluctuations in inventory consistent with the ever evolving nature of NPS use. A limitation of this study is that we could not verify the veracity of the labeled substances.

Conclusion: This study demonstrates that RCIVs advertise a large and dynamic number of NPS.

\section{Incidence and Risk Factors for Hyperlactatemia in ED Patients} with Acute Metformin Overdose

Emily Taub $^{1}$, Robert Hoffman ${ }^{2}$, Alex Manini ${ }^{1}$.

${ }^{T}$ Icahn School of Medicine at Mount Sinai, New York, NY, USA

${ }^{2}$ NYU School of Medicine, New York, NY, USA

Background: Metformin causes hyperlactatemia by inhibiting hepatic lactate uptake and the conversion of lactate to glucose. Lactic acidosis is a known complication, but clinical risk and prognosis remain unclear.

Research Question: To describe the incidence of hyperlactatemia and clinical risk factors for lactic acidosis in patients with acute metformin overdose. Methods: This was a secondary data analysis of a prospective observational cohort of adult ED patients presenting with acute drug overdose at two urban tertiary care hospitals over 5 years. Chronic, pediatric, and nondrug overdoses were excluded as were those missing outcome information. We collected demographics, exposure details, laboratory information, initial serum lactate, and extracorporeal indications per EXTRIP guidelines. Missing lactate data were accounted for by multiple imputations using a derived bicarbonate correlation. The outcomes were hyperlactatemia (lactate $\geq 2 \mathrm{mmol} / \mathrm{L}$ ) and lactic acidosis (MALA, lactate $\geq 5 \mathrm{mmol} / \mathrm{L}$ with $\mathrm{pH}<7.35$ ). Assuming $20 \%$ outcome prevalence, we needed 50 patients to show twofold increased risk with $80 \%$ power and $5 \%$ alpha. Clinical risk factors for MALA were derived using multivariable logistic regression in SPSSv22.

Results: We screened 3739 acute overdoses; 2872 met eligibility, and 56 self-reported metformin overdose (57\% female, mean age $55.7,0 \%$ endstage renal disease). There was a high incidence of hyperlactatemia during hospital stay (53.6\%); MALA was less frequent (30.4\%); there were no inpatient deaths. Initial serum bicarbonate and lactate were highly correlated $\left(r^{2}=0.63, p<0.01\right)$. Repeat serum lactate increased in only three patients (hyperlactatemia rose by $1.8 \%$, MALA by $3.8 \%$ ). EXTRIP guidelines indicated hemodialysis for three, all of whom received it. Clinical risk factors for MALA were lower $\mathrm{PCO}_{2}(p=0.02)$, older age ( $5 \%$ increased risk per year of age, $p=0.078$ ), and acetaminophen coexposure (adjusted $\mathrm{OR}=15.6, p=0.07$ ). 
Discussion: These data suggest a good prognosis for ED patients with acute metformin overdose; hyperlactatemia occurred in over half, but MALA in less than one-third. Additionally, indications for hemodialysis were rare, and none died. There was minimal utility in trending lactate, as rising lactate was exceedingly rare.

Conclusion: Hyperlactatemia was common in ED patients with acute metformin overdose. Independent clinical risk factors for MALA were acetaminophen co-exposure, compensatory respiratory alkalosis, and older age.

\section{Thistle While You Work: A Case of Mushroom Poisoning with Liver Recovery Evident Before Silibinin Administration}

Ryan Surmaitis ${ }^{1,3}$, Margaret Pietrowski ${ }^{2}$, Paul Kolecki ${ }^{2,3}$, Jane Miloradovich $^{3}$, David Vearrier ${ }^{1,3}$, Kevin Osterhoudt ${ }^{3}$.

${ }^{1}$ Drexel University College of Medicine, Philadelphia, PA, USA

${ }^{2}$ Thomas Jefferson University Hospital, Philadelphia, PA, USA

${ }^{3}$ The Poison Control Center at The Children's Hospital of Philadelphia, Philadelphia, PA, USA

Background: Ingesting some species of Amanita mushrooms can lead to hepatic failure and death. Although many therapies have been speculated to be potentially beneficial, there is no uniformly accepted standard treatment. We present a case of suspected amatoxin-induced hepatic failure initially treated with hydration, $\mathrm{N}$-acetylcysteine (NAC), and multi-dose activated charcoal (MDAC). The patient was later enrolled in a clinical trial involving silibinin. The time course of hepatic recovery in relation to silibinin administration is compared, and relevant research implications are considered.

Case Report: A 49-year-old man, with history of untreated hepatitis B, presented with vomiting and diarrhea $12 \mathrm{~h}$ after ingestion of foraged mushrooms. Physical exam and laboratory studies were unremarkable. He was hospitalized for hydration and serial hepatic monitoring. Within $24 \mathrm{~h}$, the patient's serum hepatic transaminases rose to AST $289 \mathrm{U} / \mathrm{L}$ and ALT $286 \mathrm{U} / \mathrm{L}$, and IV NAC and enteral MDAC were administered. The man became jaundiced and hepatic deterioration was evident with lab values at 72 h post-ingestion: AST $6962 \mathrm{U} / \mathrm{L}$, ALT $6777 \mathrm{U} / \mathrm{L}$, and INR 6.7. Contact was made to enroll the patient in a national open-label trial of silibinin which was procured after an additional $20 \mathrm{~h}$. Liver improvement was evident before initiation of silibinin: AST 1477 U/L, ALT 4017 U/L, and INR 2.5. Silibinin was continued for a 5-day protocol; the man was discharged on hospital day 9 with normal serum biomarkers of liver function.

Discussion: Silibinin is an extract from milk thistle thought to impair hepatocellular uptake of amatoxin. It is available in the U.S. for antidotal administration via clinical trial. Hepatic recovery was evident in our patient, in a sensible time course, before silibinin administration. The analysis and interpretation of this non-randomized open-label clinical trial must be performed carefully, as the association of recovery to use of silibinin is this patient is unlikely to be causal. Indeed, had silibinin been procured sooner and used earlier in the hospital course, this case may have falsely appeared to be a silibinin-associated "save."

\section{Cocaine and a Broken Heart (Syndrome)}

Matthew Stripp, William Kerns

Carolinas Medical Center, Charlotte, NC, USA

Background: Takotsubo cardiomyopathy or "broken heart syndrome" is a condition characterized by left ventricular apical ballooning and decreased contractility. It is an unusual consequence of high-catecholamine state and known triggers include amphetamine intoxication. We present a case of cocaine-associated chest pain with Takotsubo physiology.

Case Report: A 53-year-old female with past medical history of depression, anxiety, and nicotine use sought care for severe, progressive lower chest pain, nausea, and vomiting that began 2 weeks prior. The patient attributed the discomfort to a punch to the chest sustained during an altercation. Aside from marijuana, she denied any other recreational substances. She was agitated, afebrile, bradycardic (55 bpm), and mildly hypertensive $(154 / 76 \mathrm{mmHg})$. ECG demonstrated sinus bradycardia and nonspecific ST wave abnormalities; unchanged from a prior EKG. Chest radiograph was normal. Troponin I was elevated: $1.68 \mathrm{ng} / \mathrm{ml}$ (ref 0.00-0.07) Renal indices, hemoglobin, liver function, lipids, and lipase were unremarkable.

The patient was admitted for suspected non-ST elevation infarction. Immunoassay urine drug screen was positive for cocaine metabolite and negative for amphetamine. Echocardiography revealed an ejection fraction of $30-35 \%$, a small circumferential pericardial effusion, and severe hypokinesis of the left ventricle from mid to apex with sparing of the base consistent with Takotsubo physiology. Echocardiogram 2 years prior was normal. CT angiography of the chest and coronary arteries was normal. Serial troponin I peaked at $3.72 \mathrm{ng} / \mathrm{ml}$. She was discharged home on day 3 with mild residual chest pain.

At 1-month follow-up, she complained of mild persistent chest pain and dyspnea. She reported cocaine and nicotine cessation (UDS negative for cocaine), but failed to follow up for echocardiogram.

Case Discussion: This patient's clinical scenario and diagnostic studies were consistent with cocaine-induced Takotsubo syndrome. Other etiologies for chest pain were excluded. While cocaine is responsible for a variety of commonly recognized cardiovascular pathology including ischemia, cardiomyopathy, dysrhythmias, and cardiac arrest, it is not typically considered in cases of Takotsubo and is rarely reported in the literature.

Conclusion: Consider Takotsubo cardiomyopathy in the differential diagnosis for patients presenting with cocaine-associated chest pain. Echocardiography is recommended for diagnostic screening.

\section{Acephate: A Safer Pesticide Alternative?}

Matthew Stripp ${ }^{1,2}$, Michael Beuhler ${ }^{2,1}$.

${ }^{T}$ Carolinas Medical Center, Charlotte, NC, USA

${ }^{2}$ Carolinas Poison Center, Charlotte, NC, USA

Background: Acephate is a weak organophosphate (OP) that is hydrolyzed in insects to methamidophos (MP), a potent OP. Rats metabolize acephate primarily to des-O-methylacephate as MP is thought to inhibit its own formation in mammals. This is the basis for the presumed selectivity for insects. The effects of AP in humans are poorly characterized and the role of treatment with antimuscarinic and oxime therapy is unknown. We present a case series of three patients with acute ingestion of acephate.

Case Reports: Patient 1 is a 32-year-old female who presented with erratic behavior, diaphoresis, vomiting, and rapid heart rate after ingesting acephate mixed in water following an altercation. She had "hypoactive delirium," confusion, diarrhea, sialorrhea, tachypnea (33 bpm), hypothermia $\left(94.5^{\circ} \mathrm{F}\right)$, and tachycardia $(117 \mathrm{bpm})$. Lung exam was normal. The patient was started on pralidoxime. She developed respiratory secretions requiring atropine, acidosis treated with bicarbonate infusion, and hypotension refractory to IV hydration requiring norepinephrine infusion. Resolution of cholinergic symptoms was noted $96 \mathrm{~h}$ from ingestion.

Patient 2 is a 16-year-old female who presents after ingesting "a spoonful" of acephate with suicide intent. She had pallor, diaphoresis, pinpoint pupils, tachycardia, emesis, diarrhea, abdominal pain, and confusion. The patient later had generalized weakness, flat affect, and poor muscle tone. She was treated with pralidoxime and medically cleared at $70 \mathrm{~h}$ post ingestion. Pseudocholinesterase was 2.5 (ref 4.9-11.9).

Patient 3 is 50 -year-old female who presented with vomiting, dilated pupils, tachycardia, lethargy, and acidosis after reported ingestion of boric acid and acephate mixed with water. The patient did not develop bronchorrhea; however, she received atropine for nausea $52 \mathrm{~min}$ after ED arrival. She was treated with pralidoxime. Symptoms resolved by $23 \mathrm{~h}$ post ingestion. 
All three patients became symptomatic less than $5 \mathrm{~h}$ after reported ingestion.

Case Discussion: Acephate exposures are poorly characterized and previously thought to be unlikely to produce significant toxicity due to differing metabolism between insects and mammals. The three patients with varied presentations all exhibited cholinergic symptoms. However, some of patient 3's symptoms could be attributed to boric acid ingestion.

Conclusion: Acephate can cause significant and varied presentations of cholinergic toxicity in humans.

\section{Occult Occupational Ocular Exposure}

Matthew Stripp $^{1,2}$, Anna Dulaney ${ }^{2,1}$, Michael Beuhler ${ }^{2,1}$, Ricky Langley ${ }^{3}$ ${ }^{T}$ Carolinas Medical Center, Charlotte, NC, USA

${ }^{2}$ Carolinas Poison Center, Charlotte, NC, USA

${ }^{3}$ North Carolina Division of Public Health, Raleigh, NC, USA

Background: Ultraviolet (UV) light is electromagnetic radiation shorter in wavelength than visible light but longer than X-rays. UV radiation is subdivided into bands: UV-A (315-400 nm), UV-B (280-315 nm), and UV-C (100-280 nm). Sunlight at the Earth's surface is primarily UV-A and UV-B after attenuation by Earth's atmosphere. UV-C spectrum radiation is generated by arc welding, improperly shielded metal halide lights, and germicidal UV applications.

Case Report: Eighteen of 85 restaurant employees complained of ocular burning, irritation, dry eyes, pruritus, and an associated facial rash. The symptoms affected employees working over a 2-day period. Managers, wait staff, and kitchen staff all reported symptoms. The face and upper body were primarily affected to varying degrees. There were no respiratory complaints. Two employees visited a local emergency department and one was diagnosed with "chemical burn/irritation of cornea." The poison center, the local health department, appliance/supply and service vendors, and the pesticide company were contacted along with other state agencies.

Investigation of the metal halide light bulbs present in the restaurant's fly light traps by a licensed electrical contractor did not reveal any damage and were reportedly in good working order. The following day, that electrician noticed skin burns around the area of his UV protective eyewear. Upon further inspection, UVC spectrum germicidal light bulbs instead of standard UV replacement bulbs had been inadvertently shipped by the supplier and installed in the fly light traps.

Case Discussion: UV radiation-induced photokeratitis is an injury with a delayed presentation with symptoms beginning 2-12 h after exposure. The presentation can mimic widespread toxin exposure. Affected individuals are expected to make a full recovery from acute symptoms. However, increased lifetime carcinogenic risk is difficult to quantify. Conclusion: UVC radiation injury should be considered in the differential for groups of patients with simultaneous development of dermal and eye symptoms. Exposure to UVC radiation may not always be apparent and careful history taking and a coordinated effort may be required to identify the etiology in a timely fashion.

\section{Prolonged \& Severe Opioid Toxicity after Ingestion of Poppy Seed Tea}

Meghan Spyres ${ }^{1}$, Xander van Wijk ${ }^{2}$

${ }^{T}$ University of Southern California, Los Angeles, CA, USA

${ }^{2}$ University of California, San Francisco Department of Laboratory Medicine, San Francisco, CA, USA

Background: The severity and penetrance of the opioid epidemic has resulted in both widespread physician concern and increased public awareness. Opioid substitutes are discussed with increased attention. The public's perceived safety of such opioid alternatives, however, may be misleading, and the scientific literature is lacking. This is a case report of a significant opiate overdose after consumption of poppy seeds purchased on the internet.
Methods: A 33-year-old man consumed a homemade poppy seed tea for symptoms of chronic anxiety. The tea was prepared mixing $2 \mathrm{lbs}$ of poppy seeds with water and shaking vigorously for $10-15 \mathrm{~min}$. Soon after ingestion of one half the mixture, the patient felt intoxicated. Aside from his daily medications of alprazolam, escitalopram, and buspirone taken $12 \mathrm{~h}$ prior, no other substances were ingested. The patient was found to be unarousable by his wife 10-12 h after ingestion. EMS was called and naloxone was administered with complete reversal of sedation. He was transferred to the ED, observed for 5-6 h, and discharged home after receiving $1 \mathrm{mg}$ alprazolam. The patient reported a persistent feeling of intoxication during his ED stay prior to alprazolam administration. One hour after arrival home, he was found unarousable and cyanotic. EMS was called and naloxone was administered again with reversal of symptoms. In the ED, the patient had a normal mental status and normal vital signs. Urine drug screen was positive for opiates and benzodiazepines. He was admitted to the ICU for observation. No further naloxone administration was required. The tea was tested with LC-MS/MS and was positive for morphine $(1.09 \mathrm{mg} / \mathrm{mL})$ and codeine $(62.1 \mathrm{mcg} / \mathrm{mL})$ only. No additional opioids, including fentanyl, methadone, hydrocodone, and oxycodone, were found. Discussion: Poppy seeds are known to contain opiate alkaloids including morphine and codeine. Although detectable serum morphine levels after poppy seed ingestion have been reported, a clinically significant opioid toxidrome has not been described. Given the current opioid epidemic, physicians should be aware of the public's use of alternative agents that may result in significant toxicity.

Conclusion: Poppy seed ingestion may result in significant opiate toxicity requiring naloxone reversal.

\section{Toxicologic Mimic: Argyria-like Skin Discoloration Associated with Methemoglobinemia}

Joshua Shulman $^{1,2}$, Andrew Farkas ${ }^{1,2}$, Joseph Yanta ${ }^{1,2}$

${ }^{T}$ UPMC Division of Medical Toxicology, Pittsburgh, PA, USA

${ }^{2}$ UPMC Department of Emergency Medicine, Pittsburgh, PA, USA

Background: Alkaptonuria is a rare autosomal recessive disorder of tyrosine and phenylalanine metabolism. It leads to overproduction of homogentisic acid (HGA), a tissue pigment and inducer of methemoglobin. It leads to ochronosis, a blue-gray tissue discoloration that can mimic silver deposition (argyria), usually starting after the fourth decade of life. Hypothesis: In the setting of end-stage renal disease, alkaptonuria can cause skin pigmentation mimicking argyria and severe methemoglobinemia.

Methods: This is a retrospective single chart review. Hospital and autopsy records were reviewed.

Case Report: A 60-year-old woman with a history of worsening slate blue-gray skin discoloration, renal failure on hemodialysis, and pseudobulbar affect, on quinidine/dextromethorphan, presented to a community hospital for evaluation of weakness, where she was found to have a methemoglobin level of $10 \%$ and hemoglobin of $6.0 \mathrm{~g} / \mathrm{dL}$. After transfer to tertiary center, her repeat methemoglobin level was $24.5 \%$ with hemoglobin of $8.1 \mathrm{~g} / \mathrm{dL}$. She was given $2 \mathrm{mg} / \mathrm{kg}$ of methylene blue and transfused 2 units packed red blood cells. Subsequently, she became hypotensive. She underwent an 8-unit manual exchange transfusion, in addition to, pressors, continuous renal replacement, and broad-spectrum antibiotics. Care was withdrawn after the patient required intubation for acute respiratory distress syndrome; she died on hospital day 2. Post-mortem analysis showed findings consistent with alkaptonuria. Pre-mortem serum silver testing was negative. Urine gas chromatography/mass spectrometry did not identify other methemoglobin-inducing substances. Confirmatory genetic testing was not available.

Discussion: Alkaptonuria occurs due to an inborn error of tyrosine and phenylalanine metabolism due to dysfunction of homogentisate 1,2dioxygenase. It leads to deposition of HGA products in the skin, causing blue-gray discoloration that presents later in life. HGA is a known inducer of methemoglobin production. It is unclear what role quinidine played in the development of methemoglobinemia or ochronosis. 
Conclusion: Development of methemoglobinemia in the setting of bluegray skin discoloration should prompt consideration of alkaptonuria as an etiology.

\section{Don't Sweat the Small Stuff: Galantamine Toxicity in Polysubstance Overdose}

Shirley Shao ${ }^{2}$, Joshua Shulman ${ }^{1,2}$, Michael Lynch ${ }^{1,2}$

${ }^{T}$ UPMC Division of Medical Toxicology, Pittsburgh, PA, USA

${ }^{2} U P M C$ Department of Emergency Medicine, Pittsburgh, PA, USA

Background: Galantamine is an alkaloid substance used in the treatment of moderate to severe Alzheimer's disease. It is a reversible and competitive acetylcholinesterase inhibitor that acts centrally as well as peripherally. Reports of toxicity are rare.

Hypothesis: In a complicated polysubstance overdose, galantamine can cause significant cholinergic toxicity.

Methods: This is a retrospective single case report utilizing both prehospital and hospital records. A previously healthy 23 -year-old man was found unresponsive in his bathtub. He was a known heroin user. $\mathrm{He}$ was given $4 \mathrm{mg}$ naloxone intravenously, causing agitation, and was intubated for airway protection. On clinical examination, the patient was found to be in sinus tachycardia with a heart rate of 120 beats per minute and a temperature of $38.5^{\circ} \mathrm{C}$. He was profoundly diaphoretic with copious airway secretions necessitating frequent suctioning. He had miosis on clinical examination.

Results: Urine gas chromatography/mass spectrometry revealed galantamine, in addition to cocaine metabolites, fentanyl, codeine, oxycodone, quinine, and diphenhydramine. His course was complicated by compartment syndrome necessitating fasciotomy and renal failure requiring hemodialysis. After a prolonged intensive care stay, he was discharged home with home health care.

Discussion: Our patient had no preexisting medical conditions that would have accounted for the galantamine that was found. On its own, galantamine toxicity would be expected to cause excess muscarinic agonism due to increased synaptic acetylcholine. His presentation was complicated by the presence of cocaine and possible naloxone-induced opioid withdrawal. However, the dramatic demonstration of respiratory secretions and diaphoresis suggest a cholinergic toxidrome. Sinus tachycardia could have been due to catecholamine activity from cocaine and/or opioid withdrawal; however, preganglionic sympathetic stimulation by acetylcholinesterase inhibition, overcoming postganglionic parasympathetic effects, may have also contributed to or accounted for tachycardia. It is unclear whether galantamine was a coingestant or an adulterant in this case. This is the first reported case of galantamine toxicity coinciding with opioid overdose.

Conclusion: The patient's clinical presentation was multifactorial; however, given the degree of diaphoresis and respiratory secretion, cholinergic toxicity was a likely contributing factor.

\section{Intestinal Botulism: The Lurking Threat of Bariatric Surgery}

Lindsay Schaack ${ }^{2}$, Jessica Weiland ${ }^{1,2}$, Alaina Steck ${ }^{1,2}$

${ }^{7}$ Emory University School of Medicine, Atlanta, GA, USA

${ }^{2}$ Georgia Poison Center, Atlanta, GA, USA

Background: Bariatric surgery offers an effective means to combat obesity. However, manipulation of the gastric anatomy may increase one's risk for contracting intestinal botulism.

Hypothesis: Gastric bypass surgery increases the risk of intestinal botulism

Case Report: This is a single patient case report of a 33-year-old female with a past medical history significant for gastric bypass surgery presented to the emergency department complaining of lower extremity weakness resulting in an inability to ambulate. Within hours of her arrival, she developed shortness of breath, blurry vision, slurred speech, and symmetric flaccid paralysis which rapidly progressed to respiratory failure requiring intubation.
An extensive work-up failed to reveal the cause of her paralysis. On hospital day 10 , she was transferred to a tertiary care facility which, suspecting botulism, administered antitoxin. She began to show improvement on day 16 , demonstrating voluntary horizontal eye movement. On day 17 , her test results came back positive for botulinum toxin type $F$. The patient had gradual recovery of motor function over the next several weeks and was successfully weaned from the ventilator on day 28 . She was transferred to inpatient rehab on day 37 and discharged home on day 53 with no neurologic deficits.

Discussion: The obesity epidemic represents a growing burden on the nation's health and cost of healthcare. Bariatric surgery offers an effective treatment for severe obesity; however, manipulation of the gastric anatomy through bypass procedures alters the microbiota of the intestine, frequently leading to colonization. Typically, cases of intestinal toxemia botulism require anatomic or physiologic disruption of gastrointestinal flora, such as gastrointestinal surgery or antimicrobial treatment. Case reports of botulism type F implicate pyloroplasty, Crohn's disease, and gastric stapling as potential risk factors. To our knowledge, this is the first case report of intestinal toxemia botulism occurring in an otherwise healthy adult with a history of gastric bypass.

Conclusion: Gastric bypass surgery may increase one's risk of contracting intestinal botulism via alteration of gut flora, similarly to previously identified risk factors. As these procedures become more commonplace, more cases may emerge due to the ubiquitous nature of the bacteria and inherent difficulty in its avoidance.

\section{A Case Series of Ocular Exposure to Euphorbia Plant Species}

\section{Ryan Sarkaria, Jeff Lapoint}

Department of Emergency Medicine, Kaiser Permanente Souther California, San Diego, CA, USA

Background: Euphorbia is a diverse genus of plants with a worldwide distribution that are frequently used in ornamental landscaping. These plants produce a milky, white sap which contains an irritating latex. Ocular exposure to the sap leads to severe eye pain and injury ranging from keratoconjunctivitis to possible blindness.

Purpose: The purpose of this study is to report the course of ocular exposure to Euphorbia plants in patients with early presentation for emergency evaluation who received supportive care and outpatient follow-up. Methods: This is a case series of ocular exposures to Euphorbia plant species. A retrospective review of patient charts was performed to obtain study data.

Results: A total of 34 cases were documented. All cases were early presenters within the first $24 \mathrm{~h}$ of exposure. Findings on initial ocular examination included conjunctival injection, keratitis, corneal abrasion, chemosis, and/or blepharitis. 19/34 (55.9\%) had intraocular pressure and $30 / 34(88.2 \%)$ had ocular $\mathrm{pH}$ documented on initial examination. 26/34 (76.5\%) had visual acuity documented on initial presentation. 17/34 (50\%) had visual acuity documented on follow-up presentation. Treatment consisted of irrigation 34/34 (100\%), topical anesthetic drops $11 / 34$ (32.4\%), and/or oral pain control 2/34 (5.9\%). All 34 (100\%) cases were discharged home after evaluation and treatment. Home prescriptions included antibiotic drops 29/34 (85.3\%), lubricant eye drops $20 / 34$ $(58.8 \%)$, and oral or topical pain medications 13/34 (38.2\%). 31/34 $(91.2 \%)$ had documented outpatient follow-up, none of which had significant residual visual deficits or apparent long-term sequelae.

Discussion: Exposure to the sap of Euphorbia species in this series lead to pain and localized injury. Intraocular pressure and $\mathrm{pH}$ were not significantly affected and were not predictors of recovery or severity of injury. Antibiotic drops were prescribed in the majority of cases; however, their necessity cannot be determined from this series. Ophthalmologic followup did not affect outcome. No acute ocular emergency or permanent ocular damage was documented on initial presentation or follow-up.

Conclusion: Early evaluation and supportive care is the appropriate treatment. Ocular Euphorbia exposure is not uncommon and emergency providers should be aware of the need for early supportive care. 
66. The Global Education Toxicology toolKIT (GETKIT): A One Day Course for Teaching Medical Toxicology in Developing Countries

Cynthia Santos $^{1,2}$, Anselm Wong ${ }^{3}$, Rais Vohra ${ }^{4}$, Kathryn Kopec ${ }^{5}$, Ziad Kazzi ${ }^{1,2}$

${ }^{1}$ Emory University Hospital, Atlanta, GA, USA

${ }^{2}$ Centers for Disease Control, Atlanta, GA, USA

${ }^{3}$ Austin Health, Monash University, University of Melbourne, Melbourne, Australia

${ }^{4}$ University of California San Francisco Fresno, Fresno, CA, USA

${ }^{5}$ Carolinas Medical Center, Charlotte, NC, USA

Background: Poisoning is a global public health problem, yet there are limited educational opportunities for healthcare professionals in developing countries to learn medical toxicology.

Hypothesis: Attendance at an introductory medical toxicology course for emergency health care providers in the Dominican Republic (DR) and India will improve attendees' medical toxicology knowledge base.

Methods: Faculty and fellows from the American College of Medical Toxicology administered a one day introductory course in the DR (May 2016) and India (June 2016). Lecture topics included initial management, toxidromes, interpretation of toxicology laboratory testing results, pesticides, pediatric toxicology, hazardous materials, and envenomations. Lectures were administered in either Spanish (DR) or English (India). Two different sets of sixteen pre- and post-course multiple choice questions were used to evaluate baseline knowledge and course content retention, respectively. Qualitative feedback was elicited using pre- and post-course tests. Participants were also asked about previous toxicology experience and course satisfaction in the post-test. We compared pre- and post-test scores using a paired $t$ test in SAS 9.3.

Results: There were a total of 41 course participants ( $n=7$, DR; $n=34$, India) who attended the GETKIT course. Participants' training levels ranged from the first to third year emergency medicine residents in three large, urban metropolitan centers (Cabral Hospital in Santiago, DR; Kokilaben Ambani Hospital I Mumbai, India; and Madurai Meenakshi Mission Hospital and Research Centre, Madurai, India). All testing and feedback were anonymous. Mean pre-test scores were similar in both countries (53\% DR, 54\% India). Mean post-test scores were $72 \%$ in both countries. The paired $t$ test indicated a significant difference between preand post-test scores in each country individually and in both countries combined. Most participants $(n=36,88 \%)$ indicated that toxicology education was lacking in their medical center, and all participants would recommend the course to their colleagues.

Discussion: The improvement before and after the course is a positive indicator that the course was effective and the results were consistent across country, language, and instructor changes.

Conclusion: A one day, interactive medical toxicology curriculum to health care providers in countries without toxicology services can be used to deliver essential toxicology education and significantly improve participant knowledge.

67. Characterizing Chemical Terrorism Incidents Collected by the Global Terrorism Database, 1970-2014

Cynthia Santos $^{1,2}$, Mehruba Anwar ${ }^{1,2}$, Jessica Weiland ${ }^{1,2}$, Joshua Schier $^{1,2}$

${ }^{1}$ Emory University Hospital, Atlanta, GA, USA

${ }^{2}$ Centers for Disease Control and Prevention, Atlanta, GA, USA

Background: The Global Terrorism Database (GTD) is an open-source database on terrorist incidents around the world since 1970 and is maintained by the National Consortium for the Study of Terrorism and Responses to Terrorism, a Department of Homeland Security Center of Excellence.

Objective: We characterized chemical terrorism incidents reported to the GTD from 1970 through 2014 to address the following: What kinds of chemical terrorism agents were used among the incidents reported in the GTD? Where did they occur? How much morbidity and mortality were associated with these events?

Methods: We included incidents that were categorized as "chemical" when searching the GTD database and excluded all others. We reviewed each incident to create subtypes based on agent similarities: corrosives, chemical explosives, cyanide, metals, nerve gas, pesticides, tear gas, gas (unspecified), and unknown. We analyzed the total number of chemical terrorism incidents between 1970 and 2014 and calculated the mean number of injuries, fatalities, and frequencies by region and agent subtype.

Results: Of the 156,745 terrorism event reports during 1970-2014, chemical terrorism incidents consisted of $<0.2 \%(n=267)$. The most commonly reported chemical terrorism subtypes were as follows: unknown $(n=81,30.3 \%)$; corrosives $(n=65,24.3 \%)$; tear gas $/$ mace $(n=31,11.6 \%)$; unknown gas $(n=26,9.7 \%)$; cyanide $(n=24,9.0 \%)$; pesticides $(n=15,5.6 \%)$; metals $(n=19,7.1 \%)$; and nerve gas $(n=6$, $2.2 \%$ ). On average, 53 injuries (range 2.5-1622) and 8 deaths (range 0.0 $224.3)$ resulted per chemical terrorism incident. Nerve gases $(n=6)$ had the highest reported mean number of fatalities (224) and mean number of injuries (1622) per incident. Tear gas was the most commonly reported agent in South America $(n=8,29.6 \%)$ and North America $(n=3,20.0 \%)$ while corrosives were the most common in the Middle East $(n=14$, 48.3\%) and South Asia $(n=19,27.5 \%)$.

Discussion: In this dataset, tear gas and corrosives were the chemical agents implicated most often while nerve agents were the most lethal. However, more than a third of the agents were unknown, suggesting a lack of reliable data. The wide variety of chemical agents used suggests that a broad education for preparedness may be needed.

Conclusions: Our data suggest that morbidity and mortality vary by chemical subtype and by region. Results may be helpful in developing regional chemical terrorism preparedness activities.

\section{Infusion of $70 \%$ Dextrose to Minimize Risk of Fluid Overload in High Dose Insulin Therapy: A Case Series}

Haley Robinson ${ }^{1}$, Kelly Considine ${ }^{1}$, Jon Cole ${ }^{1,2}$

${ }^{1}$ Hennepin County Medical Center, Minneapolis, MN, USA

${ }^{2}$ Minnesota Poison Control System, Minneapolis, MN, USA

Background: Patients receiving high dose insulin (HDI) are often on multiple infusions and at high risk for pulmonary edema. Concentrating infusions minimizes this risk. A 70\% stock solution of dextrose (D70) is often used to compound dextrose solutions; however, reports of direct infusion of D70 are lacking.

Hypothesis: Infusion of D70 as part of HDI is safe and feasible, and may reduce the total volume of fluid infused.

Methods: This is a two-patient case series.

Results: A 25-year-old woman presented in respiratory failure secondary to iatrogenic opioid and sedative overdose and aspiration from elective procedural sedation. She received 41 of crystalloid and IV metoprolol for physiologic tachycardia. On emergency department arrival, she was hypotensive $(74 / 41 \mathrm{mmHg})$ and hypoxic $(83 \%)$ with a pulse of 83 beats/ min. Bedside echo revealed poor contractility and pulmonary edema; thus, HDI was initiated given the proximal beta-blocker with relative bradycardia in the setting of hypotension. She stabilized after an HDI bolus of 59 units; D70 was infused for $3 \mathrm{~h}$. The patient was discharged on day 3 without sequelae.

A 44-year-old woman presented to a rural hospital comatose with a suspected overdose of carbamazepine and atenolol. She was intubated and started on the following infusions: HDI 31 units $/ \mathrm{h}(0.2 \mathrm{units} / \mathrm{kg} / \mathrm{h})$, dopamine $20 \mathrm{mcg} / \mathrm{kg} / \mathrm{min}$, and norepinephrine $0.2 \mathrm{mcg} / \mathrm{kg} / \mathrm{min}$. Upon tertiary center arrival, she was in pulmonary edema with the following vital signs: $67 / 45 \mathrm{mmHg}, 93$ beats $/ \mathrm{min}$, and $\mathrm{SpO}_{2} 84 \%$. HDI was titrated to 8.5 units $/ \mathrm{kg} / \mathrm{h}$. D70 was infused for $110 \mathrm{~h}$. She was discharged with a tracheostomy secondary to ARDS. At discharge, she was alert and following commands. 
Discussion: D70 has an osmolarity of $3535 \mathrm{mOsm} / \mathrm{L}$. While high osmolarity solutions may cause phlebitis and thrombosis, risk is minimized if central venous access is used. The osmolarity of D70 is dwarfed by other medications used in critical care; for example, $23.4 \%$ saline has an osmolarity of $8008 \mathrm{mOsm} / \mathrm{L}$. D70 offers a significant concentration advantage. For example, patient 2 received 4.21 of dextrose-containing fluid; if D10 had been used, she would have received 30 additional liters.

Conclusion: Infusion of D70 as part of HDI appears safe and feasible.

\section{Acute Hepatitis Following Self Administration of Fenbendazole,} A Veterinary Antihelmintic

Angela Regina ${ }^{1}$, Payal Sud ${ }^{2}$, Joshua Nogar ${ }^{3}$

${ }^{T}$ St Barnabas Health System, Bronx, NY, USA

${ }^{2}$ Long Island Jewish Hospital, New Hyde Park, NY, USA

${ }^{3}$ North Shore University Hospital, Manhasset, NY, USA

Introduction: Patients frequently seek non-FDA-approved treatments for controversial diagnoses. We describe a case of toxicity from internet-procured fenbendazole in a patient self-treating "chronic Lyme disease."

Case: A 46-year-old woman presented to the emergency department (ED) with jaundice and pruritus. She had a history of depression, but denied using any prescription medications. In the ED, she admitted to using a "goat dewormer," fenbendazole, which she purchased on the internet in order to treat her chronic Lyme disease and other internal parasites. Two weeks prior, she switched to a formulation with ten times the potency. She also admitted to taking other supplements but had not taken them in over 2 months. In the ED, she had unremarkable vital signs, and her physical exam was significant for jaundice, scleral icterus, and extensive skin excoriations. Laboratory analysis was revealed a total bilirubin of $5.7 \mathrm{mg} / \mathrm{dL}$, direct bilirubin on $4.3 \mathrm{mg} / \mathrm{dL}$, and AST and ALT of 529 and $470 \mathrm{U} / \mathrm{L}$, respectively. She was treated with antihistamines and advised to stop all medications. She underwent an extensive work-up, including testing for other causes of her hepatic dysfunction, which were negative. She improved and was discharged 2 days later. A liver biopsy was performed and findings were consistent with drug-induced liver injury.

Discussion: Chronic Lyme disease is a diagnosis used for patients with pain and neurocognitive symptoms with or without evidence of previous Lyme disease [1]. The diagnosis is not widely accepted in the medical community and patients often turn to the Internet to self-treatment modalities. To the best of our knowledge, there has not been a documented case of acute hepatitis following administration of fenbendazole. Fenbendazole is a member of the benzimidazole class of antihelmintics. Other medications within this class include mebendazole and albendazole. Cases of human hepatotoxicity have been reported within this class of medication, and due to their structural similarities, fenbendazole is likely hepatotoxic as well $[2,3]$. Unfortunately, serum levels of the drug were unable to be obtained.

Conclusion: Physicians should be aware of the possibility of patients purchasing veterinary medications on the internet and their adverse reactions and toxicities.

\section{Unintentional Levetiracetam Overdose in an Eleven-Week-Old Infant Without Delayed Effects on One-Year Follow Up}

Angela Regina ${ }^{1}$, Benjamin Kessler $^{2}$, Nima Majlesi ${ }^{2}$

${ }^{T}$ St Barnabas Hospital, Bronx, NY, USA

${ }^{2}$ Staten Island University Hospital, Staten Island, NY, USA

Introduction: Levetiracetam is a commonly used and well-tolerated anticonvulsant. The most common adverse events are somnolence, irritability, asthenia, and dizziness. ${ }^{1}$ Serious systemic effects are rarely seen. ${ }^{2}$

We present the case of a large pediatric overdose with long-term follow-up.
Case: An 11-week-old 3.6-kg male with no medical history was unintentionally given $170 \mathrm{mg}$ of liquid levetiracetam $(47 \mathrm{mg} / \mathrm{kg})$ instead of the child's famotidine. In the emergency department (ED), he had normal vital signs, was acting at his baseline, and had a normal neurologic examination. He was admitted overnight for observation and was discharged home the following day. His levetiracetam concentration was $55.4 \mathrm{mcg} / \mathrm{mL}$. Telephone follow-up 1 year later revealed him reaching all appropriate milestones with no focal deficits and no developmental delay.

Discussion: The mechanism of action of levetiracetam is incompletely understood and it undergoes minimal metabolism. ${ }^{2}$ With good renal function, the elimination half-life was found to be similar in overdose and therapeutic use. ${ }^{3}$ The maximum recommended dose of levetiracetam in children less than 6 months of age is $42 \mathrm{mg} / \mathrm{kg}$ divided twice daily.

The patient did not have a seizure history and received one large dose of levetiracetam $47 \mathrm{mg} / \mathrm{kg}$. We believe this to be the youngest case of a confirmed levetiracetam overdose. The child did not experience any compilations and 1 year later showed no long-term effects.

Previous literature reported the youngest case of overdose in a 10-monthold child with seizure history. The child received a tenfold overdose over 35 days and presented to the hospital somnolent and returned to baseline after 2 days of supportive care and observation; no levetiracetam concentrations were assessed in that case. ${ }^{4}$

Conclusion: Levetiracetam is felt to be a well-tolerated medication. This case report of the youngest overdose of levetiracetam showed no acute or toxicity or delayed effects during a 1-year follow-up.

\section{A Case of Carbon Monoxide Neurotoxicity due to Smoking Hookah}

Angela Regina, Michael Nickas, Jakub Bartnik St Barnabas Hospital, Bronx, NY, USA

Introduction: Carbon monoxide (CO) poisoning is a common issue during the winter, due to the use of heating devices. ${ }^{1-3}$ A less common etiology that should be considered year round is from smoking Hookah. There is a growing popularity for this social past time especially in the younger population. $^{4-7}$

We report a case of $\mathrm{CO}$ neurotoxicity, which required hyperbaric oxygen therapy (HBO) after smoking Hookah.

Case: A 34-year-old Hispanic woman with past medical history of type I diabetes, latent tuberculosis, and hyperlipidemia was brought into an urban emergency department (ED) after a syncopal episode. The patient drank one beer and was smoking Hookah shortly before the event occurred. Her boyfriend reports that there was some shaking actively shortly after she passed out and afterward she was confused. At triage, the patient was afebrile, normotensive, breathing at 20 breaths per minute, slightly tachycardic at 102 beats per minute, and a point of care blood glucose was $373 \mathrm{mg} / \mathrm{dl}$. She was awake, alert, and oriented but complained of headache and chest pain. She had a grossly normal physical exam including no focal deficits, intact finger to nose, and a steady gait.

An EKG was interpreted as sinus rhythm at 95 beats per minute without signs of ischemia. A chest X-ray was obtained and was unremarkable. Troponin was $<0.01 \mathrm{ng} / \mathrm{ml}$. Lactic acid was $3.2 \mathrm{mmol} / \mathrm{L}$. Carboxyhemoglobin was $20.2 \%$.

The patient was already on $100 \%$ oxygen via nonrebreather mask and once the $\mathrm{CO}$ level was obtained, the local hyperbaric center was contacted and patient was transferred.

Discussion: CO poisoning accounts for over 20,000 ED visits per year and more than 4000 hospitalizations, with mortality of unintentional poisoning approximately 400 per year. $^{3}$

There have been cases reported internationally of similar cases to the above but not as many in the United States. A literature review found only one reported case in the US..$^{8-15}$

Conclusion: $\mathrm{CO}$ poisoning should not be forgotten about in syncope or seizure in the setting of possible inhalation exposure. Hookah inhalation 
may not be the first thing to come to mind, but it should be on one's differential as a reason for $\mathrm{CO}$ toxicity.

\section{Utilization of Informatics Tools to Mechanistically Analyze Drugs Associated with Serotonin Syndrome}

Rebecca Racz, Keith Burkhart

Food and Drug Administration, Silver Spring, MD, USA

Background: Serotonin syndrome (SS) is often a drug-drug interaction that results in enhanced serotonin neurotransmission by acting through different drug protein targets/pathways.

Research Question: Second-generation antipsychotics (SGAs) and concomitant drugs highly associated with SS and their molecular protein targets were evaluated to explore molecular protein targets and mechanisms for developing SS.

Methods: SGAs were data mined in FAERS to identify the concomitant drugs taken with SGAs and disproportionally associated with SS. Bioinformatics and cheminformatics tools were used to further mechanistically evaluate disproportionality of four classes of drugs. A bioinformatics tool data mines public FAERS for individual drugs, drug combinations, and drug targets for disproportionality using Proportional Reporting Ratio (PRR) scores. A cheminformatics tool evaluates potential unknown off-target binding. Literature searches and case analyses followed to further analyze signals, associations, and mechanisms.

Results: SGAs are disproportionally associated with SS (N: 1075, PRR: 4.59). Many serotonergic receptors were associated with $\mathrm{SS} ; 5-\mathrm{HT}_{2 \mathrm{~A}}(\mathrm{~N}$ : 916, PRR: 7.90), 5- $\mathrm{HT}_{1 \mathrm{~A}}$ agonism (N: 600, PRR: 8.73), and 5- $\mathrm{HT}_{2 \mathrm{C}}$ antagonism (N: 186, PRR: 15.07). Benzodiazepines were highly associated with SS (N: 1188, PRR: 4.83). Alprazolam and clonazepam were 2 of the top 20 concomitant medications with SGAs in 1075 SS cases (alprazolam concomitant N: 66, PRR: 4.28; clonazepam concomitant $\mathrm{N}$ : 133, PRR: 10.75). Cholinesterase inhibition (N: 120, PRR: 3.56) was found to be significantly associated with SS. N-methyl-D-aspartate (NMDA) antagonism was associated with SS (N: 219, PRR: 5.07). Pregabalin was predicted to bind to the NMDA receptor.

Discussion: Strong evidence exists for an association of SGAs and SS likely via two potential mechanisms of action found in literature: $5-\mathrm{HT}_{1 \mathrm{~A}}$ upregulation via $5-\mathrm{HT}_{2 \mathrm{~A}}$ antagonism and partial agonism at $5-\mathrm{HT}_{1 \mathrm{~A}}$. Some benzodiazepines may enhance serotonin activity via several mechanisms, including decreasing serotonin metabolism and increasing 5HT receptor reactivity, causing concern over their use for SS treatment. By increasing acetylcholine, cholinesterase inhibitors may have opposing serotonergic action through the muscarinic (decrease serotonin) and nicotinic (increase serotonin) receptors, while predicted pregabalin NMDA binding may increase serotonin neurotransmission.

Conclusions: SGAs, benzodiazepines (alprazolam and clonazepam), cholinesterase inhibitors, and pregabalin were found to be disproportionately associated with SS via various mechanistic pathways/targets.

\section{Retrospective Case Series of Buprenorphine Toxicity in the Pediatric Patient}

Todd Phillips ${ }^{1,2}$, Joshua Shulman ${ }^{2}$, Michael Lynch ${ }^{2}$

${ }^{1}$ John Peter Smith Hospital, Fort Worth, TX, USA

${ }^{2}$ University of Pittsburgh, Pittsburgh, PA, USA

Background: Buprenorphine is a partial mu-opioid agonist that is commonly prescribed for opioid addiction maintenance. Increasing utilization has been associated with more accidental pediatric ingestions. In adults, a ceiling effect on respiratory depression has been observed, but has not been fully evaluated in children.

Hypothesis: Buprenorphine causes significant toxicity in accidental pediatric ingestions which may be reversed by naloxone.

Methods: Pediatric patients less than 6 years old who presented to a tertiary care pediatric hospital and were evaluated by a medical toxicologist over a 5-year period were reviewed. Demographic information, reported dose, time to symptom onset, need for naloxone, and clinical respiratory information were obtained from the medical record.

Results: Thirty-six patients were enrolled. Mean age was 2.0 years $(0.6-$ 4.6). Evidence of toxicity was observed in 33 patients. Symptomatic patients demonstrated sedation $(100 \%)$, miosis $(76 \%)$, and dystaxia (39\%). Fourteen (42\%) patients had respiratory compromise including hypoventilation (57\%), need for oxygen supplementation (64\%), and/or need for intubation (14\%). Twenty-three patients had a well-defined time of ingestion. In these individuals, mean time to onset of symptoms was $1.3 \mathrm{~h}(0.3-4.0)$. Five patients did not have clear time of ingestion. Five others were known to be less than $4 \mathrm{~h}$, but a specific time of ingestion could not be established. Intravenous naloxone was utilized in $17(52 \%)$ patients. Forty-seven percent of these patients had complete resolution of their symptoms after only one dose. Five (29\%) patients required multiple doses of naloxone to return to baseline. Four (24\%) patients did not have any effect with the antidotal therapy.

Discussion: Due to the high incidence of respiratory depression, the authors recommend all children under 6 years of age with possible buprenorphine ingestion to receive an immediate medical evaluation. An observation period of $6 \mathrm{~h}$, as previously recommended, remains an adequate observation period if no symptoms develop prior to discharge. Should symptoms develop, the patient should be observed until these resolve. Naloxone administration has some antidotal activity; however, resistance to naloxone is observed.

Conclusion: Buprenorphine toxicity in the pediatric population has a high rate of respiratory depression and naloxone rescue therapy has varying effects.

\section{Pancreatic Pseudocyst Due to Acute Valproic Acid Overdose}

Kaitlin Patrick, Jenna Jarriel, Michelle Hieger Wellspan Health, York Hospital, York, PA, USA

Background: Valproic acid is a frequently used anticonvulsant and mood stabilizer. Acute overdose of the drug is common, with 2998 single exposures documented in the United States in 2014. A black box label has been placed on valproic acid warning of pancreatitis. Pancreatitis after acute overdose is documented, but pancreatic pseudocysts are rarely reported.

Research Question: Can pancreatic pseudocysts occur with acute valproic acid overdose?

Methods: This is a single case report. A 37-year-old male with history of seizures and bipolar disorder presented with altered mental status, abdominal pain, and vomiting. The patient took 50 tabs of valproic acid $500 \mathrm{mg}$. Initial vital signs were blood pressure $121 / 85 \mathrm{mmHg}$, heart rate 104 beats per minute, temperature $36.8^{\circ} \mathrm{C}$, respiratory rate 18 breaths per minute, and $97 \%$ oxygen saturation on room air. The patient was somnolent, followed simple commands, had epigastric abdominal tenderness, and otherwise an unremarkable exam.

Results: The initial valproic acid concentration was $>450$ (50-100 mcg/ $\mathrm{mL})$ and ammonia was $137(17-60 \mathrm{mcmol} / \mathrm{L})$. Lipase and amylase levels were 2134 (11-82 U/L) and 582 (29-103 U/L), respectively. Transaminases and bilirubin were normal. Levocarnitine $6 \mathrm{~g}$ every $8 \mathrm{~h}$ was initiated. Within $24 \mathrm{~h}$, he became more alert and complained of abdominal pain. A computed tomography (CT) scan of the abdomen and pelvis showed pancreatic edema with prominent diffuse peripancreatic inflammatory stranding. After 3 days, his valproic acid, ammonia, and lipase levels normalized. One week later (still hospitalized), the patient developed a temperature of $38.6^{\circ} \mathrm{C}$. A repeat $\mathrm{CT}$ of the abdomen and pelvis showed a pancreatic pseudocyst. The aspirated fluid collection did not show an infectious etiology. Since his hospitalization, the patient has been evaluated 4 times in 6 months for recurrent pancreatitis with pseudocysts.

Discussion: Patients can develop the rare complication of pancreatic pseudocysts from acute valproic acid toxicity. This patient developed pancreatitis with pseudocyst within 1 week of an acute ingestion of valproic acid. 
Conclusion: Lipase levels with mild abdominal pain after resolution of initial pancreatitis may prove beneficial for early treatment of recurrent pancreatitis, with consideration of avoidance of valproic acid in the future if a patient develops recurrent pancreatitis.

\section{Comparing Online Poison Triage System to Poison Control Center}

Yakisha Partee $^{1}$, David Liss ${ }^{1}$, Michael Mullins ${ }^{1}$, Evan Schwarz ${ }^{1}$, Julie Weber $^{2}$, Rebecca Tominack ${ }^{2}$

${ }^{1}$ Division of Emergency Medicine, Washington University, St. Louis, MO, USA

${ }^{2}$ Missouri Poison Center at SSM Cardinal Glennon Children's Medical Center, St. Louis, MO, USA

Background: A new web-based poison triage system (webPOISONCONTROL®) allows users to access poison control information and guidance through the internet rather than calling poison control centers (PCC). We seek to compare dispositions through this webbased triage system (WBTS) to PCC.

Research Question: Are the dispositions provided through WBTS concordant with dispositions given through PCC?

Methods: This is a consecutive sample study. We reviewed calls to a PCC for oral ingestions during 2015 with disposition to remain at home. We then simulated each of these cases through the WBTS to compare disposition to the actual disposition resulting from live contact through a PCC. We classified the WBTS outcomes as referral to ED, referral to PCC, or remain at home. We classified WBTS outcomes leading to direct referral to ED as discrepant with PCC outcomes. We classified WBTS outcomes leading to referral to PCC or remain at home as concordant with PCC outcomes.

Results: We reviewed 206 total calls of which 103 were defined as moderate outcome and 103 were defined as minor outcome. Of 206 total dispositions through the WBTS, $26(13 \%)$ were to "remain at home," $180(87 \%)$ were "referral to PCC," and none were "referral to ED."

Discussion: The minority of WBTS outcomes of "remain at home" were concordant with outcomes of live calls to the PCC. A majority of WBTS outcomes were "referral to PCC" for multiple reasons and were, by definition, concordant. No WBTS encounter in this set led to a discordant outcome of "direct referral to the ED."

Conclusion: As nearly $90 \%$ of callers were referred to the PCC, the WBTS could not replace the PCC and it is questionable how much it improves our current system.

\section{You Must-ard Be Kidding Me: A Case of Sulfur Mustard Exposure}

Jenna Otter, Alveena Dawood, Joseph D’Orazio Temple University Hospital, Philadelphia, PA, USA

Background: Sulfur mustard is a vesicant chemical warfare agent used in World War I. Today, exposure to sulfur mustard is a rare event. Exposures sporadically occur from abandoned artillery shells in coastal waters. We present a case of sulfur mustard exposure in a fisherman off the coast of New Jersey.

Methods: A 40-year-old male fisherman was dredging for clams on a commercial boat when he pulled up an old artillery shell. With his right arm, he threw the shell into the water without incident. Shortly after, he developed pain and blisters along his right hand and forearm. Over the next few hours, the pain worsened until he presented to an emergency department and subsequently transferred for burn care. The patient denied shortness of breath, cough, and eye and throat irritation. Vital signs and physical exam were normal except for a partial thickness circumferential second-degree burn from the hand to the elbow with ruptured blisters diffusely. The patient was treated with silver sulfadiazine and foam dressing, opioid pain control, and occupational therapy. The patient required a prolonged hospital stay with split thickness graft of the entire right forearm 20 days after admission. Upon follow-up, the patient was improving without complications.

Discussion: Sulfur mustard (dichlorodiethyl sulfide) is a viscous liquid used as a blistering agent in chemical warfare. Until the 1970s, the dumping of unwanted munitions, including sulfur mustard artillery shells, into coastal waters was a standard practice. While sulfur mustard production in the US ceased in 1968, there are sporadic reports of sulfur mustard exposure to military personnel and civilians from discarded artillery shells. Cutaneous exposure to the yellowbrown liquid commonly leads to a significant cutaneous blistering injury that may take several months to heal and may result in considerable permanent cosmetic and functional impairment. The longterm effects of sulfur mustard exposure include an increased risk for various cancers, chronic respiratory problems, and post-traumatic stress disorder.

Conclusion: We present a rare case of sulfur mustard exposure from a discarded artillery shell off the coast of New Jersey causing to significant blistering that required skin grafting.

77. Malignant Catatonia Mimicking Serotonin Syndrome: Two Cases Treated Successfully with Electroconvulsive Therapy

Laura Ortiz, Louise Kao, Laura Tormoehlen, Dan Rusyniak Indiana University School of Medicine, Indianapolis, IN, USA

Background: Serotonin syndrome (SS) and malignant catatonia (MC) have overlapping symptoms and are challenging to differentiate.

Hypothesis: Considering the diagnosis of MC in patients failing typical therapy for SS can lead to different treatment strategies.

Methods: A two patient chart review.

Results: Case 1: A 46-year-old male presented to the emergency department (ED) from a psychiatric facility. He was confused, tremulous, with clonus and rigidity in all extremities. His medications included lithium, escitalopram, trazodone, and paliperidone without recent changes. Initial vitals were unremarkable except temperature $99.5^{\circ} \mathrm{F}$. He was treated for suspected SS with lorazepam and cyproheptadine $8 \mathrm{mg}$ q6h. On hospital day (HD) \#3, he developed fever (Tmax 101.8), tachycardia, and delirium, and required intubation. Fever, rigidity, and tachycardia persisted. On HD \#10, the diagnosis of MC was entertained. Electroconvulsive therapy (ECT) started on HD \#10 and his mental status and rigidity improved. He received 10 ECT treatments and scheduled lorazepam and was discharged on HD \#30 at his baseline neurological state.

Case 2: A 24-year-old male presented to the ED from jail with headache and fevers. His home medications included quetiapine, citalopram, and clonazepam, without recent changes. His initial vital signs were temperature $100.5^{\circ} \mathrm{F}$, heart rate 118 , blood pressure $160 / 90$, and respiratory rate 24. On exam, he had rigidity and clonus in all extremities. Over $12 \mathrm{~h}$, he became increasingly febrile (Tmax $\left.107^{\circ} \mathrm{F}\right)$, tachycardic, and delirious requiring intubation. He was treated for SS with lorazepam and cyproheptadine $12 \mathrm{mg}$ then $2 \mathrm{mg} \mathrm{q} 2 \mathrm{~h}$. He remained febrile and rigid despite escalating sedation with lorazepam, propofol, and pentobarbital. On HD \#14, the diagnosis of MC was entertained and ECT was started with clinical improvement. He received 5 ECT treatments and was extubated on HD \#20. He was discharged on HD \#38 at his baseline neurological state.

Discussion: MC shares significant symptom overlap with SS including altered mental status, rigidity, and autonomic dysfunction. However, treatment strategies differ between these entities.

Conclusion: We present two cases of SS mimicking MC, which were successfully treated with ECT. MC should be considered in the differential diagnosis with SS, as appropriate treatment can lead to successful recovery. 
78. Hydroxocobalamin Extravastation, Progression, and Resolution in a House Fire Victim

\author{
Supa Niruntarai $^{1}$, Ross Sullivan ${ }^{1}$, William Eggleston ${ }^{1,2}$ \\ ${ }^{T}$ SUNY Upstate Medical University, Department of Emergency Medicine, \\ Syracuse, NY, USA \\ ${ }^{2}$ Upstate New York Poison Center, Syracuse, NY, USA
}

Background: Hydroxocobalamin is an antidote for cyanide toxicity administered intravenously. Extravasation of hydroxocobalamin is not reported.

Hypothesis: Hydroxocobalamin is a benign medication that does not produce significant tissue injury after extravasation.

Methods: This is a single patient chart review. An 89-year-old female presented to an emergency department unresponsive after being exposed to smoke in her apartment secondary to a fire in the adjacent apartment. The patient was bradycardic and hypotensive. Smoke inhalation injury and cyanide toxicity were suspected. She was intubated and intravenous hydroxocobalamin was administered by EMS. On arrival, significant physical exam findings included dark purple discoloration and swelling at the infusion site over the left forearm extending to the dorsum of the left hand without impaired tissue perfusion. No skin breakdown or necrosis was noted. No other medications were administered in the catheter at the extravasation site.

Results: The patient's left arm was elevated and warm compresses were applied to the site to manage the extravasation. She had orange discoloration of the urine that persisted for 6 days after hydroxocobalamin administration. On hospital day 7, the patient's skin discoloration and swelling had significantly improved and cleared. Her left forearm was well perfused. She denied pain, tingling, and weakness of the left arm and hand.

Discussion: Extravasation of intravenous medications can result in tissue injuries varying from local swelling and redness to necrosis and compartment syndrome. Tissue injuries following extravasation depend on a number of drug-specific factors, including the active ingredients, diluents, concentration, and volume extravasated. Treatment of extravasation injuries varies from conservative care to surgical intervention. Hydroxocobalamin has a favorable safety profile and causes only minor adverse effects including red discoloration and colorimetric laboratory assay interferences. However, the appropriate management of hydroxocobalamin extravasation is not known. Our case demonstrated mild local swelling and tissue discoloration following hydroxocobalamin extravasation. Both resolved by hospital day 7 without any significant complications.

Conclusion: Extravasation of hydroxocobalamin is unlikely to cause serious local tissue injuries or long-term sequelae. Elevation and warm compresses are likely sufficient for managing the extravasation.

\section{Naloxone Access Among an Urban Population of Opioid Abusers}

Jenna Nikolaides ${ }^{1}$, Lum Rizvanolli ${ }^{2}$, Michael Rozum ${ }^{2,3}$, Steven Aks ${ }^{1,2}$

${ }^{I}$ Toxikon Consortium, Chicago, IL, USA

${ }^{2}$ Cook County Health and Hospital System, Chicago, IL, USA

${ }^{3}$ Rush Medical College, Chicago, IL, USA

Background: Take-home naloxone is one method of curbing the mortality associated with the opioid epidemic. It is unclear if some opioid users have more access to naloxone than others.

Research Question: What is the access to naloxone in an urban emergency department (ED) and does naloxone awareness and access differ between people who abuse different types of opioids?

Methods: A convenience sample of 101 patients was surveyed in an urban academic ED, over a 6-month period (April to October, 2016). A 14-question survey was drafted, validated by a panel of medical toxicologists, piloted, and then edited for clarity. The survey was then administered to ED patients by standardized trained research assistants. Inclusion criteria were opioid abuse within the previous 3 months and age greater than 18 years. Exclusion criteria were acute intoxication and inability to consent, incarceration, involuntary psychiatric admissions, and those taking opioids as prescribed were excluded. Data was analyzed using descriptive statistics. This study received IRB exempt status.

Results: The average age of survey responders was 47.3 years old; $72 \%$ were male; $57 \%$ black, $31 \%$ white; $15 \%$ Hispanic. Current method of opioid abuse: $38 \%$ inject heroin, $72 \%$ snort heroin, $8 \%$ abuse prescription pills. When asked about naloxone, $55 \%$ had heard of it and $22 \%$ had access to it at some point.

Of the 54/101 responders with a history of injecting heroin, $76 \%(41 / 54)$ had heard of naloxone, and 39\% (21/54) had access to it at some point. Of the $47 / 101$ who had never injected heroin, only $32 \%(15 / 47)$ had heard of naloxone, and $2 \%(1 / 47)$ had access to it.

All 22 responders with access to naloxone had gotten it for free or from someone they knew; 7 reported use on themselves, and 10 reported use on someone else. In total, 43 naloxone reversals were reported.

Discussion: Snorting heroin was the most common method of opioid abuse. However, heroin injectors had better naloxone awareness and access. Those that had naloxone reported a high rate of use. Limitations include the survey format and its singlecenter urban setting.

Conclusion: Naloxone distribution appears to be missing certain populations of opioid abusers.

\section{Fatal Intoxication of Twin Girls After Ingestion of Topical $2 \%$} Viscous Lidocaine

Aimee Nefcy, Eric Malone, Andrew King, Cynthia Aaron

Wayne State University / Children's Hospital of Michigan Regional Poison Control Center, Detroit, MI, USA

Background: Lidocaine is a well-known neurocardiotoxin. Significant toxicity from oral ingestion is uncommon in the pediatric age group.

Hypothesis: Oral ingestion of viscous lidocaine can result in significant toxicity and death in pediatric patients.

Case Report: Twin 13-month-old girls who were both being treated for teething pain by their pediatrician with topical lidocaine presented separately but within $48 \mathrm{~h}$ of each other with generalized tonic-clonic seizures followed by cardiac arrest. After prolonged resuscitation and return of spontaneous circulation, twin A was transferred to the pediatric ICU but without cortical neurologic activity. Her parents were visiting her when twin $B$ arrived in the ER with a near-identical presentation. However, twin B was unable to be resuscitated and expired. The family were recent immigrants to the US. Extensive home investigation revealed the empty bottle of $2 \%$ viscous lidocaine. Serum and urine testing from both children later revealed toxic concentrations of lidocaine and monoethylglycinexylidide. Further questioning of the family eventually suggested that their teenaged brother may have confused the lidocaine with acetaminophen and had been administering the medication in their milk bottles because of incessant crying.

Discussion: Viscous lidocaine remains a commonly prescribed medication for symptomatic relief of multiple ailments. Potential toxicity of ingested viscous lidocaine is previously poorly described. This tragic case demonstrates the potential for severe toxicity of this commonly prescribed medication.

Conclusion: While uncommon, systemic toxicity from orally ingested viscous lidocaine is a potentially devastating complication of overdose, especially in cases of poor caretaker health literacy. Clinicians should be aware of the potential for intoxication and reserve topical anesthetics for situations where they are absolutely necessary. 


\section{Artificially Elevated Serum Creatinine after Nitromethane} Ingestion

Anita Mudan ${ }^{1}$, Jeanmarie Perrone ${ }^{1,2}$, Holly Thompson ${ }^{2}$, Jeanette Trella ${ }^{2}$, Diane Calello ${ }^{3}$

${ }^{T}$ Hospital of the University of Pennsylvania, Philadelphia, PA, USA

${ }^{2}$ The Poison Control Center at The Children's Hospital of Philadelphia, Philadelphia, PA, USA

${ }^{3}$ New Jersey Poison Information and Education System, NJ, USA

Background: Nitromethane is a colorless solvent, used as a racecar fuel, and is often found in combination with methanol and a lubricant. The oxygen content in nitromethane promotes efficient combustion and increases horsepower. After pediatric ingestion of nitromethane-containing racecar fuel, methanol poisoning is typically the greatest concern; however, laboratory interference from nitromethane may confuse care.

Case Report: A 20-month-old boy was found drinking fuel, believed to be "methanol," intended for radio-controlled cars. Initial symptoms included drooling and emesis. Vital signs were HR 97/min, BP 84/ $54 \mathrm{mmHg}$, RR 24/min, and $\mathrm{SpO}_{2} 99 \%$. His exam was only notable for oropharyngeal erythema. Initial serum/blood laboratory studies included bicarbonate $19 \mathrm{mEq} / \mathrm{L}$ with anion gap of $18 \mathrm{mEq} / \mathrm{L}$, osmolal gap of $3.5 \mathrm{mmol} / \mathrm{kg}$, and creatinine of $2.8 \mathrm{mg} / \mathrm{dL}$ (repeated $-2.4 \mathrm{mg} / \mathrm{dL}$ ). A renal ultrasound was normal. Given the elevated anion gap and concern for renal failure, fomepizole was adminstered and he was transferred to a tertiary care hospital. The fuel was identified as $S \& W$ Standard $R C C a r$ Fuel containing nitromethane, methanol, and castor oil. He was monitored overnight, serum methanol and ethylene glycol concentrations were undetectable, and he was discharged home.

Discussion: We present a case of a falsely elevated serum creatinine after nitromethane ingestion. Serum creatinine is commonly measured by one of two methods. In the Jaffe Reaction, creatinine reacts with alkaline sodium picrate to form a red-orange chromophore which absorbs light in the range of 470-550 nm on spectroscopy. The active methylene group on nitromethane also reacts with alkaline sodium picrate to form a chromophore which absorbs light at the same wavelengths. Thus, serum creatinine measurements via the Jaffe Reaction are expected to be falsely elevated in the presence of nitromethane. This false reading can be avoided by utilizing the three-step enzymatic method that ultimately produces hydrogen peroxide, which is measured and accurately correlates with serum creatinine even in the presence of nitromethane. The two hospitals involved in this boy's care used different methods for creatinine measurement.

Conclusion: Health care providers are once again alerted that nitromethane is a chemical known to interfere with the Jaffe Reaction method of creatinine measurement. Recognition can lead to more appropriate delivery of medical care.

82. Muscimol and ibotenic acid containing mushrooms exposures: US National Poison Data System 2001-2011

Michael Moss ${ }^{1,2}$, Robert Hendrickson ${ }^{1,2}$, Benjamin Hatten ${ }^{3,4}$

${ }^{T}$ Oregon Health \& Science University, Portland, OR, USA

${ }^{2}$ Oregon Poison Center, Portland, OR, USA

${ }^{3}$ University of Colorado School of Medicine, Aurora, CO, USA

${ }^{4}$ Rocky Mountain Poison and Drug Center, Denver, CO, USA

Background: There are several species of muscimol/ibotenic acidcontaining mushrooms in the US including Amanita muscaria and Amanita pantherina. Data on exposure to these mushrooms is limited to case reports and small case series.

Research Question: What are the demographics, distribution, frequency, and outcomes of exposure to muscimol/ibotenic acid-containing mushrooms in the U.S.?

Methods: Retrospective review of NPDS muscimol-containing mushroom exposures from 2001 to 2011. Descriptive statistics were used to analyze the data.
Results: There were 66 pediatric exposures ( $<6$ years) and 414 cases $>6$ years, or of unknown age. Three deaths were reported and all were intentional adult exposures. Thirty-five cases resulted in a major effect with 26 of these cited as intentional ingestions.

Observed effects were none (14\%), minor (20\%), moderate (39\%), major (7\%), and unknown (20\%). Other outcomes were GI symptoms (29\%), seizures (4\%), and intubation (9\%). Dispositions included managed onsite $(18 \%)$, treated and released (39\%), admitted to the hospital $(32 \%)$, and remainder unknown.

Compared to A. pantherina, A. muscaria ingestions were less likely to require critical care (20 vs $28 \%$ ), less likely to have major or moderate effects ( 30 vs $15 \%$ ), and to be intentional (66 vs $44 \%$ ). Pediatric exposures accounted for $13 \%$ of cases overall, but with regional variability ( $>70 \%$ of Alaskan cases were $<6$ years old). Only 13 (20\%) pediatric exposures developed symptoms. Three regions of the country accounted for the majority of exposures: Pacific West (31\%), Northeast (20\%), and East North Central (17\%). Map of ingestions will be presented. There was marked seasonal variation with a predilection for fall (50\% AugOct. $81 \%$ July-Dec). Seventy-four percent of cases were from urban areas and $24 \%$ from rural areas.

Discussion: The majority of reported cases are intentional adult ingestions of A. muscaria or accidental adult ingestions of $A$. pantherina with $>20 \%$ receiving critical care. A. pantherina exposures are more often unintentional with more severe effects, perhaps related to unintentional foraging compared to the distinctive A. muscaria. GI symptoms are relatively common. Pediatric exposures are less frequent and less likely to develop severe symptoms.

Conclusion: Muscimol-containing mushroom exposures in children ( $<6$ years) are largely asymptomatic with rare severe symptoms and A. pantherina causing more severe toxicity than A. muscaria.

83. Toxicity of Ibotenic Acid and Muscimol Containing Mushrooms Reported to a Regional Poison Control Center From 2002 to 2016

Michael Moss ${ }^{1,2}$, Robert Hendrickson ${ }^{1,2}$

${ }^{T}$ Oregon Health and Science University, Portland, OR, USA, ${ }^{2}$ Oregon Poison Center, Portland, OR, USA.

Background: Amanita muscaria (AM) and Amanita pantherina (AP) contain ibotenic acid and muscimol and may cause both excitatory and sedating symptoms. A "typical" syndrome of accidental ingestion with CNS depression in adults and CNS excitation in children and a paucity of GI symptoms or respiratory depression are based on relatively few reported cases with these mushrooms in North America.

Research Question: What are the clinical effects of ibotenic acid/ muscimol containing mushroom toxicity?

Methods: Retrospective review of ingestions of ibotenic acid/muscimol containing mushrooms reported to a regional poison center from 2002 to 2016. Cases were included if identification was made by a mycologist or if AM was described as a red/orange mushroom with white spots.

Results: Thirty-five cases met inclusion criteria. There were 24 cases of $\mathrm{AM}, 10 \mathrm{AP}$, and $1 \mathrm{~A}$. aprica. Reason for ingestion included foraging (12), recreational (5), accidental (12), therapeutic (1), and self-harm (1). Of the accidental pediatric ingestions, $4(25 \%)$ were symptomatic. None of the children with a symptomatic ingestion of AM required admission. A 3year-old male who ingested AP developed vomiting, agitation, and lethargy. He was intubated and had a 3-day ICU stay. There were 25 symptomatic patients in total. All but one developed symptoms within $6 \mathrm{~h}$. Duration of symptoms was $<6 \mathrm{~h}(6,24 \%), 6-24 \mathrm{~h}(15,60 \%),>24 \mathrm{~h}(1$, $4 \%)$, and unreported $(3,12 \%)$. Ingestions of AP were more symptomatic than AM with regard to the presence of gastrointestinal symptoms ( $89 \mathrm{vs}$ $60 \%$ ), CNS depression (78 vs 60\%), and CNS excitation (89 vs $40 \%$ ), respectively. Seven patients were given benzodiazepines. Seventeen received activated charcoal. Five (20\% of symptomatic) were intubated. None experienced hypotension, seizures, acute kidney injury, or hepatotoxicity. No deaths were reported. 
Discussion: Ingestion of ibotenic acid/muscimol containing mushrooms often produces a syndrome with GI upset, CNS excitation, and CNS depression either alone or in combination. Ingestion of AP was associated with a higher rate of symptoms compared to AM.

Conclusion: In contrast to previous reports, ibotenic acid/muscimol mushroom poisoning was commonly reported with intentional ingestions and associated a high rate of GI symptoms, no seizures, and several intubations.

\section{Pharmacogenetic Determinants of Bone Toxicity Among Children Treated With Chemotherapy for Acute Leukemia}

Yaron Finkelstein $^{1}$, Traci M Blonquist ${ }^{2}$, Veena Vijayanathan ${ }^{3}$, Kristen E. Stevenson $^{2}$, Donna S. Stevenson ${ }^{2}$, Lewis B Silverman ${ }^{2,5}$, Lynda M. Vrooman $^{4,5}$, Stephen E. Sallan ${ }^{4,5}$, Peter D Cole ${ }^{3}$

${ }^{1}$ Hospital for Sick Children, University of Toronto, Toronto, ON, Canada

${ }^{2}$ Dana Farber Cancer Institute, Boston, MA, USA

${ }^{3}$ Albert Einstein College of Medicine, Bronx, NY, USA

${ }^{4}$ Boston Children's Hospital, Boston, MA, USA

${ }^{5}$ Harvard Medical School, Boston, MA, USA

Background: Bone fractures and osteonecrosis frequently complicate therapy for childhood acute lymphoblastic leukemia (ALL). Bone toxicity has been associated with exposure to corticosteroids and methotrexate and age greater than 10 years.

Study Question: Are common pharmacogenetic polymorphisms associated with bone toxicity in children during treatment for acute leukemia? Methods: Six hundred fifteen out of 794 children with acute leukemia enrolled on DFCI ALL Consortium protocol 05-001 (NCT00400946) met eligibility criteria for inclusion in this analysis. Nineteen candidate polymorphisms were selected a priori, targeting genes related to glucocorticoid metabolism, oxidative damage, and folate physiology. Polymorphisms were genotyped using either PCR-based allelic discrimination or PCR product length analysis.

Results: Twenty percent of subjects were homozygous for two 28 bp repeats $(2 \mathrm{R} / 2 \mathrm{R})$ within the $5^{\prime}$ untranslated region of the gene for thymidylate synthase (TS). This $2 \mathrm{R} / 2 \mathrm{R}$ genotype was associated with increased risk of osteonecrosis among children younger than 10 years at diagnosis (multivariable hazard ratio 2.71 ; 95\% CI $1.23-$ $5.95 ; p=0.013$ ) and with bone fracture among children $\geq 10$ years (multivariable HR 2.10; 95\% CI 1.11-3.96; $p=0.022$ ). No significant association was observed between TS genotype and RBC folate, RBC methotrexate, or relapse risk.

Conclusions: A common genetic variant is associated with increased risk of osteonecrosis among children younger than 10 years treated for acute leukemia and with bone fractures among older children. These findings suggest that children and adolescents with the 2R/2R TS genotype should be closely monitored for the development of bone toxicity during therapy for ALL and support a clinical trial testing the efficacy of protective interventions specifically in this vulnerable population.

\section{Buprenorphine Treatment of Opioid Dependence for Patients Hospitalized With Infective Endocarditis}

Aaron Fields, Timothy Wiegand

University of Rochester Medical Center, Rochester, NY, USA

Introduction: Infective endocarditis (IE) is a life-threatening infection involving the valves of the heart, with in-hospital mortality between 15 and $30 \%[1,2]$. Treatment typically includes 4-6 weeks of antibiotics, often requiring surgical repair or replacement of damaged valves [3-5]. Intravenous drug use (IVDU) is a major risk factor for IE, leading to a 10fold higher rate of death or re-operation between 3 and 6 months following an index operation for endocarditis [5]. In opioid-dependent individuals, treatment with buprenorphine has been shown to reduce IVDU and improve engagement in treatment.
Research Question: Early initiation of buprenorphine during hospitalization for IE improves patient satisfaction and adherence to treatment.

Methods: Retrospective chart review of three opioid-dependent patients during 2015-16 who were started on sublingual buprenorphine during hospitalization for IE secondary to IVDU.

Case Discussion: Case 1-A 33-year-old opioid-dependent female with intravenous heroin and cocaine abuse, 2 previous episodes of IE, and valve replacement/repair is hospitalized for recurrent IE. SL buprenorphine was started during her hospitalization.

Case 2-A 33-year-old female with intravenous heroin and cocaine abuse is hospitalized for IE and started on SL buprenorphine while receiving antibiotics.

Case 3-A 30-year-old opioid-dependent male with prior IE from intravenous heroin use is re-hospitalized for IE and undergoes valve replacement. SL buprenorphine was started during hospitalization.

Duration of hospitalization ranged from 45 to 57 days. All patients completed 6-week antibiotic courses and were successfully linked to ongoing addiction treatment. The buprenorphine and addiction support were managed by the toxicology consultation service during the hospitalization.

Discussion: Previous studies of IVDU-associated endocarditis have shown poor outcomes. In our experience, rapid buprenorphine initiation during hospitalization has enabled patients to tolerate prolonged hospitalization without leaving AMA. Such prolonged hospitalization also represents an opportunity to engage the patient in other meaningful aspects of recovery such as peer counseling and 12-step programs. Although further research is needed, these findings are encouraging. This represents an opportunity for medical toxicology consult services to expand their role in the management of acute withdrawal and complications of addiction in hospitalized patients.

\section{Intentional Abuse of Loperamide: Exposure Trends Reported to US Poison Control Centers, 2011-2016}

Matthew Fernandez, Eric Nguyen, Jane Miloradovich, Jeanette Trella, Kevin Osterhoudt

The Poison Control Center at The Children's Hospital of Philadelphia, Philadelphia, PA, USA

Background: Loperamide is a peripherally acting mu-opioid agonist and is an over-the-counter anti-diarrheal in the United States. In high doses, loperamide can cross the blood-brain barrier to produce euphoria and is sometimes abused or used to curb withdrawal symptoms. In light of the ongoing opioid epidemic, we aimed to analyze the epidemiology of loperamide abuse as reported to the American Association of Poison Control Centers (AAPCC).

Research Question: Has the intentional abuse pattern of loperamide changed over the past half decade and is there evidence of harm?

Methods: A retrospective review of deidentified (IRB exempt) AAPCC data from January, 2011, to September, 2016, was performed. Singlesubstance human exposure cases involving loperamide, coded as intentional abuse, were extracted from the AAPCC National Poison Data System. Subjects were excluded if they had no age or gender data, or if they were miscoded.

Results: Two hundred forty-six cases were extracted and $17(7 \%)$ were excluded for insufficient data. Of the 229 cases coded as intentional loperamide abuse, $76 \%$ were male. The ages ranged from 13 to 87 years (mean 29 years, mode 19 years). From 2011 through 2013, the number of reports remained consistent with an average of 15/year (range 13-18); then a sharp increase was noted with 40 in 2014, 46 in 2015, and 97 in the first 9 months of 2016. Seven (3\%) deaths were directly or indirectly attributed to loperamide abuse. Major effects were coded in 40 patients $(17 \%)$, moderate effects in $71(31 \%)$, minor in $40(17 \%)$, and minimal or no effects in 36 (16\%); the outcomes were not coded for 34 patients $(15 \%)$. The majority of cases, 214 , were either enroute or referred to a health care facility. Known biases exist in the AAPCC database. 
Discussion: The data shows a marked increase in poison center calls related to intentional abuse of loperamide over time, almost sevenfold from 2011 to 2016, with a majority of cases being managed in a health care facility. This AAPCC data suggests that loperamide abuse is on the rise and is potentially dangerous. Pharmacists and other health care professionals should be alerted to this social phenomenon. Abuse deterrent strategies warrant careful consideration.

\section{Lady Stone Heart?}

Natalija Farrell ${ }^{1}$, Jason Hack ${ }^{2}$

${ }^{T}$ Rhode Island Hospital, Providence, RI, USA

${ }^{2}$ Alpert Medical School at Brown University, Providence, RI, USA

Background: Digoxin's inhibition of the sodium/potassium ATPase pump contributes to its therapeutic and toxic effects. The presence of hyperkalemia is predictive of mortality in acute digoxin toxicity cases, and the treatment of hyperkalemia with intravenous (IV) calcium in this setting is controversial.

Case Report: A 71-year-old female with a history of atrial fibrillation, pulmonary hypertension, and coronary artery disease presented to the ED after syncope. Her vital signs were normal and examination demonstrated generalized weakness, altered mental status, tremors, and abdominal pain. She was found to have an acute kidney injury [BUN $64 \mathrm{mg} / \mathrm{dL}, \mathrm{SCr}$ $1.43 \mathrm{mg} / \mathrm{dL}]$ and hyperkalemia [6.1 mEq/L]. ECG demonstrated T wave inversions and ST depressions, and an elevated troponin $[0.105 \mathrm{ng} / \mathrm{mL}]$. Head CT was unremarkable. She received insulin IV, kayexelate PO, and calcium gluconate IV for the hyperkalemia. Within minutes of receiving calcium, the patient had a transient episode of cardiac arrest that resolved without intervention and was subsequently found to have an elevated digoxin level [4.3 ng/mL]. Repeat ECG showed worsening ST depressions but down trending troponin $[0.086 \mathrm{ng} / \mathrm{mL}]$ and no chest pain. She received digoxin immune fab therapy and symptoms of digoxin toxicity resolved. There were no other complications during her hospitalization and she was discharged home when her renal function improved.

Discussion: The treatment of hyperkalemia with calcium IV in digoxin toxicity may result in a state of myocardial tetany or "stone heart" due to elevated intracellular calcium levels in the cardiac myocytes. Animal studies appear to disprove the "stone heart" theory. Historically, the few human case reports of "stone heart" were incomplete and the largest retrospective study to date did not find an increase in dysrhythmias or mortality when calcium was given for hyperkalemia in chronic digoxin toxicity cases. Our case suggests that calcium induced cardiac arrest in the setting of digoxin toxicity. The exogenous calcium administration likely worsened the digoxin toxicity's cytoplasmic hypercalcemia, which is associated with dysrhythmias.

Conclusion: This case highlights the need for caution when giving calcium IV in patients with digoxin toxicity who do not have ECG findings suggestive of hyperkalemia.

\section{Mortality Associated with Abuse of Novel Opioid, U47700}

Andrew Farkas, Joshua Shulman, Michael Lynch

University of Pittsburgh Medical Center, Pittsburgh, PA, USA

Background: U47700 is a synthetic opioid with approximately 7.5 times the potency of morphine in animal models. It is also an emerging drug of abuse which, as of November 2016, has been placed into Schedule 1 of the Controlled Substances Act by the DEA.

Hypothesis: U47700 is an emerging drug of abuse which produces an opioid toxidrome. Like other opioids, its use can cause fatal respiratory depression

Methods: This is a retrospective review of a case in which a patient presented with multiple separate instances of sedative-hypnotic toxicity. Hospital records were reviewed.

Case Report: A 30-year-old male with a history of substance use disorder, depression, and prior suicide attempts presented with somnolence, hypoxemia, and findings consistent with aspiration after a suspected polysubstance overdose. He was taken to a community hospital and required intubation for airway protection as well as norepinephrine for hypotension. He was transferred to a tertiary care center. Urine gas chromatography/mass spectrometry (GC/MS) demonstrated U47700, dextromethorphan, hydroxyzine, quetiapine, diphenhydramine, and paroxetine. The patient recovered and was extubated. He admitted to buying U47700 over the internet and ingesting it prior to hospitalization. His family member produced the substance, which consisted of dropper bottles marked "U47700 for research purposes only $20 \mathrm{mg}$ per $\mathrm{mL}$ ". They contained $5 \mathrm{~mL}$ of a clear, sticky fluid. The patient was transferred to inpatient psychiatry after a 5-day hospitalization and was discharged home 8 days after that. Two weeks later, the patient was found dead of a suspected U47700 overdose according to a DEA report, though final medical examiner determination is pending.

Discussion: We present a case of a patient who is suspected to have died as a result of U47700 overdose. The substance was detectable on urine GC/MS. The patient reportedly obtained the substance via an internet website.

Conclusion: U47700 is a novel opioid with greater potency than morphine and is available for home delivery from internet sources. It can be identified in biological specimens via urine GC/MS. Deaths from U47700 toxicity have been reported.

\section{Clinical Manifestations of 3-Methoxy-Phencyclidine Intoxication}

Andrew Farkas, Joshua Shulman, Joseph Yanta, Anthony Pizon, Michael Lynch University of Pittsburgh Medical Center, Pittsburgh, PA, USA

Background: 3-Methoxy phencyclidine (3-MEO-PCP), a designer drug sold online as a "research chemical," is a high-affinity NMDA antagonist that is emerging as a novel drug of abuse.

Hypothesis: 3-MEO-PCP toxicity is consistent with other dissociative agents, but may be more sedating.

Methods: This is a retrospective review of a consecutive series of three patients with suspected 3-MEO PCP intoxication. Hospital records, including comprehensive urine drug screen (CUDS), and other information in the patient record were examined.

Case Reports: I: A 25-year-old male with a history of substance use disorder was brought to the emergency department with altered mental status and suspected intoxication. On arrival, he was noted to be somnolent with nystagmus, borderline tachycardia, and otherwise had unremarkable vital signs. His mental status resolved over the course of a 48 -h hospital stay, and he was discharged home. Urine gas chromatography/ mass spectrometry analysis (GC/MS) showed 3-MEO-PCP and flubromazepam, which he admitted to taking, as well as gabapentin and tramadol. II: A 32-year-old male presented with obtundation and borderline tachycardia after suspected overdose and was intubated for respiratory depression. He was extubated 1 day later, at which time he was subsequently discharged. GC/MS was positive for 3 MEO-PCP and tramadol. III: A 20-year-old male with a history of polysubstance abuse was brought to the emergency department after his family found him to be confused and dystaxic. On arrival to the emergency department, he was tachycardic, somnolent, and dysarthric, and in possession of a bag marked as containing 3-MEO-PCP, which he admitted to taking. A basic drug screen was positive for marijuana and cocaine only. His toxicity resolved the next day.

Discussion: Three patients with exposure to 3-MEO-PCP are presented. While the clinical presentations in each patient may have been confounded by the presence of other substances (which varied), somnolence and an elevated heart rate were present in each case. Each patient survived to hospital discharge without long-term sequelae.

Conclusion: 3-MEO-PCP is an emerging drug of abuse with sedative and dissociative toxicity profiles. 
90. Isopropyl Alcohol Intoxication Following Ingestion of Multiple Alcohol Swabs in the Inpatient Setting

Daria Falkowitz, Joshua Nogar, James Chomin

North Shore University Hopsital, Manhasset, NY, USA

Background: Isopropyl alcohol (IA) has long been abused by alcoholdependent individuals as an inexpensive alternative to ethanol. Ingestions of IA often cause prolonged periods of intoxication, central nervous system depression, as well as ketosis without acidosis due to its acetone metabolite. We present the first case of IA intoxication following ingestion of isopropyl alcohol swabs.

Case Report: A 32-year-old female who was admitted to an inpatient psychiatric facility for alcohol abuse and depression presented to the emergency department (ED) with altered mental status after being found surrounded by "innumerous" empty alcohol swab wrappers. Her vital signs were Temp $97.5^{\circ} \mathrm{F}, \mathrm{HR} 95 \mathrm{bpm}, \mathrm{BP} 91 / 69 \mathrm{mmHg}$, RR 16 breaths/ min, and oxygen saturation $99 \%$ on room air. On exam, she appeared clinically intoxicated with slurred speech and was unable to provide any useful history. Laboratory analysis showed an osmolar gap of $38 \mathrm{mosm} / \mathrm{kg}$. Serum ethanol was negative; urinalysis was positive for acetone; creatinine and calcium were within normal range. Her initial $\mathrm{pH}$ was 7.33 , but quickly rose to 7.40 approximately $3 \mathrm{~h}$ after intravenous hydration with normal saline and PO challenge. Upon further investigation, it became clear that all hand sanitizer solutions at the psychiatric facility were ethyl alcohol-based. She was hospitalized for $24 \mathrm{~h}$ until her clinical intoxication resolved and was discharged back to the psychiatric facility on hospital day \#2.

Case Discussion: Isopropyl alcohol intoxication is not an uncommon occurrence in the ED. Typically, patients acquire IA in the widely available $70-99 \%$ liquid formulation. In this case, our patient did not have access to another alcohol source that would account for her intoxication, osmolar gap, and laboratory findings. She utilized an item commonly found in any medical facility and presented in a manner consistent with IA intoxication.

Conclusion: Isopropyl alcohol intoxication can be achieved via ingestion of multiple alcohol swabs, even in the inpatient setting. Measures should be taken to limit patient access to this potentially hazardous alcohol source.

\section{Human Toxicity Following an Inhalational Exposure to} Transport ${ }^{\circledR}$ Mikron $^{\text {tm }}$ (Acetamiprid and Bifenthrin) Insecticide

Daria Falkowitz ${ }^{1}$, Joshua Nogar ${ }^{1,2}$, David Lee ${ }^{1}$, James Chomin ${ }^{1}$

${ }^{T}$ North Shore University Hospital, Manhasset, NY, USA

${ }^{2}$ Long Island Jewish Medical Center, New Hyde Park, NY, USA

Background: Transport ${ }^{\circledR}$ Mikron $^{\mathrm{TM}}$ is a novel microemultion insecticide spray comprised of acetamiprid and biefnthrin, a neonicotinoid and a pyrethroid, respectively. No data exists for this product regarding human toxicity. Acetamiprid acts as a selective nicotinic acetylcholine receptor agonist. Bifenthrin exerts its effect as a voltage-gated sodium channelopener. We present a case of human toxicity following a prolonged inhalational exposure to acetamiprid and bifenthrin.

Case Report: A 67-year-old female presents to the emergency department (ED) following a 10-h inhalational exposure to 5\% acetamiprid and $6 \%$ bifenthrin that had been sprayed in her house by an exterminator. She presented with profuse diarrhea, vomiting, urination, and leg weakness. On exam, she had diffuse muscle fasciculations with $4 / 5$ muscle strength in her lower extremities, a disconjugate gaze, and a confused mental status. Laboratory analysis was significant for a sodium of $121 \mathrm{mmol} / \mathrm{L}$, potassium of $3.0 \mathrm{mmol} / \mathrm{L}$, chloride of $86 \mathrm{mmol} / \mathrm{L}, \mathrm{HCO}_{3}$ of $18 \mathrm{mmol} / \mathrm{L}$, and calcium of $7.8 \mathrm{mmol} / \mathrm{L}$. The patient's initial creatinine kinase (CK) was $1648 \mathrm{U} / \mathrm{L}$ and peaked the following day at $2561 \mathrm{U} / \mathrm{L}$. She received $15 \mathrm{mg}$ intravenous diazepam to manage her fasiculations. Her gastrointestinal and electrolyte abnormalities resolved within $36 \mathrm{~h}$, and her neurologic symptoms resolved within $48 \mathrm{~h}$. Ultimately, the patient was discharged home on hospital day 3 . We were unable to obtain xenobiotic levels.

Conclusion: We present the first case report of human toxicity associated with exposure to acetamiprid and bifenthrin. Mechanistically, we attribute the nicotinic symptoms to the acetamiprid component. Management of this toxicity includes IV fluids, electrolyte repletion, and benzodiazepines. Providers should monitor CK levels in patients exhibiting muscle fasciculations and anticipate symptom resolution with supportive care alone.

\section{Employment Survey of Recent Medical Toxicology Fellowship Graduates}

Michael Darracq ${ }^{1}$, Derrick Lung ${ }^{2}$, Stephen Thornton ${ }^{3}$

${ }^{T}$ University of California, San Francisco (UCSF) Fresno Medical Education Program, Fresno, CA, USA

${ }^{2}$ California Poison Control System, San Francisco, CA, USA

${ }^{3}$ University of Kansas Medical Center, Kansas City, KS, USA

Background: There is limited information describing the experience of US-Medical Toxicology (TOX)- fellowship graduates.

Research Question: We sought to describe post-fellowship activities and patterns of compensation.

Methods: Contact information was obtained from the directors of the 26 ACGME-accredited TOX Fellowships for graduates 2011-2015. Graduates were asked to complete an online survey describing prefellowship experience (primary medical specialty, fellowship completion immediately following residency), reasons for fellowship training, board certification completion, nature of and percent of time engaged in TOX activities, nature of compensation for TOX activities, job satisfaction, and satisfaction with fellowship.

Results: Sixty-two out of 122 graduates $(51 \%)$ responded. Primary specialty included Emergency Medicine $(n=56)$, Internal Medicine $(n=2)$, and Family Medicine $(n=2)$. Twelve respondents did not enter fellowship immediately; average delay of 4.42 years post residency (range 112). "General interest in the subject" $(n=60)$; "learn something not taught in residency" $(n=32)$; "felt that it would help with obtaining employment" $(n=27)$; "hoped to get full-time job in geographic area of fellowship" ( $n=13)$; and "didn't know what else to do after residency" $(n=4)$ were reported. Forty-three out of 50 eligible graduates completed certification. TOX activities included research $(n=35)$; inpt consult $(n=47)$; outpt consult $(n=15)$; poison control center $(n=31)$; and education $(n=51)$. Forty-nine respondents were employed by academic institution, 12 by community group, and 3 full-time government service. Completion of fellowship was not a factor in negotiated salary for community physicians. Three graduates reported no current involvement with TOX, 51 reported $\leq 50 \%$ of time, and $8>50 \%$. Forty-eight reported compensation for TOX activities. Common forms were "shift buydown" $(n=22)$; "billed consults" $(n=19)$; and "PCC on-call or perdiem pay" $(n=12)$. Eleven reported some form of stipend and 2 reported receiving hospital-based on-call pay. Job satisfaction was reported as very satisfied $(n=49)$; slightly satisfied $(n=5)$; satisfied $(n=5)$; slightly unsatisfied $(n=2)$; and very unsatisfied $(n=1)$. Fellowship completion satisfaction was reported as very satisfied $(n=52)$; slightly satisfied $(n=1)$, satisfied $(n=8)$, and slightly unsatisfied $(n=1)$.

Conclusion: The majority of graduates are engaged in TOX activities, are affiliated with an academic institution, and receive some form of compensation for TOX activities. Job satisfaction and satisfaction with completion of fellowship are generally positive.

\section{Emergency Medicine Residency Toxicology Education: A Survey Study}

Michael Darracq ${ }^{1}$, Patil Armenian ${ }^{1}$, James Comes ${ }^{1}$, Stephen Thornton ${ }^{2}$ ${ }^{T}$ University of California, San Francisco (UCSF) Fresno Medical Education Program, Fresno, CA, USA

${ }^{2}$ University of Kansas Medical Center, Kansas City, KS, USA 
Background: There is limited information describing US emergency medicine (EM)- resident-toxicology (TOX) education.

Research Question: We sought to determine the nature of education through survey of EM-residency-program directors.

Methods: A survey was sent to directors of the 164 ACGME approved US EM residency programs. Follow-up email was sent 1 and 2 weeks following initial request. Respondents were asked whether rotation in TOX (mandatory or elective) was part of the residency curriculum, duration and nature of rotation, whether the rotation was available locally ( $<1 \mathrm{~h}$ drive from home institution), the number of full-time board-certified/eligible TOX faculty, and the number of TOX lectures that were given to residents each calendar year outside of a rotation.

Results: One hundred seven programs responded (65\%). Seventy-one programs reported mandatory rotation $(66 \%)$ and 22 an available elective (21\%). Durations were $4(n=67,72 \%), 3(n=7,4 \%), 2$ $(n=18,18 \%)$, and 1 week $(n=1,1 \%)$. Lecture-based didactics ( $n=87,94 \%)$; "on-call" for consults $(n=59,63 \%)$; rounds on ED or inpatient patients $(n=65,70 \%)$; call-backs to providers from poison control center (PCC) $(n=60,65 \%)$; answering PCC calls ( $n=32,34 \%)$; self-directed learning ( $n=70,75 \%)$; online lectures or other resources $(n=27,29 \%)$; and case-based presentation or discussion ( $n=22,24 \%)$ were reported components. Nine mandatory rotations (mean $6 \mathrm{~h}$; range $2-12 \mathrm{~h}$ ) and seven available electives (mean $4 \mathrm{~h}$; range 2-5 h) were more than $1 \mathrm{~h}$ drive from home institution. Twenty-one programs reported zero (23\%), 22 reported 1 (24\%), 41 reported $2-5$ (44\%), 7 reported 6-10 full-time faculty $(8 \%)$, and 2 programs reported more than 10 full-time faculty (2\%). Of the 14 responding programs with no available rotation, 11 reported zero (79\%), 1 reported one (7\%), and 2 reported 2 full-time TOX faculty $(14 \%)$. The mean number of TOX lectures in programs with mandatory or elective rotations was 11 (range 0-35) and 11.6 (range $0-20)$ without a rotation.

Conclusion: The majority of responding EM training programs have mandatory or elective medical toxicology rotations of 2-4 weeks. However, almost half of the responding programs report 1 or less fulltime medical toxicology faculty. Further investigation is warranted to determine the impact this discrepancy may have on medical toxicology education to EM residents.

\section{Changing Characteristics of Synthetic Cannabinoid Toxicity: a Retrospective Study of U.S. National Poison Center Data - 2010 to 2015}

Ryan C Daro $^{1}$, Samuel K Cordeiro ${ }^{1}$, Hyunuk Seung ${ }^{2}$, Hong K Kim ${ }^{1,3}$

${ }^{T}$ University of Maryland School of Medicine, Baltimore, MD, USA

${ }^{2}$ University of Maryland School of Pharmacy, Baltimore, MD, USA

${ }^{3}$ Maryland Poison Center, University of Maryland School of Pharmacy, Baltimore, MD, USA

Background: In 2015, a surge in synthetic cannabinoid (SC) exposure was reported in the U.S. Anecdotal reports suggested that the clinical characteristics of SC intoxication in 2015 were different compared to that of earlier experiences. Currently, there is limited epidemiologic data describing the trend of clinical effects and medical outcomes as novel SC compounds have been introduced.

Objectives: To evaluate the characteristics of clinical effects and outcomes from SC exposure in the U.S. from 2010 to 2015.

Methods: A retrospective review of SC exposures reported to U.S. poison centers from January 1, 2010 to December 31, 2015 was performed using data from the National Poison Data System. The primary outcome was the change in cardiovascular and CNS effects related to SC exposure. Secondary outcomes included therapeutic interventions, patient disposition, and clinical outcome. Chi-square test was used to detect differences in frequencies of clinical effects and Bonferroni correction was applied for multiple comparisons.
Results: A total of $23,822 \mathrm{SC}$ cases were identified with peak number of cases in $2011(n=6305)$ and $2015(n=6745)$. Six thousand seven hundred forty-five $(28.3 \%)$ of these occurred in the year 2015 alone representing a $225 \%$ increase from 2014 . Statistically significant $(p<0.0001)$ changes were found in the frequencies of bradycardia ( 0.81 vs. $5.78 \%)$, hypotension ( 1.74 vs. $8.55 \%)$, and tachycardia ( 40.98 vs. $26.89 \%$ ) from 2011 to 2015 . During the same period, coma (1.35 vs. $6.38 \%)$ and drowsiness/lethargy (18.83 vs. $30.48 \%)$ also increased. Clinical effect duration increased, specifically 8-24-h and $1-3$-day categories, by 13.6 and $8.9 \%$, respectively $(p<0.001)$. Major effect increased from 3.06 to $10.59 \%$ and a larger proportion of SC cases were admitted to critical care units ( 9.5 vs. $19.6 \%$ ) between 2011 and 2015.

Discussion: Our results suggest that the spectrum of clinical characteristics of SC exposure changed significantly during the study period. This trend may be associated with the introduction of novel SC compounds and continue to evolve as new SCs are introduced. Increasing incidence of CNS and cardiovascular depression in patients with SC toxicity was identified.

Conclusion: Significant changes in the clinical characteristics and medical outcomes from SC exposure were identified from 2010 to 2015.

95. Clinical Description of the Adverse Event Profile of an 11-YearOld Girl Given a Massive Total Dose of Intravenous Lipid Emulsion

Daniel Corwin ${ }^{1}$, Alexis Topjian ${ }^{1}$, Brenda Banwell ${ }^{1}$, Mark Magnusson ${ }^{1}$, Sandra Sheen ${ }^{2}$, Kevin Osterhoudt ${ }^{1,2}$

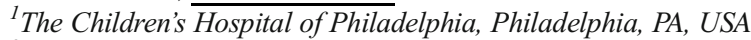

${ }^{2}$ The Poison Control Center at The Children's Hospital of Philadelphia, Philadelphia, PA, USA

Background: Intravenous lipid emulsion (ILE) has been advocated as an antidote for toxicity from intravascular injection of local anesthetic drugs. Goals and endpoints of ILE therapy are still being defined; the range of associated adverse effects, and the toxicity profile, of ILE therapy is still to be characterized. We describe a case of a girl given $66 \mathrm{~mL} / \mathrm{kg}$ of $20 \%$ lipid emulsion in the treatment of presumed mepivacaine toxicity.

Case Report: An 11-year-old girl developed pallor, rolling back of the eyes, and rhythmic muscle twitching after receiving a mandibular nerve block injection with a $1.8-\mathrm{mL}$ ampule of $3 \%$ mepivacaine. Her blood pressure remained stable, but with concern for persistent seizures, she was given three $1 \mathrm{~mL} / \mathrm{kg}$ boluses of ILE, followed by an infusion of $0.25 \mathrm{~mL} / \mathrm{kg} / \mathrm{min}$. The total dose ultimately administered was $3670 \mathrm{~mL}(66 \mathrm{~mL} / \mathrm{kg})$ over $7 \mathrm{~h}$. A serum triglyceride concentration, drawn $2 \mathrm{~h}$ after cessation of ILE infusion, was estimated to be $16,583 \mathrm{mg} / \mathrm{dL}$ after several dilutions; her blood was grossly lipemic. Notable signs included hypersomnolence, tachypnea, and tachycardia. In addition to the hypertriglyceridemia, other complications included apparent metabolic acidosis with hyperlactatemia, difficulty with serum laboratory interpretation, and abnormal contrasting of brain computed tomography. The lipemia cleared over 3 days and the girl recovered; in retrospect, she was not believed to have actually manifested mepivacaine toxicity.

Discussion: Most ILE dosing guidance recommends an upper-bound daily maximum dose of $12.5 \mathrm{~mL} / \mathrm{kg}$. This girl received a very high dose of ILE, equal to an LD-50 derived from an experimental rat model, and approaching total blood volume. Adverse events were associated with the high level of lipemia noted in this case, but full recovery was achieved with supportive care.

Conclusion: This report demontrates the need for cautious use of new therapies, provides data pertaining to the tolerability and adversity of a high dose of ILE, and should inform future development of therapeutic ILE dosing guidelines. 
96. Characteristics of Synthetic Cannabinoid Toxicity by U.S. Geographical Region: A Retrospective Review of National Poison Data System 2010-2015

Samuel K Cordeiro ${ }^{1}$, Ryan C Daro ${ }^{1}$, Hyunuk Seung ${ }^{2}$, Hong K Kim ${ }^{1,3}$ ${ }^{T}$ Department of Emergency Medicine, University of Maryland School of Medicine, Baltimore, MD, USA

${ }^{2}$ Department of Pharmacy Practice and Science, University of Maryland School of Pharmacy, Baltimore, MD, USA

${ }^{3}$ Maryland Poison Center, University of Maryland School of Pharmacy, Baltimore, MD, USA

Background: Synthetic cannabinoids (SC) pose a significant public health problem in the U.S. Each year, novel SC compounds are introduced, outpacing the U.S. government's ability to schedule them. Currently, it is unknown whether there are regional differences in clinical characteristics and medical outcome as novel SC are introduced and sold in different U.S. regions.

Hypothesis: The goal of this study is to evaluate the characteristics of clinical effects and outcomes of SC exposures in different U.S. geographical regions from 2010 to 2015 .

Methods: A retrospective study of SC exposures reported to U.S. poison centers was performed. National Poison Data System (NPDS) data was queried using AAPCC generic code for SC (200617) from January 1, 2010 to December 31, 2015. Regions were identified according to U.S Census definitions. Chi-square test was used to detect difference in clinical effects and Bonferroni correction was applied for multiple comparisons.

Results: In all, 23,822 cases of SC were identified. SC cases increased significantly in the Northeast and South from 2011 to 2015 while decreasing in other regions. Bradycardia, hypotension, coma, and respiratory depression increased in all regions in 2015, notably in Northeast (bradycardia $8.1 \%$ ), West (hypotension $12.1 \%$ ), and Midwest (coma 9.1\%, respiratory depression $7.1 \%)(p<0.0001)$. A larger proportion of SC cases were intubated in 2015, particularly in Midwest $(13.3 \% ; p<0.0001)$ and West $(11.2 \%$; NS). In all regions, except West, the clinical effect duration increased significantly, specifically $8-24$ and $24-72 \mathrm{~h}(p<0.0001)$. All regions showed increases in admission to critical care units, with significant increase in the Midwest $(p<0.0001)$. There was an increase in major effect between 2011 and $2015(p<0.0001)$; the highest net increase occurred in Midwest.

Discussion: Our findings suggest that there are regional differences in clinical effects and outcome from SC toxicity. Increases in bradycardia, hypotension, coma, and respiratory depression were noted with varying frequencies in different regions as well as hospital admission and medical outcome. This finding may be due to the availability of different SC in U.S. regions.

Conclusion: The regional differences in clinical effect and outcome may be correlated with the introduction of novel SC compounds as well as their availability.

\section{Priapism From Low-Dose Clozapine}

James Chomin, Joshua Nogar, Daria Falkowitz

North Shore University Hospital, Manhasset, NY, USA

Background: Clozapine is an atypical antipsychotic that is used to treat refractory schizophrenia. The most feared side effects that have limited its widespread use are agranulocytosis and myocarditis. However, many other side effects still exist, including priapism. Most reported cases of clozapine-associated priapism involve co-exposures to other antipsychotics. We present a case of priapism from exposure to low dose clozapine as a single agent.

Case Report: A 47-year-old male with a past psychiatric history of refractory schizophrenia presented to the ED with $>6 \mathrm{~h}$ of priapism. He had been managed with this single agent for years, but this was his third episode of priapism over the last 3 months; this episode occurred despite a lower dosage than the two prior episodes, and both necessitated cavernosal aspiration to achieve detumescence. His initial ED vital signs were $T=102.5$, BP 147/80, HR 121, RR 18, and $\mathrm{O}_{2}$ Sat $100 \%$. On exam, he was diaphoretic, tachycardic, had a painful erect penis, exhibited lower extremity rigidity, and slight confusion. His labs were significant for a $\mathrm{CK}$ of 2414; urine drug screen from all three ED presentations were negative (including cocaine). During this visit, the patient underwent cavernosal phentolamine injections and aspiration without resolution of his symptoms. He ultimately required a Winter's and Ebbehoj shunt procedure by urology. Although his priapism resolved after the procedure, he remained febrile for $24 \mathrm{~h}$ postoperatively; blood and urine cultures were all negative. His CK, vitals, and mental status returned to normal within $48 \mathrm{~h}$ and he was eventually discharged to psychiatry. His clozapine level upon ED arrival was $255 \mathrm{ng} / \mathrm{mL}$ (reference range 350-600 ng/mL), measured $24 \mathrm{~h}$ after his most recent dose.

Discussion: Many antipsychotics have been reported to cause priapism. The varied incidence of this condition due to typical and atypical agents is thought to be from agent-specific antagonism of the alpha-1 adrenergic receptor. To our knowledge, there are only four prior case reports of priapism due to clozapine exposure as a single agent.

\section{Tramadol and Naloxone: Seizure Association and/or Therapeutic} Effect

Neeraj Chhabra ${ }^{1,2}$, Erin M Pallasch ${ }^{3}$, Michael S Wahl $^{3}$, Sean M Bryant ${ }^{1,2}$ ${ }^{T}$ Toxikon Consortium, Chicago, IL, USA

${ }^{2}$ Cook County (Stroger) Hospital, Chicago, IL, USA

${ }^{3}$ Illinois Poison Center, Chicago, IL, USA

Introduction: Tramadol has been associated with seizures at therapeutic dosing as well as in overdose. Controversy surrounds the efficacy of naloxone in tramadol-intoxication and its association with seizures.

Research Question: We sought to characterize the incidence of tramadol exposures reported to one regional poison center (RPC), the temporal association of seizures with naloxone administration, as well as the response to naloxone.

Methods: RPC cases were retrospectively queried involving tramadol from January 1, 2002 through December 31, 2015. In addition to recording trends for this time period, data abstracted from 2014 and 2015 included clinical details, presence of seizures, administration of naloxone, and response to naloxone.

Results: RPC calls involving tramadol increased steadily from a low of 23 cases in 2002 to 358 cases in 2012. Following a drop in cases to 297 in 2013, the increase continued with 327 cases in 2014 and 415 cases in 2015. Of the 742 cases in 2014 and 2015, 50 (6.7\%) were associated with seizures, all of which were self-limited. Naloxone was administered in 93 $(12.5 \%)$ cases with $9(9.7 \%)$ involving seizures after administration. One case involved a seizure $5 \mathrm{~h}$ post-naloxone administration and another involved a seizure 1 day after discontinuation of a naloxone infusion. Removal of these cases revealed a $7.5 \%$ seizure rate. Of patients receiving naloxone, $23(24.7 \%)$ had a documented beneficial response.

Discussion: Tramadol exposures increased throughout the study period. The only year-to-year drop occurred between 2012 and 2013 which interestingly coincided with the rescheduling of tramadol to a schedule-IV medication in the state served by the RPC. Seizures after tramadol exposure occurred in $6.7 \%$ of cases. Seizures following naloxone administration were also uncommon with some documented many hours after administration. It is unclear whether seizures were related to tramadol, naloxone, unknown coingestants, or preexisting seizure disorder. Naloxone administration had an inconsistent response rate and it is unclear how many intubations were prevented by its administration.

Conclusions: Tramadol exposures reported to this RPC increased markedly during the 14-year study period. The response to naloxone was variable and its association with an increased seizure rate is not supported by these data. 
99. Acetaminophen Poisoning and King's College Criteria: Identifying the Need for a Transfer Guideline

\author{
Neeraj Chhabra ${ }^{1,2}$, Elana N Tan ${ }^{2}$, Carol DesLauriers ${ }^{3}$, Steven E Aks ${ }^{1,2}$, \\ Sean M Bryant ${ }^{1,2}$ \\ ${ }^{1}$ Toxikon Consortium, Chicago, IL, USA \\ ${ }^{2}$ Cook County (Stroger) Hospital, Chicago, IL, USA \\ ${ }^{3}$ Illinois Poison Center, Chicago, IL, USA
}

Introduction: Patients with acetaminophen poisoning and hepatic dysfunction may require inter-hospital transfer to liver transplant centers (LTCs). The cost of transfer has been estimated at more than $\$ 5000$ per patient. Guidelines exist to aid in the decision to transplant livers; however, utilizing these guidelines for transfer decisions may leave patients too sick to benefit from transfer.

Research Question: What are the characteristics of patients transferred to LTCs for acetaminophen poisoning as it relates to the development of King's College Criteria (KCC)?

Methods: Regional poison center (RPC) acetaminophen cases coded for treatment with $\mathrm{N}$-acetyl cysteine (NAC) from January 1, 2012 through December 31, 2015 were retrospectively reviewed. Data abstracted included outcomes, inter-hospital transfers, and laboratory results for KCC (INR $>6.5, \mathrm{pH}<7.30$, creatinine $>3.4 \mathrm{mg} / \mathrm{dL}$ ).

Results: Over the 4-year study period, 285(7.1\%) of 3989 patients were transferred to LTCs. Transfers to pediatric LTCs represented $37.5 \%$ of all transfers, and all survived without transplantation. Of the 178 patients transferred to adult LTCs, 130(73.0\%) never met KCC. Forty patients met KCC prior to transfer. After exclusion of 5 cases for incomplete outcome data, $12(34.3 \%)$ of these patients died and $3(8.6 \%)$ underwent successful liver transplantation. The deaths did not receive transplantation for reasons including death while awaiting transplant (4), brain injury/ death (3), and comorbidities prohibiting transplantation (3). Eight patients met KCC after transfer to a LTC and $2(25 \%)$ died; one too unstable for transplant and another while awaiting transplantation. A subset of 51 patients met KCC but were not transferred to LTCs with 18 (35\%) dying. Of deaths, documented reasons for no transfer included hemodynamic instability (11) and brain injury/death (5).

Discussion: Hospital transfers to LTCs in acetaminophen poisoning comprised $7.1 \%$ of all cases treated with NAC. The majority of patients transferred survived with only NAC, while patients who already met KCC at non-LTCs had a high rate of death even after transfer to LTCs. These patients may have benefitted from earlier transfer.

Conclusion: Further research is needed to develop clinical guidelines to identify patients who would benefit from transfer to LTCs prior to meeting $\mathrm{KCC}$, while avoiding the expensive transfer of patients who would not.

100. Acute Arsenic Toxicity After Oral Ingestion of Intravenous Arsenic Trioxide Purchased on the Internet

Brett Cherrington $^{1}$, Justin Savage ${ }^{3}$, William Eggleston ${ }^{1,2}$
${ }^{1}$ SUNY Upstate Medical University, Department of Emergency Medicine,
Syracuse, NY, USA
${ }^{2}$ Upstate New York Poison Center, Syracuse, NY, USA
${ }^{3}$ University at Buffalo, Department of Emergency Medicine, Buffalo, NY,
USA

Background: Acute arsenic toxicity is uncommon and generally occurs as a result of a suicide or homicide. Toxicity is also reported as a result of therapeutic errors in patients receiving arsenic trioxide for acute promyelocytic leukemia.

Case Report: This is a single patient chart review. A 49-year-old female with a past medical history of hip surgery and chronic pain presented to an emergency department with diarrhea and persistent nausea and vomiting after an intentional ingestion of arsenic trioxide. She reported ingesting 12 vials of $10 \mathrm{mg}$ intravenous arsenic trioxide solution purchased online to "end the pain permanently" $14 \mathrm{~h}$ prior to her presentation. On arrival, she reported odynophagia, headache, and severe abdominal pain. Significant physical exam findings included diaphoresis, pallor, and diffuse lower abdominal tenderness. Her initial vital signs were heart rate, 118/min; blood pressure, 106/62 mmHg; respiratory rate, 18/min; temperature, $38{ }^{\circ} \mathrm{C}$; and $\mathrm{O}_{2}, 95 \%$. Her ECG demonstrated sinus rhythm with a QRS of $62 \mathrm{~ms}$ and a QTc of $435 \mathrm{~ms}$. An abdominal X-ray did not reveal any radio-opaque foreign body.

The patient was treated with a single dose of $3 \mathrm{mg} / \mathrm{kg}$ IM BAL and started on $10 \mathrm{mg} / \mathrm{kg}$ of oral succimer every $8 \mathrm{~h}$ for 7 days followed by $10 \mathrm{mg} / \mathrm{kg}$ of oral succimer every $12 \mathrm{~h}$ for 14 days. A 24 -h urine collection started the day of presentation revealed a total arsenic concentration of $9550 \mathrm{mcg} / \mathrm{L}$ and an inorganic arsenic concentration of $7900 \mathrm{mcg} / \mathrm{L}$. One day after completing the course of chelation, a repeat 24-h urine arsenic level showed a total arsenic concentration of $34 \mathrm{mcg} / \mathrm{L}$ with an undetectable inorganic arsenic concentration. At the time of discharge, the patient did not report any clinical manifestations of peripheral neuropathy.

Discussion: Acute arsenic toxicity causes significant GI symptoms, QTc prolongation, and cardiovascular collapse. Early management involves supportive care, fluid resuscitation, and chelation.

Conclusion: Oral ingestion of intravenous arsenic trioxide solution results in significant absorption and acute toxicity requiring emergent treatment. This product can be purchased online and should be considered in patients with significant GI symptoms and QTc prolongation.

101. Novel Psychoactive Substance Discussions in the Online Webforum Lycaeum Reveal Popular and Unanticipated Drug Combinations

Michael Chary ${ }^{1}$, Alex Manini ${ }^{2}$

${ }^{T}$ United States, United States, USA

${ }^{2}$ United States, United States, USA

Background: Novel psychoactive substances (NPS) are an emerging public health issue, with incompletely understood usage profiles and toxicities. NPS are frequently discussed in web fora and on social media.

Research Question: To provide insight into NPS usage and toxicity, we analyzed unstructured text from a long-standing high-volume online web forum (Lycaeum).

Methods: We conducted a qualitative analysis of substances, substancesubstance combinations, and substance effects mentioned on Lycaeum, an online forum devoted to psychoactive substances. We extracted substances and effects using the Natural Language Toolkit in Python. We processed each post by standardizing spelling and mapping all variants of substance names to one form (lemmatization). To identify patterns in discussion, we clustered posts by substances or effects mentioned. To identify clutters, we used a principal components analysis of the termterm correlation matrix.

Results: We analyzed 107,802 posts on Lycaeum, extracting 806 substances and 58 effects. All posts were in English. The most commonly mentioned substances were LSD and diazepam. The most commonly mentioned classes of substances were hallucinogens and stimulants. The most commonly co-mentioned substances were LSD and MDMA, a recognized combination called "candyflipping." Two patterns emerged. First, posts tended to discuss one of two clusters of effects, "sedativehyponotic and tactile hallucinations" or "stimulant and visual or audio hallucinations". Second, posts discussing mixing psychoactive plants and prescription medications formed two distinct clusters (silhouette coefficient $0.8, p<0.005$, bootstrapping). We also found combinations with no prior discussion in the literature, including guarana and kanna (S. tortuosum) as well as chaliponga (D. cabrerana, a hallucinogenic vine) and M. tenifluora, another hallucinogenic plant.

Discussion: Lycaeum posts centered around creating two types of experiences, combining a relaxed state with tactile hallucinations or combining a stimulated state with audiovisual hallucinations. Posts also described combining psychoactive plants or prescription medications, but not mixing psychoactive plants with prescription medications. Further work is needed to relate online discussion to real-world behaviors. We 
also discovered discussions on novel combinations of psychoactive substances, which, in as much as online discussion reflects behavior in real life, may help focus biochemical and toxicologic work.

\section{Linguistic Differences Between Spanish and English Tweets That Mention Opioids}

Michael Chary ${ }^{1}$, Alex Manini ${ }^{2}$

${ }^{T}$ New York Presbyterian/Queens, Flushing, NY, USA

${ }^{2}$ Elmhurst Hospital Center, Elmhurst, NY, USA

Objective: Social media provides invaluable data for analyzing the nonmedical use of prescription opioids (NMUPO). Drug abuse research to date using social media has focused on English language communications despite the growing importance of Spanish language communications. English and Spanish tweets may discuss different aspects of the nonmedical use of prescription opioids (NMUPO).

Methods: This is a prospective study of publicly available tweets. We included tweets geocoded from the US that contained $>1$ Spanish/English keyword related to NMUPO. We used English keywords, or their Spanish translation, from previous work. We compared these tweets with tweets that contained $>1$ word in English or Spanish, not restricted to NMUPO keywords. We compared Spanish and English tweets on lexical diversity, Flesh-Kincaid grade level, Jacquard similarity, and most common words. Results: We acquired 64,909 tweets geotagged for the US over 1 month that mentioned English or Spanish NMUPD keywords acquired tweets. Spanish and English NMUPD tweets had comparable lexical diversities (0.252 for English, 0.260 Spanish, $p=0.773$, two-tailed $t$ test). General English tweets had significantly greater lexical diversity than general Spanish tweets ( 0.930 for English, 0.468 for Spanish; $p<0.0001$ twotailed $t$ test). General English tweets had significantly higher lexical diversity than NUMPD English tweets ( 0.93 vs $0.252 ; p<0.0001$, twotailed $t$ test), as did Spanish tweets ( 0.468 vs $0.26 ; p<0.0001$, two-tailed $t$ test). Spanish tweets had greater Flesch-Kincaid Reading Ease than English tweets (96.48 vs 63.02 for NUMPD $p<0.0001$; two-tailed $t$ test, 93.18 vs $64.71, p<0.0001$; two-tailed $t$ test). The Flesch-Kincaid Reading Ease of English NMUPD vs general tweets was not significantly different (63.02 vs 6.71; $p=0.88$, two-tailed $t$ test), nor was it for Spanish tweets (93.18 vs 94.68; $p=0.63$, two-tailed $t$ test). The Jaccard similarity between English and English-translated Spanish NMUPD tweets was not significantly different from $0(0.026, p=0.88$; two-sample KolmogorovSmirnov test).

Conclusion: Spanish and English NMUPO tweets use different vocabularies; English tweets use more unique words when discussing NMUPO than other topics than Spanish NMUPO tweets. Spanish NMUPO tweets use fewer words with shorter syllables than English NMUPO tweets.

\section{Do Adolescents Post Social Media Content Indicative of Self-} Harm and Intentional Poisonings?

Brittany Chapman, Jeffrey Lai, Nathalie Nader, Jennifer Carey University of Massachusetts Medical School, Department of Emergency Medicine, Division of Medical Toxicology, Worcester, MA, USA

Background: Suicide is the second leading cause of death among adolescents, with 157,000 teens presenting to the emergency department (ED) annually after a self-harm or suicide attempt. Self-poisoning is the most common method of self-injury among teens evaluated in the ED. Communication behaviors, including those related to self-harm and suicide, may be changing with the near ubiquitous use of technology among adolescents.

Research Question: This study describes adolescent self-harm and suicide attempts via self-poisoning and explores the use of social media among this population in the time prior to their attempts.

Methods: Patients presenting after a self-poisoning between 9 and 22 years old were eligible. After consent, subjects were administered an electronic survey in REDCap regarding their intentions, engagement in social media, recent content posted, and other forms of communication. Electronic medical records were reviewed to gather information on presentation, evaluation, treatment, outcome, and disposition. Additional consent was obtained to review subjects' personal social media accounts. Results: Forty-five subjects consented to the study thus far, and 33 provided access to their personal social media accounts. Thirty subjects reported that they wanted to die, and 23 subjects expected to die. Thirty-six subjects had abnormal vital signs, QTc was prolonged in 9 subjects, and QRS was wide in 2 subjects. Eight subjects received NAC. Twenty-two patients were admitted, 16 to the medical wards 6 to an ICU. The most common social media platforms used by subjects included Facebook, Snapchat, and Instagram. Three subjects reported posting about selfharm; however, when reviewing social media accounts, several additional subjects commented on feeling depressed or wanting to die (data not shown). The top reasons given for not posting about this were embarrassment, being a private person, or not thinking others would care.

Conclusion: Despite increased social media utilization, adolescents are telling others about self-harm attempts in person. However, in contrast to what our study population reported, we discovered references to death, dying, and depression while reviewing posts by subjects who did not claim to have posted this type of content. Further research is being done on social media content posted by suicidal and depressed adolescents

\section{Trends in the Occurrence of Opiate Exposure as Reported to the ToxIC Registry, 2010-2015}

Sharan Campleman ${ }^{1}$, Anne Riederer ${ }^{2}$, Paul Wax ${ }^{1,3}$, Jeff Brent ${ }^{4}$, On Behalf of theToxicology Investigators Consortium (ToxIC)

${ }^{1}$ American College of Medical Toxicology, Phoenix, CA, USA

${ }^{2}$ University of Colorado, School of Medicine, Aurora, CO, USA

${ }^{3}$ University of Texas, Southwestern Medical School, Dallas, TX, USA

${ }^{4}$ University of Colorado, Denver, Aurora, CO, USA

Background: Opioids contribute a major proportion of all agents in the Toxicology Investigators Consortium case registry. In 2015, opioids accounted for nearly $9 \%$ of all agent fields; however, the relative contribution of both the overall opioid class and individual agents has varied over time. The relative class contribution, which includes all natural, semisynthetic, and synthetically derived opioid agonists/antagonists, appears to have declined. Research Question: What changes in toxic events involving individual opioids have occurred over the initial 6 years of reporting to the ToxIC Registry? Methods: This descriptive analysis included all ToxIC Registry cases reported from January 1, 2010 through December 15, 2015. Analysis based cases with at least one agent $(N=37,558,87.1 \%$ cases $)$. Summary statistics included testing of the difference of proportion statistics (prtest) and modeled proportions for trend (ptrend) using "case/total cases" metric (STATA/SE, Statacorp LP).

Results: An average of 915 cases involved exposure to $>1$ opioid annually over the 6 years (range 541-1137), representing $12.5 \%$ to $21.1 \%$ (5year average $14.6 \%$ ). Linear tests for trend showed a consistent downward absolute value (4.2\% AAPC). A positive versus negative trend was observed for single $(+3.3 \%$ AAPC, 3.848 chi-squared $p=0.05)$ versus multiple agent poisonings $(-3.2 \%$ AAPC, $15.95 p<0.0001)$.

Heroin, methadone, oxycodone and tramadol were common in all years (four of top five opioids in single and multiple). Relative rank differences appeared including for heroin $31.6 \%$ single (rank \#1) and $14.6 \%$ of multiple (rank \#3) poisonings. Agent-specific trends varied in direction, magnitude, and significance. In single-agent events, positive trends were observed for buprenorphine, morphine, heroin, and opioid-unspecified, while negative trends appeared for methadone and fentanyl. Multiple agent poisonings had significant increases $(p<0.0001)$ for heroin, hydrocodone, and oxycodone.

Discussion: During this period, cases involving $>1$ opioid demonstrated variation in their relative contribution to the ToxIC registry by specific 
drug and single/multiple exposure. It is important to parse out the relative influence of polydrug exposures, as well as other factors that influence reporting over time.

Conclusion: As the Registry continues to increase in size and accumulated years, the ability to identify stable estimates of trend will continue to improve.

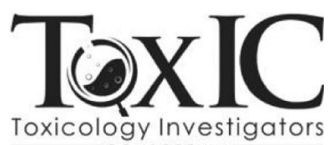

This research was performed in collaboration with the ACMT Toxicology Investigators Consortium.

Toxicology Investigator

105. Barriers and Facilitators to Intensivists' Adherence to Hyperinsulinemia-Euglycaemia Therapy in the Treatment of Calcium Channel Blockers Poisoning

Éric Brassard $^{1}$, Maude St-Onge ${ }^{2}$, Patrick Archambault ${ }^{3}$, Guillaume Lacombe $^{4}$

${ }^{I}$ Département d'anesthésiologie et de soins intensifs, Université Laval, Québec, QC, Canada

${ }^{2}$ Centre antipoison du Québec, CHU de Québec Research Center, Population Health and Optimal Health Practices, Université Laval, Québec, QC, Canada

${ }^{3}$ Centre de recherche du Centre intégré en santé et services sociaux de Chaudière-Appalaches (site Hôtel-Dieu de Lévis, Lévis, QC, Canada

${ }^{4}$ Département de médecine familiale et de médecine d'urgence, Université Laval, Québec, QC, Canada

Background: Calcium channel blocker (CCB) poisoning is an increasing problem associated with significant morbidity and mortality. Hyperinsulinemia-euglycaemia therapy (HIET) has been described in observational studies as being effective and safe. Two studies have shown a low $42 \%$ adherence rate to Poison Control Centers (PCC) recommendations for the management of calcium channel blocker poisonings.

Research Question: To determine the behavioral determinants to adhere to $\mathrm{PCC}$ recommendations about treatment for $\mathrm{CCB}$ poisoning.

Methods: We recruited intensivists by convenience sampling, sending invitations to intensivists across two different provinces in Canada. Eighteen participants from nine different academic hospitals were interviewed. We recruited participants until we reached data saturation. We explored factors influencing the decision to initiate HIET using semistructured interviews and analyzed the content of the interviews with an integrative framework using 14 theoretical domains (Theoretical Domains Framework). Two independent reviewers performed qualitative analysis of the interview transcripts and classified behaviors as being likely to facilitate, likely to be a barrier, or unlikely to affect adherence. We used likely size of the impact and frequency of the behavior to determine relevant domains and resolved disagreements through discussion. Results: We identified the main positive determinants in the following domains: "behavioral regulation" (i.e., algorithm for adjustment of perfusions and information provided to nurses), "self-efficacy" (i.e., confidence about being able to manage HIET), "belief about consequences" (i.e., fear of clinical deterioration or medicolegal consequences), "reinforcement" (i.e., clinical instability), "nature of behavior" (i.e., positive past experiences), and "memory, attention and decision process" (i.e., impact of PCC suggestion to use HIET). We identified the main negative determinants in the following domains: "nature of behavior" (i.e., preference for vasopressors over HIET), "environmental context and resources" (i.e., accessing D50\% and increased nurse workload), and "memory, attention and decision process" (i.e., hypoglycemic or hypokalemic patient, clinical improvement or stability with minimal vasopressor support)
Discussion: Behavioral determinants that we identified might be different in community hospitals. Our results are limited by the explorative nature of this study.

Conclusion: Implementation strategies targeting these behavioral determinants should be developed to improve adherence to $\mathrm{CCB}$ poisoning treatment recommendation.

\section{Covert Drug Use at a Continuation High School}

Nicklaus Brandehoff ${ }^{1}$, Michael Darracq ${ }^{1}$, Jirair Gevorkyan², Robert Torres $^{2}$, Patil Armenian ${ }^{1}$

${ }^{1}$ UCSF-Fresno, Fresno, CA, USA

${ }^{2}$ Central Valley Toxicology, Clovis, CA, USA

Introduction: Clandestine drug use by high school students is a persistent problem and has recently been on the rise, especially on school premises. Many drugs can be dissolved in liquid and inhaled via vaporizers in an attempt to hide drug use from authorities. Furthermore, many of these liquids are flavored, making scent identification less reliable. We aimed to determine if 10 confiscated items from a continuation high school in the Central Valley of California contained illicit drugs.

Methods: Items were confiscated under school policy stating schools are a "drug and alcohol free zone." At the time of confiscation, there was little to no suspicion of illicit drug use. Residue suspected to have active ingredients were isolated from each item and were analyzed in non-targeted fashion by liquid chromatography-time-of-flight-mass spectrometry (LC-TOF/MS) (TOF 6230-LC1200, Agilent) for drugs of abuse. Tetrahydrocannabinol (THC) analysis was performed with tandem liquid chromatography-mass spectrometry (LC-MS/MS, Agilent 6460 QQQ LC/MS).

Results: Ten specimens were analyzed following confiscation. Illegal or suspected illicit drug residues were found in 9/10 items. A cigarette and cigar contained nicotine. White pill "Lilly $3227 / 10 \mathrm{mg}$ " was atomoxetine. A fake lipstick cannister contained methamphetamine and THC. A wrench "socket" device contained nicotine and THC. Zig Zag 1 g nicotine e-liquid bottles "Dragonberry" and "Mango" contained nicotine, pseudoephedrine, and methaqualone. Lush Vapor nicotine e-liquid bottles "Belly Dancer" and "Rescue Me" contained nicotine and oxcarbamazepine.

Conclusion: A variety of prescription and illegal drugs were found in this small sampling of confiscated items at a continuation high school. These drugs were found in containers that had alternative labeling or uses. This observational study demonstrates creativity in obscuring illicit drug use by high school students.

107. Bosak A, Manoj M. Severe inhalational toxicity from chlorine dioxide. Am J Respir Crit Care Med 2016 193;A1612

\section{West Nile Encephalitis: A Mimic of Neuroleptic Malignant} Syndrome

Luke Bisoski $^{1}$, Jodi Mrosko ${ }^{2}$, Andrew King ${ }^{1}$

${ }^{T}$ Wayne State University / Children's Hospital of Michigan Regional Poison Control Center, Detroit, MI, USA

${ }^{2}$ Wayne State University / Detroit Receiving Hospital Emergency Medicine Residency, Detroit, MI, USA

Background: West Nile encephalitis (WNE) is an infectious disease characterized by fever, altered mentation, and muscle weakness or rigidity. Neuroleptic malignant syndrome (NMS) is characterized by hyperthermia, autonomic dysfunction, muscular rigidity, and altered mentation. NMS is most commonly caused by neuroleptic medications.

Hypothesis: West Nile encephalitis can present similarly to NMS.

Methods: A 56-year-old woman with hypertension, diabetes, multiple sclerosis, end-stage renal disease, prior failed pancreas transplant on cyclosporine, and psychiatric disorder on olanzapine presented with a 2-day history of fever, epigastric pain, emesis, and lower extremity weakness. 
During her hospitalization, fevers continued, mental status worsened, and she developed increasing muscular rigidity. Empiric antibiotics and antivirals were started but initial CSF and MRI studies were unremarkable. Bromocriptine therapy was initiated with improvement of fevers and rigidity. Because her mental status did not improve, repeat EEG was performed. This showed seizure activity and prompted further testing.

Results: The patient's exam on hospital day 4 showed and unresponsive woman with occasional grimacing. All four extremities were rigid with cogwheeling in the upper extremities. CPK is 1914. The first CSF sample had one nucleated cell, with negative cultures, and PCR did not detect VZV, HSV 1\&2, herpes 6, CMV, EBV, JC, enteroviruses, and West Nile. Initial CT/and MRI demonstrated chronic changes and EEG was nonspecific. Repeat LP demonstrated 15 nucleated cells and elevated protein. Repeat EEG revealed seizure activity and bitemporal foci of wave discharges. Additional studies including autoimmune, paraneoplastic, and receptor antibody panels and infectious disease testing returned normal. Repeat MRI demonstrated T2 enhancing lesions in the bilateral thalami. West Nile virus serology found elevated IgM antibodies.

Discussion: West Nile virus is a neurotropic flavivirus that causes a spectrum of disease ranging from a mild viral illness to meningitis, encephalitis, acute flaccid paralysis, and death. Involvement of the basal ganglia can cause either a polio-like muscular weakness and paralysis or parkinsonism and rigidity. WNE is diagnosed by viral PCR testing or CSF serology. The improvement of fevers and rigidity with bromocriptine is novel.

Conclusion: WNE should be considered in the differential of NMS. Muscular rigidity may improve with bromocriptine.

\section{A Child with Severe Choreoathetoid Movements Associated with Multiple Psychotropic Medications}

\section{Luke Bisoski, Cynthia Aaron, Andrew King}

Wayne State University / Children's Hospital of Michigan Regional Poison Control Center, Detroit, MI, USA

Background: Psychotropic medications, including stimulants and antipsychotics, are increasingly being used in children for a variety of disorders. Increased use increases the prevalence of adverse drug events (ADEs) and the long-term effects are still largely unknown. Choreoathetoid medication-induced movement disorders are a spectrum of these ADEs. Two causes of choreoathetoid movements in children are tardive dyskinesia (TD) and withdrawal emergent syndrome (WES) and can be difficult to differentiate. TD typically includes orofacial movements and is suspected to be caused by basal ganglia damage. It generally has a worse prognosis while WES is a withdrawal phenomenon and is transient. Diagnosis of both etiologies is clinical.

Hypothesis: Tardive dyskinesia and withdrawal emergent syndrome are possible ADEs caused by psychotropic medications in children and are difficult to differentiate.

Methods: Case Report: A 9-year-old boy with a history of hypertension, hyperactivity, and aggressive/violent behavior presented for agitation, severe choreoathetoid movements, oral dyskinesias, and insomnia. Prescribed medications included risperidone, methylphenidate, labetalol, and clonidine. Recently, his risperidone dose was doubled.

Results: During initial hospitalization, all medications were stopped except for clonidine and labetalol. Benztropine was initiated with subsequent improvement in symptoms. After restarting methylphenidate, he had worsening of choreoathetosis and he was discharged without methylphenidate.

The patient returned the next evening with worsening of choreoathetoid movements and inability to sleep for $30 \mathrm{~h}$. Psychiatry and neurology were consulted and provided contradictory recommendations. Psyhchiatry diagnosed WES and wished to restart risperidone. Neurology diagnosed $\mathrm{TD}$ and recommended starting tetrabenazine, gingko biloba, vitamin E, vitamin B6, and holding risperidone. The patient was transferred to an outside hospital where risperidone was restarted with subsequent improvement of symptoms, and he was discharged home.
Discussion: We present a complex case of choreoathetosis and oral dyskinesias in a young child on multiple psychotropic medications. Medication-related movement disorders can be difficult to differentiate. TD and WES can both initially improve with the escalation of antipsychotic medications. WES is generally benign, but increasing doses of antipsychotic medications can worsen long-term outcomes in TD.

Conclusion: Medical toxicologists should be aware of these two pediatric movement disorders. A definitive diagnosis may remain unclear.

\section{If It Is Not a Toxic Alcohol, What Is It?}

Luke Bisoski $^{1}$, Eric Malone ${ }^{1}$, Michael Smith ${ }^{2}$, Andrew King ${ }^{1}$

${ }^{T}$ Wayne State University / Children's Hospital of Michigan Regional

Poison Control Center, Detroit, MI, USA

${ }^{2}$ William Beaumont Hospital, Royal Oak, MI, USA

Background: Toxic alcohol ingestion is often suspected in patients with an elevated anion gap metabolic acidosis. The corresponding differential is large and toxic alcohols and glycols are often considered, but our clinical experience is that incidence of exposure remains low. Reference texts do not offer an evidence-based ranked differential diagnosis for alternative causes of an elevated anion gap.

Hypothesis: There will be a small number of common alternative diagnosis in patients with an elevated anion gap in which serum ethylene glycol and methanol levels are negative.

Methods: This was a single center retrospective chart review. All ethylene glycol and methanol concentrations sent to a single reference laboratory over a 7-month time period were reviewed. Cases excluded from analysis included those in which the toxic alcohol panel was drawn as part of a nonspecific initial work-up, patients without an elevated anion gap, patients with no data available other than a toxic alcohol panel, and patients with confirmed toxic alcohol ingestions. The final diagnosis for the elevated anion gap was determined by the physician diagnosis in the medical record or interpretation of available data.

Results: One hundred fifty patients were reviewed, and 76 were excluded based on above criteria. Of the 76 excluded cases, a total of four patients $(4 / 76,2.7 \%)$ had a detectable methanol or ethylene glycol level. The most common alternative diagnoses were within four categories. These categories included lactate-associated acidosis $(30 / 74,40.5 \%)$, ketoacidosis $(22 / 74,29.8 \% \%)$, uremia $(14 / 74,18.9 \%)$, and unknown $(8 / 74,10.8 \%)$. Within these categories, more specific differentiation of acidosis included ketoacidosis without hyperglycemia $(15 / 74,20.3 \%)$ and lactateassociated acidosis secondary to seizure $(9 / 74,12.1 \%)$. Additional sources of lactate-associated acidosis included mesenteric ischemia, ibuprofen toxicity, and hepatorenal syndrome.

Discussion: Toxic alcohol ingestion remains a relatively rare cause of an elevated anion gap metabolic acidosis. Physicians should be aware of the most common alterative diagnoses and focus diagnostic testing and management on those etiologies

Conclusion: Lactate-associated acidosis due to seizure and alcoholic ketoacidosis are the most common alternative etiologies in patients with an elevated anion gap acidosis. This data supports the development of an evidence-based ranked differential diagnosis for elevated anion gaps.

\section{Neurocognitive Changes After Exposure to a Single Large Dose of Aluminium Phosphide}

Ashish Bhalla, D Dhananjay, Adarsh Kohli, Surjit Singh, Susheel Kumar, Navneet Sharma

PGIMER, Chandigarh, India

Objective: To determine the neurocognitive function in survivors of acute aluminium phosphide poisoning after stabilisation in the acute phase and at 3 months follow-up. 
DESIGN: Prospective cohort study.

SETTING: Medical Emergency, Department of Internal Medicine, PGIMER Chandigarh, tertiary care centre in North India.

Methods: Twenty-three cases of acute aluminium phosphide poisoning presenting to the emergency/wards/ICU with acute $\mathrm{AlP}_{3}$ poisoning were included in the study. The diagnosis was based on history of ingestion or accidental exposure and the characteristic clinical features. Brain SPECT and perfusion MRI were performed on these patients after stabilisation. Patients having a MMSE of more than 23 were subjected to tests selected for determining the neurocognitive function like trail making test, PGI memory scale, verbal fluency test, Bender visual motor gestalt test.

Results: Test for cognitive functions like Trail making test A: (Test for attention) at baseline was abnormal in 20 patients with the mean time of $115.45( \pm 54.496) \mathrm{s}$, which was significantly more than the normative value with statistically significant improvement $(p<0.000)$ at 3 months with a mean of 95.67 ( \pm 49.058$)$. Trail making test $B$ : (Test for executive function) was abnormal in 16 patients at baseline with a mean time of $139.06( \pm 44.618) \mathrm{s}$, which was significantly more from that of the normative data. With a statistically significant improvement to mean time of $110.91( \pm 27.278) \mathrm{s}(p=0.027)$, Verbal fluency test was significantly lower than that of standard controls. This difference persisted till 3 months. Bender visual motor gestalt test was administered in 22 patients at base line. The mean $( \pm \mathrm{SD})$ errors and DR and at 3 months were significantly more than that of standard control population.

Conclusions: Our study showed that (1) cognitive functions involving the domains of attention, executive function, remote memory, recent memory, mental balance, attention and concentration, delayed recall, verbal new learning, visual retention, semantic memory, and language/ speech and visuospatial functions were impaired in the acute phase of poisoning, when assessed immediately after stabilization and (2) although attention, executive functioning, remote memory, verbal new learning, visual retention, and visuospatial skills improved later at 3 months, there were significant residual defects persisting.

112. Naloxone Administration for Suspected Opioid Overdose in the Pre-Hospital and Emergency Department Settings: A Retrospective Study

Zahra Bakhtiar $^{1}$, Ann-Jeannette Geib ${ }^{1,2}$

${ }^{1}$ Rutgers Graduate School of Biomedical Sciences, Piscataway, NJ, USA

${ }^{2}$ Rutgers Robert Wood Johnson Medical School, Piscataway, NJ, USA

Background: Training and access to naloxone rescue kits to law enforcement officers and emergency medical technicians (EMTs) is promoted in an effort to reduce opioid-related deaths by increasing the number of first responders trained to provide pre-hospital intervention.

Research Question: In this study, we compare the characteristics and clinical outcomes of cases of naloxone administration in the prehospital and ED settings.

Methods: This is a retrospective review of patients treated with naloxone for suspected opioid overdose, in either the pre-hospital or ED setting, at a single center over a 4-year period. A pre-hospital case was defined as a patient receiving an initial naloxone dose in the pre-hospital setting by emergency personnel. An ED case was defined as a patient receiving an initial naloxone dose while in the ED. Comparisons were analyzed with independent $t$ tests for continuous data and chi-squared tests for categorical data.

Results: There were a total of 243 cases of naloxone administration for suspected opioid overdose (173 males, 70 females) with 174 pre-hospital cases and 69 ED cases. Overdose-related morbidity was identified in $51.4 \%$ of total cases, with no significant difference between the two groups. During the period of hospitalization, two patients died in the ED group and no patients died in the pre-hospital group $(p=0.024)$. The relative risk for in-hospital death was 0.08 in pre-hospital cases compared to ED cases, which is not statistically significant $(95 \% \mathrm{CI}=0.004-1.645, p=0.102)$. The cause of death was brain death in one patient, and metastatic colon cancer causing a small bowel obstruction in the other patient.

Discussion: Suspected opioid overdoses initially treated with naloxone in the pre-hospital setting were associated with less in-hospital mortality compared to the ED setting. Furthermore, abnormal physical exam findings and overdose-related morbidities were either less common or no different among pre-hospital cases.

Conclusion: These findings show there are no significant observed adverse effects related to naloxone administration in the pre-hospital setting and may support expansion of naloxone access to emergency personnel to promote early interventions.

113. Inconsistency of Radiopaque Iron Tablets on Serial Abdominal X-Rays After Intentional Iron Overdose

Justin Arnold

University of Alabama at Birmingham, Birmingham, Alabama, USA; Regional Poison Control Center, Children's of Alabama, Birmingham, Alabama, USA

Background: Intentional ingestion of iron-containing tablets are reported to often, but not always, be radiopaque and detectable on abdominal Xray. It is unknown what factors, other than formulation, may influence the detection of iron tablets on abdominal X-ray.

Hypothesis: Iron-containing tablets are inconsistently noted on abdominal X-ray and absence of radiopaque material on abdominal X-ray is a poor predictor for the degree or iron toxicity.

Methods: This is a single patient case report. A healthy 21 -year-old woman presented to the emergency department after an intentional ingestion of "one mouthful" of both ferrous fumarate tablets and household bleach. She vomited within minutes of the ingestion. She complained of nausea, but denied abdominal pain, oral, or throat pain, and was not drooling. Two hours post-ingestion, her serum iron concentration was $200 \mathrm{mcg} / \mathrm{dL}$, WBC was 7.5, and glucose was $91 \mathrm{mg} / \mathrm{dL}$. She had an initial abdominal X-ray that did not demonstrate any radiopaque pill fragments. A second abdominal X-ray was erroneously ordered simultaneously by the resident physician and was obtained 6 min later. The second abdominal X-ray showed a pronounced layering hyperdense material within the gastric body, suspected to be the ingested iron tablets. A repeat serum iron concentration was obtained at $4 \mathrm{~h}$ post-ingestion and had increased to $310 \mathrm{mcg} / \mathrm{dL}$.

Results: Given the presence of radiopaque material on X-ray and increasing serum iron concentration, whole bowel irrigation was performed. The patient only complained of mild nausea and her serum iron concentration decreased to 241 and $92 \mathrm{mcg} / \mathrm{dL}$, at 12 and $16 \mathrm{~h}$ post-ingestion, respectively. Repeat abdominal X-ray $24 \mathrm{~h}$ post-ingestion did not show the previously noted radiopaque material and the patient was discharged to psychiatry.

Discussion: Solid and non-chewable iron preparations are well known to often be radiopaque on abdominal X-rays after acute ingestions. This case report demonstrates that positive X-ray findings after ferrous fumarate ingestion may be a consequence of timing, patient position, or other causes despite the absence of radiopaque material several minutes earlier.

Conclusion: Negative abdominal X-rays after acute iron ingestion do not exclude the presence of a significant iron ingestion.

114. Increased Severity of Neuropsychiatric Outcomes with Acute Synthetic Cannabinoid Toxicity as Compared to Marijuana in Adolescents Presenting to Emergency Departments

Sarah Ann Anderson ${ }^{1}$, Peter Dayan ${ }^{1}$, Diane Calello ${ }^{2}$, Andrew Monte ${ }^{3}$, Anna Oprescu ${ }^{4}$, Yasmin Hurd ${ }^{4}$, Alex Manini ${ }^{4}$, On Behalf of the Toxicology Investigators Consortium (ToxIC)

${ }^{1}$ Columbia University Medical Center, New York, NY, USA 
${ }^{2}$ Robert Wood Johnson University Hospital, New Brunswick, NJ, USA

${ }^{3}$ University of Colorado Denver-Anschutz, Medical Center, Denver, CO, USA

${ }^{4}$ Icahn School of Medicine at Mount Sinai, New York, NY, USA

Background: According to the Substance Abuse and Mental Health Administration, 59\% of emergency department (ED) visits due to synthetic cannabinoid receptor agonists (SCRA) occur among adolescents, ages 12-20. Due to its neurological and psychiatric effects, new data supports that young adults are also more likely to seek treatment for acute SCRA toxicity. Little data exist to provide insight into the neuropsychiatric presentation of SCRA toxicity in adolescents.

Research Question: To characterize the neuropsychiatric presentation of adolescents presenting to the ED following SCRA use as compared to marijuana (MJ) use.

Design/Methods: We conducted a retrospective study on adolescents 10 19 years who presented to the ED for an SCRA or MJ toxicity that was reported to the national Toxicology Investigators Consortium (ToxIC) Registry (2010-15). Included patients had self-reported SCRA and MJ acute exposures and were evaluated by medical toxicology consultation at $>50$ participating registry hospital centers. Relative risk analysis was conducted on the neuropsychiatric symptoms and signs reported in the following clinical subgroups: SCRA-single exposures, SCRA poly drug exposures-excluding MJ, MJ-single exposure, and MJ-poly drug exposures-excluding SCRA.

Results: Adolescents presenting to the ED with single-drug use of SCRA had higher rates of coma/CNS depression (RR 2.48, 95\%CI 1.05-5.96, $p=0.039$ ) and seizures (RR 3.91, 95\%CI 1.18-12.95, $p=0.026$ ) as compared to single-drug MJ users. Any SCRA exposure (single or poly use) is associated with a higher rate of seizures compared to any MJ exposure (see table). Single-drug SCRA exposures had a reduced rate of agitation as compared to single-drug MJ exposures (RR $0.23,95 \% \mathrm{CI}$ $0.14-0.36, p<0.0001)$. However, the opposite is seen in poly-drug users of SCRA compared to poly-drug MJ users with an increased rate of agitation (RR 2.85 95\% CI 1.6255-5.0013 $p=0.0003$ ).

Discussion: This study is the first to analyze the neuropsychiatric outcomes of a large cohort of US adolescents with SCRA toxicity. Limitations include lack of confirmatory testing and failure to capture timing of ingestion.

Conclusions: More severe neuropsychiatric sequellae were associated with SCRA exposures in adolescents as compared to MJ.

\section{This research was performed in collabo- ration with the ACMT Toxicology Investigators Consortium. \\ Toxicology Investigators}

\section{Systematic Review of Adult and Pediatric Lamotrigine Overdose}

Bader Alyahya ${ }^{1,4}$, Marjorie Friesen ${ }^{2}$, Bénédicte Nauche ${ }^{2}$, Martin Laliberté,

${ }^{T}$ Clinical Pharmacology and Toxicology Program, McGill University, Montreal, Canada

${ }^{2}$ McGill University Health Centre, Montreal, Canada

${ }^{3}$ Centre anti-poison du Québec, Quebec City, Canada

${ }^{4}$ King Saud University, Riyadh, Saudi Arabia

Background: Lamotrigine overdose is often described as benign but cases of cardiac arrest and fatalities are reported.

Research Question: What is the range of lamotrigine toxicity in overdose and can serum concentrations predict poisoning severity?

Methods: This is a systematic review of acute lamotrigine overdose. Eight databases including Medline, EMBASE, and the Cochrane Library were searched through October 2015. Only overdose cases with at least one lamotrigine serum concentration were included. Cases of chronic toxicity and those describing side effects of therapeutic use were excluded. Two independent reviewers blinded to the authors' and journals' names selected the records based on eligibility criteria.

Results: We retrieved 5315 records; 39 records (43 cases) met our inclusion criteria. Cases involved primarily adults $(72 \%)$ and potentially lifethreatening features included seizures $(51 \%)$, GCS $\leq 8$ (18\%), hypotension (14\%), and wide-complex tachycardia (WCT) and cardiac arrest (7\%). Of 22 cases of lamotrigine-only exposures (12 adult; 10 pediatric), 2 adult fatalities occurred ( 4 and $7.5 \mathrm{~g}$ ingested) and in $6 / 10$ pediatric cases seizures occurred (all $\leq 2$ years, $83 \%$ with no underlying seizure disorder, minimum $525 \mathrm{mg}$ ingested). The lowest concentration associated with seizures was $3.8 \mathrm{mg} / 1$ (pediatric) and $25.6 \mathrm{mg} / \mathrm{l}$ (adult), suggesting children may be more susceptible to CNS toxicity.

Cardiovascular toxicities occurred almost exclusively in adult patients (threshold $>25 \mathrm{mg} / \mathrm{l}$ ). Interventions included benzodiazepines (58\%), propofol or barbiturates $(9 \%), \mathrm{NaHCO}_{3}(21 \%)$, lipid therapy (14\%), and extracorporeal elimination (12\%). No response to $\mathrm{NaHCO}_{3}$ was reported in 4/9 cases with conduction delays; subsequent lipid therapy achieved response in 2 cases.

Discussion: Lamotrigine is associated with sodium channel blockade. An earlier retrospective review of poison center data for lone lamotrigine overdose suggested relatively benign outcomes; the majority experienced no or minor effects (no deaths). Our review found outcomes consistent with the toxicological profile, namely seizures and cardiac arrest. Our findings are limited by the small number of cases and the inherent publication bias of published case reports.

Conclusion: Lamotrigine concentrations $>25 \mathrm{mg} / \mathrm{l}$ may be predictive of severe toxicity in adults; ingestions as low as $525 \mathrm{mg}$ in those $\leq 2$ years can produce significant CNS toxicity.

\section{Tramadol Exposures Reported to NPDS}

Mohamad Almalki ${ }^{1,2}$, Khalid Almulhim ${ }^{1,2}$, Stephanie Hon ${ }^{1}$, Ziad Kazzi $^{1,2}$, Baian Alabdulbaqi ${ }^{1,2}$

${ }^{1}$ Georgia Poison Center, Atlanta, GA, USA

${ }^{2}$ Emory University, Atlanta, GA, USA

Objectives: To describe tramadol exposures reported to the National Poison Data System by US poison centers over a 10-year period.

Method: This is a retrospective observational study of data collected in the National Poison Data System on a national level from January 2004 to December 2013.This information includes demographics, clinical effects, and outcomes.

Results: There were 47,758 cases documented in National Poison Data System over 10 years (2004-2013). They were distributed into $70 \%$ adults versus $30 \%$ pediatrics $(0-19$ years); $58 \%$ were male. Forty-eight percent were intentional, $45 \%$ were intentional, and $7 \%$ were due to an adverse reaction. $99.5 \%$ of the cases were caused by ingestion. Geographically, Texas constituted the highest percentage with $8.5 \%$ of the cases compared to the lowest of $0.01 \%$ recorded in US Virgin Islands. Forty-two percent of call originated from health care facilities versus $47 \%$ from home. Ninety-four percent of the clinical effect involved CNS. Nine percent developed a seizure, $1 \%$ were intubated, $0.1 \%$ had arrhythmias, and $4 \%$ received naloxone. The exposures resulted in no effect in $23 \%$ of the cases, $20 \%$ experienced minor effects, $15 \%$ moderate effects, and $2.8 \%$ resulted in death.

Discussion: This study is limited by the potential errors associated with cases reported to poison centers in general.

Conclusion: While $95 \%$ of tramadol exposures developed CNS clinical effects, only 9\% resulted in seizure and less than $1 \%$ resulted in arrhythmia. Unfortunately, 1337 exposures resulted in fatal outcomes.

117. Botulism or Myasthenia Gravis? Diagnostic and Therapeutic Challenges in Lebanon

Nadia Alhajri ${ }^{1}$, Eva Rajha ${ }^{2}$, Ezaldeen Numur ${ }^{1}$, Kamal Naserddin ${ }^{2}$, Ramzi Alami $^{2}$, Ziad Kazzi ${ }^{1,2}$, Eveline Hitti ${ }^{2}$ 
${ }^{1}$ Emory University, Atlanta, GA, USA

${ }^{2}$ American University of Beirut, Beirut, Lebanon

Background: Endoscopic botulinum toxin-A injection to the gastric antrum is used for weight reduction by delaying gastric emptying. Iatrogenic botulism has been reported but not from this procedure.

Hypothesis: Can a subtoxic dose of Botox unmask myasthenia gravis or cause botulism?

Method: This a single patient chart review. A 60 -year-old female presented with 11 days of worsening dysphagia, weakness, and dyspnea that started 3 days after an endoscopic injection of 300 units of Botox (toxin A) into her gastric antrum. On exam, she had bilateral ptosis, slurred speech, weak neck and upper extremities muscles, with preserved lower externalities muscle power. Negative inspiratory force (NIF) was -23 $(\mathrm{nl}>-60)$. Neither the testing for botulinum nor the antitoxin is available in Lebanon. Because of the possibility of myasthenia, neostigmine was administered. The patient showed improvement of motor power in all extremities and her NIF measurement. EMG and nerve conduction study showed no evidence for neuro or myopathy. Repetitive nerve stimulation was abnormal and most pronounced in the proximal muscles with a $25 \%$ decrement. Serum acetylcholine receptor antibody was undetectable. The patient was started on pyridostigmine and discharged home 2 days after later. After 2 weeks, the patient reported near resolution of her symptoms. Discussion: The clinical presentation is highly suggestive of botulism although the administered dose $(300 \mathrm{u})$ was subtoxic. Although the diagnosis by mouse lethality assay or by mass spectroscopy is not available in Lebanon, the delay in presentation and the small amount administered would have increased the likelihood of negative results by these methods. The improvement after neostigmine points towards myasthenia; however, it is conceivable that it would also occur in botulism. The antitoxin is expensive and not easily available in Lebanon and likely in other countries in the region.

Conclusion: Botulism testing and treatment remain unavailable in most countries. The role of myasthenia gravis in this patient is unclear and may have served as a confounder considering the negative acetylcholine antibody test. Alternatively, botulism toxin may have unmasked a subclinical myasthenia illness. The role of neostigmine in mild cases of botulism may deserve investigation.

\section{Heart Block Following Supratherapeutic Clonidine Ingestion}

Peter Akpunonu $^{1,2}$, Robert Hendrickson ${ }^{2,1}$

${ }^{T}$ Oregon Health and Science Univeristy, Portland, OR, USA

${ }^{2}$ Oregon Poison Center, Portland, OR, USA

Introduction: We present a case of a 15 -year-old female presenting with AV-conduction delay secondary to clonidine overdose. While AV-conduction delay has been reported previously with clonidine overdose, this is the first case with confirmatory detection of clonidine.

Case Description: A 15-year-old female with past medical history of bradycardia and Mobitz I, AV-block presented to the emergency department(ED) with complaint of intentional ingestion with intent to sleep. Patient ingested three tablets of clonidine of unknown strength. These were previously prescribed to her but were discontinued due to previous suicide attempts. She had previously been referred to cardiology for bradycardia following an unreported self-harm attempt with clonidine. On initial examination, she was somnolent and bradycardic with irregular beats. On EKG, she was noted to have 2nd-degree AV-block with HR 35, PR 284, QRS 96, and QT/ QTc 512/391. An EKG repeated $2 \mathrm{~h}$ later continued to reveal 2nddegree AV-block with HR 41, PR 296, QRS 96, and QT/QTc 536/ 443. Approximately $24 \mathrm{~h}$ after her ingestion, she returned to her native rhythm of Mobitz I, AV-block. Urine drug screen was negative for coingestants and comprehensive screen performed by NMS was positive for clonidine only. Clonidine was serum concentration from blood obtained on arrival to the ED was $0.88 \mathrm{ng} / \mathrm{ml}$. The patient was kept inpatient for 2 days and received standard supportive care. She was then transferred to psychiatry for further care.

Discussion: Normal ranges for clonidine are based upon formulation used: immediate-release, oral $0.50-2.0 \mathrm{ng} / \mathrm{mL}, 2 \mathrm{~h}$ after administration; sustained-release, patch $0.20-2.0 \mathrm{ng} / \mathrm{mL}$, at steady state; sustained-release, oral $0.20-0.27 \mathrm{ng} / \mathrm{mL}, 6.8 \pm 3.6 \mathrm{~h}$ after a $0.1-\mathrm{mg}$ single dose in healthy fed adults. While non-fatal and without lasting harm, this case illustrates the potentially disastrous effects that supratherapeutic clonidine use may have on undiagnosed bradyarrythmia. To our knowledge, this is the first case with quantitated clonidine levels and the absence of other cardioactive coingestants. Great care should be taken before starting any patient on clonidine and a thorough evaluation for underlying arrhythmia should be undertaken.

\section{Methylene Blue Utilization: A Poison Center's Experience}

Arvin Akhavan ${ }^{1}$, Matthew Valento ${ }^{1,2}$, Betty Chen ${ }^{1,2}$

${ }^{T}$ University of Washington Division of Emergency Medicine, Seattle, WA, USA

${ }^{2}$ Washington Poison Center, Seattle, WA, USA

Background: Methylene blue (MB) is used primarily in the treatment of acquired methemoglobinemia. Additionally, MB has been used for refractory hypotension in drug overdose and vasoplegic shock, as it inhibits the vasodilatory effects of nitric oxide at vascular smooth muscle.

Methods: We performed a retrospective record review from a poison control center between June 2006 and September 2016, including calls where $\mathrm{MB}$ was recommended and/or administered. Abstracted data included patient's age, exposures, context, therapies, and outcomes.

Results: Forty-two cases met inclusion criteria. Methemoglobinemia was the indication for $\mathrm{MB}$ recommendation in $35(83.3 \%)$ cases and refractory hypotension in $7(16.7 \%)$ cases.

Of 35 cases of methemoglobinemia, the following drugs were implicated: local anesthetics $(n=17,48.6 \%)$, phenazopyridine $(n=5,14.3 \%)$, nitrites $(n=5,14.3 \%)$, dapsone $(n=4,11.4 \%)$, naphthalene $(n=1,2.9 \%)$, and sodium chlorite $(n=1,2.9 \%)$. Causative agents were not identified in 2 cases. Patients with methemoglobinemia ranged from 1 to 87 years of age. Nineteen $(54.3 \%)$ cases were attributed to adverse drug reactions, 3 $(8.6 \%)$ to intentional abuse, and $3(8.6 \%)$ to suicide attempts. Methemoglobin levels were reported in 28 cases; initial levels ranged from 3.7 to $68.5 \%$. MB was administered in 34 cases. Thirty $(88.2 \%)$ of these patients were admitted, and $4(11.8 \%)$ were discharged from the emergency department. Thirty-one $(91.2 \%)$ patients survived. Final outcome was not reported in the remaining patients. Of the 7 cases of refractory shock, 6 were due to intentional polysubstance ingestion. Drugs implicated include amlodipine, diltiazem, carvedilol, ethanol, benzodiazepines, and metformin. MB was administered in 4 cases; 2 survived, 1 died, and the outcome was not reported in $1 . \mathrm{MB}$ was not administered in 2 patients, both of which survived. In one case of refractory shock, MB was recommended for suspected potassium nitrate overdose. The patient died, but no further data was available.

Conclusion: Review of a poison center's data demonstrates that MB is most commonly recommended for methemoglobinemia and occasionally for treatment of refractory hypotension. Poison centers and medical toxicologists may consider MB for treatment of refractory shock following overdose not responsive to standard therapies. Further research should be pursued regarding MB use in refractory shock.

\section{Potassium Ferrocyanide: A Tale of the Blues}

\section{Cynthia Aaron}

Wayne State University / Children's Hospital of Michigan Regional Poison Control Center, Detroit, MI, USA 
Background: Thallium and cesium poisoning are rare events, and when they occur, rapidly obtaining the chelator Prussian Blue (PB) can be lifesaving. However, the process to obtain PB is extremely arduous.

Hypothesis: To provide a more streamlined approach to obtaining a rare antidote.

Methods/Results: We obtained PB to treat a thallium-poisoned patient and established a process by which this could be repeated by other treating physicians or Poison Centers. After discussion with the Minnesota Poison Center, which had previously obtained PB for another patient, we attempted to obtain PB via the same route. However, we experienced significant roadblocks following the same pathway. We learned that $\mathrm{PB}$ is no longer stocked at REAC/TS, there was no hospital supply of PB in Michigan, and that our state's CBRNE stockpile no longer had PB. We then obtained an emergency supply from the CDC. Eventually, we were able to facilitate shipment from McGuff Compounding Pharmacy in California, the only US licensed supplier of PB (Radiogardase ${ }^{\circledR}$ ) manufactured by Heyltex. Obtaining PB from this supplier was further complicated by restricted operating hours. We were able to locate an alternative source of compounded PB in Florida, but unique Michigan regulations related to compounded pharmaceutical products prevented its transport. We have since created an action plan that provides several alternate paths to rapidly obtain $\mathrm{PB}$.

Discussion: Thallium and cesium poisoning are rare events and treatment is made more difficult because the chelator is difficult to obtain. We found unexpected barriers in securing this antidote through normal channels. However, once involved, the CDC was able to rapidly and graciously provide a temporary source of $\mathrm{PB}$ until we could arrange supplies through normal distribution channels. Because of these barriers, and to avoid the potential for future delays in care, we have tried to make this process smoother and more efficient.

Conclusion: Thallium and cesium poisoning are rare and require prompt antidotal therapy with $\mathrm{PB}$, which is itself rare. We have streamlined a previously arduous process so that $\mathrm{PB}$ can be obtained reliably and emergently by other providers.

2017 ACMT Annual Scientific Meeting Abstract Author Index

Aaron, C 19, 20, 21, 22, 23, 33, 80, 109, 120

Adams, A 53

Akhavan, A 119

Akpunonu, P 118

Aks, S 50, 79, 99

Alabdulbaqi, B 116

Alami, R 117

Alhajri, N 117

Allen, A50

Almalki, M 116

Almulhim, K 116

Alyahya, B 115

Amaducci, A 35

Anderson, I 39

Anderson, SA 114

Anwar, M 67

Archambault, P 105

Armenian, P 55, 93, 106

Arnold, J 113

Babu, K 1, 25, 45

Bakhtiar, Z 112

Baltarowich, L 22

Bangh, S 14, 49

Banwell, B 95

Bartnik, J 71

Baum, CR 50

Bentley, S 34

Berman, A 11
Beuhler, M 27, 59, 60

Bhalla, A 10, 111

Bianchi, B 9

Bisoski, L 19, 20, 22, 108, 109, 110

Blonquist, TM 84

Bosak, A 107

Boyer, E 1, 30, 45

Boyle, K 30

Brandehoff, N 106

Brassard, E 105

Brent, J 3, 4, 29, 104

Brooks, D 27, 51

Bryant. SM 98, 99

Burket, G 35

Burkhart, K 72

Buzalko, R 31

Calello, D 47, 81, 114

Callaway, C 37

Campleman, S 3, 4, 29, 104

Cannon, R 35

Cantrel, L 27

Carey, J103

Carlson, L 32

Castelli, R 48

Chai, P 1, 30, 45

Chapman, B 1, 45, 103

Chary, M 101, 102

Chen, B 38, 119

Cherrington, B 100

Cheung, R 5

Chhabra, N 98, 99

Chhabria, B 10

Chomin, J 90, 91, 97

Cole, PD 84

Cole, J 14, 32, 49, 68

Coleman, J 9

Comes, J 93

Conner, K 8

Considine, K 68

Cook, M 35

Cordeiro, SK 94, 96

Corwin, D 95

Crane, P 8

Dhananjay, D 111

Daro, RC 94, 96

Darracq, M 55, 92, 93, 106

Dawood, A 76

Day, R 35

Dayan, P 114

DesLauriers, C 99

Diamond, F 9

Dickenson, C 50

Dolcourt, B 23

D'Orazio, J 76

Dulaney, A 60

Eckmann, D 2

Eggleston, W 78, 100

Enyart, J 35

Falkowitz, D 90, 91, 97

Farkas, A 62, 88, 89

Farrell, N 44, 87

Farrugia, L 12

Fernandez, M 86

Ferrante, D 44

Fields, A 85

Finkelstein, Y 84 
Fong, A16, 40

Friesen, M 115

Friscia, M 1, 45

Gallagher, R 48

Gallagher, D 48

Garcia, E 47

Garlich, F 27, 28

Geib, AJ 112

Gelfand, B 15

George, N 7

Gerona, R 53, 54

Gevorkyan, J 106

Giblin, E 46

Goldlust, E 44

Graeme, K 24

Graves, R 26

Greene, S 4

Greller, H 43

Griswold, M 1, 45

Gugelmann, H 55

Hack, J 7, 44, 87

Haddad, H 39

Harding, S 43

Hardy, K 2

Hatten, B 42, 82

Hendrickson, R 83, 84, 118

Hettinger, AZ 16, 40

Hieger, M 41, 74

Hindman, D 39

Hitti, E 117

Hoffman, R 5, 56

Hon, S 116

Hosey, N 48

Hughes, A 38

Huh, A 37

Hunter, J 48

Hurd, Y 114

Jalloh, Y 34

Jang, D 2, 26

Janssen, J 35

Jarriel, J 74

Judge, B 23

Kamali, M 8

Kan, V 25

Kang, AM 51

Kao, L 36, 77

Katz, K 35

Kazzi, Z 66, 116, 117

Kelly, M 2

Kerns, W 58

Kessler, K 70

Khan, S 34

Kim, HK 94, 96

King, A 19, 20, 21, 33, 79, 108, 109, 110

Kirsch, AM 32

Kirschner, R 31

Kleinschmidt, K 3, 4

Kohli, A 111

Kolecki, P 57

Kopec, K 66

Krotulski, A 1, 45

Kumar, S 10, 111

Lacombe, G 105

Lai, J 30, 103

Laliberté, M 115

Lambert, D 2
Langley, R 60

Langner, T 53, 54

Lapoint, J 28, 65

Lawson, E 48

Leak, C 15

Lee, D 91

Levine 27, 28, 29

Lintner, C 49

Liss, D 75

Logan, B 1, 45

Love, J 26

LoVecchio, F 28

Loveland, T 8

Lugassy, D 43

Lung, D 92

Lydecker, A 25

Lynch, M 63, 73, 88, 89

Macrae, E 48

Magnusson, M 95

Majlesi, N 70

Malashock, H 24

Malone, E 19, 20, 21, 22, 23, 80, 110

Manini, A 5, 34, 56, 101, 102, 114

Maranda, L 25

Marcks, B 18

Marino, R 17

Maskell, K 41

Manoj, M 107

Mazer-Amirshahi, M 15, 16, 40

McNally, G 9

Menke, N 6

Menke, M 6

Meyers, C 34

Miloradovich, J 57, 86

Montague, A 14

Montante, R 8

Monte, A 114

Moore, E 13, 24

Moss, M 82, 83

Mowry, J 36

Mrosko, J 108

Mudan, A 81

Mullins, M 75

Nader, N 103

Naserddin, K 117

Nauche, B 115

Nefcy, A 80

Nelson, L 47, 50

Nguyen, Eric 86

Nguyen, Elizabeth 50

Nickas, M 71

Nikolaides, J 79

Niruntarai, S 78

Nogar, J 69, 90, 91, 97

Nola, J 52

Numur, E 117

Oakley, E 24

Offerman, S 28

Oprescu, A 114

Ortiz, L 77

Osterhoudt, K 52, 57, 86, 95

Otter, J 76

Pacholski, M 52

Padilla-Jones, A 4

Pallasch, EM 98

Partee, Y 75 
Patrick, K 74

Perrone, J 26, 81

Phillips, T 17, 73

Pietrowski, M 57

Pizon, A 6, 17, 18, 27, 37, 89

Plummer, D 32

Polsky, T 52

Presley, B 9

Quan, D 28

Racz, R 72

Rajha, E 117

Regina, A 11, 69, 70, 71

Reif, M 8

Rhyee, S 12

Rianprakaisang, T 12

Riederer, A 104

Rizvanolli, L 79

Robinson, H 68

Rozum, M 79

Ruck, B 47

Ruha, AM 3, 4, 24, 29

Rusyniak, D 77

Sallan, SE 84

Santos, C 66, 67

Sarkaria, R 65

Sauter, D 15

Savage, E 16, 40

Savage, J 100

Schaack, L 64

Schier, J 67

Schimmel, J 7

Schwarz, E 75

Scott, M 48

Seung, H 94, 96

Shafiq, N 10

Shao, S 63

Sharma, N 10, 111

Sheen, $\mathrm{S} 95$

Shofer, F 2, 26

Shulman, J 62, 63, 73, 88, 89

Silverman, LB 84

Singh, S 111

Skolnik, A 27

Slanzcka, E 48

Slutzman, J 25

Smith, Karen 31

Smith, Katrina 16, 40

Smith, M 20, 110

Spyres, M 4, 27, 28, 29, 61

Steck, A 64

Stevenson, DS 84

Stevenson, KE 84

St-Onge, M 105

Stripp, M 11, 58, 59, 60

Sud, P 11, 69
Suffoletto, B 18

Sullivan, R 78

Suner, S 7

Surmaitis, R 52, 57

Tan, EN 99

Taub, E 56

Thompson, $\mathrm{H} 81$

Thornton, S 48, 53, 54, 55, 92, 93

Tolliver, S 15

Tominack, R 75

Topjian, A 95

Tormoehlen, L 77

Torres, R 106

Tortora, L 11

Tran, G 16, 40

Trella, J 81, 86

Turner, D 50

Valento, M 38, 119

van Wijk, X 61

Vaughan, E 53

Vearrier, D 57

Vijayanathan, V 84.

Vlahov, D 5.

Vohra, R 28, 29, 66

Vora, P 52

Vrooman, LM 84

Wahl, MS 98

Watts, D 24, 51

Wax, P 3, 4, 29, 50, 104

Weber, J 75

Weiland, J 64, 67

Wiegand, T 8, 85

Willard, E 48

Willenbring, B 49

Williams, K 35

Wills, B 41

Wolk, B 28, 29

AnselmWong, A 66

Wratni, R 8

Yanta, J 62, 89

Zell-Kanter, M 46

Zink, B 44

\section{Compliance with Ethical Standards}

Conflicts of Interest: The ACMT Research committee has no conflicts of interests to disclose and no sources of funding for this manuscript other than those supplied by the separate authors of the individual abstracts.

Sources of Funding: None. 